ESAIM: COCV 22 (2016) 1097-1136

DOI: $10.1051 / \mathrm{cocv} / 2016046$
ESAIM: Control, Optimisation and Calculus of Variations

www.esaim-cocv.org

\title{
UNIFORM OBSERVABILITY ESTIMATES FOR LINEAR WAVES*,**
}

\author{
Camille Laurent ${ }^{1}$ and Matthieu Léautaud ${ }^{2}$
}

\begin{abstract}
In this article, we give a completely constructive proof of the observability/controllability of the wave equation on a compact manifold under optimal geometric conditions. This contrasts with the original proof of Bardos-Lebeau-Rauch [C. Bardos, G. Lebeau and J. Rauch, SIAM J. Control Optim. 30 (1992) 1024-1065], which contains two non-constructive arguments. Our method is based on the Dehman-Lebeau [B. Dehman and G. Lebeau, SIAM J. Control Optim. 48 (2009) 521-550] Egorov approach to treat the high-frequencies, and the optimal unique continuation stability result of the authors [C. Laurent and M. Léautaud. Preprint arXiv:1506.04254 (2015)] for the low-frequencies. As an application, we first give estimates of the blowup of the observability constant when the time tends to the limit geometric control time (for wave equations with possibly lower order terms). Second, we provide (on manifolds with or without boundary) with an explicit dependence of the observability constant with respect to the addition of a bounded potential to the equation.
\end{abstract}

Mathematics Subject Classification. 35L05, 93B07, 93B05.

Received January 6, 2016. Accepted June 7, 2016.

\section{INTRODUCTION}

\subsection{Motivation}

This article is devoted to control and observation issues for the wave equation on a $n$-dimensional compact Riemannian manifold $(M, g)$ with or without boundary $\partial M$. Denoting by $\Delta$ the nonpositive Laplace-Beltrami operator on $M$ and by $L$ the selfadjoint operator $-\Delta$ on $L^{2}(M)$ with Dirichlet boundary conditions, the general controllability problem in time $T>0$ is whether, for each data $\left(u_{0}, u_{1}\right)$ one can find a control function $f$ such that the solution $u$ to

$$
\left\{\begin{array}{l}
\partial_{t}^{2} u+L u=b_{\omega} f \\
\left(u(0), \partial_{t} u(0)\right)=\left(u_{0}, u_{1}\right)
\end{array}\right.
$$

\footnotetext{
Keywords and phrases. Wave equation, observability, controllability, geometric control conditions, uniform estimates.

* The first author is partially supported by the Agence Nationale de la Recherche under grants EMAQS ANR-2011-BS01-017-0 and IPROBLEMS ANR-13-JS01-0006.

** The second author is partially supported by the Agence Nationale de la Recherche under grant GERASIC ANR-13-BS01$0007-01$.

1 CNRS UMR 7598 and UPMC Univ Paris 06, Laboratoire Jacques-Louis Lions, 75005 Paris, France. laurent@ann.jussieu.fr

2 Université Paris Diderot, Institut de Mathématiques de Jussieu-Paris Rive Gauche, UMR 7586, Bâtiment Sophie Germain, 75205 Paris cedex 13, France. leautaud@math.univ-paris-diderot.fr
} 
satisfies $\left(u(T), \partial_{t} u(T)\right)=(0,0)$ (or, equivalently, any given state). In this equation the control $f$ acts on the state $u$ only in the set $\omega:=\left\{b_{\omega} \neq 0\right\}$ where $b_{\omega}$ is, say, a continuous function. A classical functional analysis argument [12] reduces this existence problem to that of finding (for the same time $T>0$ ) a constant $\mathfrak{C}_{o b s}>0$, such that all solutions to

$$
\left\{\begin{array}{l}
\partial_{t}^{2} v+L^{*} v=0 \\
\left(v(0), \partial_{t} v(0)\right)=\left(v_{0}, v_{1}\right)
\end{array}\right.
$$

with $\left(v_{0}, v_{1}\right) \in H^{1}(M) \times L^{2}(M)$ satisfy the so called observability inequality

$$
\mathfrak{C}_{o b s} \int_{0}^{T}\left\|b_{\omega} v(t)\right\|_{H^{1}(M)}^{2} \mathrm{~d} t \geq \mathscr{E}_{1}\left(v_{0}, v_{1}\right)=\frac{1}{2}\left(\left\|v_{0}\right\|_{H^{1}(M)}^{2}+\left\|v_{1}\right\|_{L^{2}(M)}^{2}\right) .
$$

Such an estimate translates that the full energy of the state $v$ (which is preserved through time) may be recovered from the sole observation on the (possibly small) set $\omega$ during the time interval $(0, T)$.

Not only the controllability problem and the observability problem are equivalent, but also, in case (1.3) holds, the constant $\mathfrak{C}_{\text {obs }}^{\frac{1}{2}}$ bounds the norm of the control operator $\left(u_{0}, u_{1}\right) \mapsto f$ mapping to the data to be controlled the associated optimal control function $f$ (in appropriate spaces).

A first natural attempt at proving the energy inequality (1.3) in dimension $n \geq 2$ consists in multiplying (1.2) by $\mathfrak{M} v$, where $\mathfrak{M}$ is an appropriate first order differential operator, and perform integrations by parts. Such "multiplier methods" have been developed in a large number of situations, leading to (1.3) under strong geometric conditions on $(\omega, T)$ (see Refs. [22,28]): basically, in the case where $M$ is an open subset of $\mathbb{R}^{n}$, given a point $x_{0} \in \mathbb{R}^{n}$, it is required that $\omega$ contains a neighborhood of the points $x$ of the boundary where $\left(x-x_{0}\right) \cdot n(x)>0$, $n$ being the outgoing normal to $\partial M$ and that $T>2 \sup _{x \in M}\left|x-x_{0}\right|$ (the multiplier is $\mathfrak{M}=\left(x-x_{0}\right) \cdot \partial_{x}$ ).

Another constructive proof uses global Carleman estimates (see e.g. [3,13], which amount to prove positivity properties for $P_{\psi}^{*} P_{\psi}$, where $P_{\psi}=\mathrm{e}^{\tau \psi}\left(\partial_{t}^{2}+L^{*}\right) \mathrm{e}^{-\tau \psi}$ is a conjugated operator. Here, $\tau$ is a large parameter and $\psi$ an appropriately chosen weight function. Unfortunately, global weights $\psi$ that give rise to positivity of $P_{\psi}^{*} P_{\psi}$ require that $(\omega, T)$ satisfy similar conditions as those coming from multiplier methods.

The advantage of these two direct computational methods is that the proofs are constructive and provide with effective bounds, that are uniform with respect to parameters. However, though very effective, they present an important drawback: they require very strong and inappropriate geometric conditions (see [32] for a geometric discussion on multiplier methods). Indeed, they do not capture the main features of wave propagation, stating roughly that most of the energy should travel along rays of geometric optics.

The complete characterization of $(\omega, T)$ for which the observability inequality $(1.3)$ holds was achieved in $[1,2]$ : observability holds if and only if the Geometric Control Condition (GCC) does: every ray of geometric optics enters $\omega$ in the time interval $(0, T)$ (see also [5] for the "only if" part). The proof of $[1,2]$ is based on a compactness-uniqueness argument and splits into two parts:

(1) Proving a relaxed observability inequality

$$
\mathfrak{C} \int_{0}^{T}\left\|b_{\omega} v(t)\right\|_{H^{1}(M)}^{2} \mathrm{~d} t \geq \mathscr{E}_{1}\left(v_{0}, v_{1}\right)-\mathfrak{C}^{\prime} \mathscr{E}_{0}\left(v_{0}, v_{1}\right),
$$

where $\mathscr{E}_{0}\left(v_{0}, v_{1}\right)=\frac{1}{2}\left(\left\|v_{0}\right\|_{L^{2}(M)}^{2}+\left\|v_{1}\right\|_{H^{-1}(M)}^{2}\right)$ is a weaker energy of the data (or, equivalently, of the state). This estimate translates the high-frequency behavior, and relies on the propagation of singularities for the wave equation (see Cor. 2.6 and Lem. 2.7 for a justification of the terminology "high-frequency"). That $(\omega, T)$ should satisfy GCC is used in this step.

(2) Getting rid of the additional term $\mathscr{E}_{0}\left(v_{0}, v_{1}\right)$ in (1.4). This step relies on unique continuation properties and requires less on $(\omega, T)$. 
Both parts of the proof rely on the understanding of two fondamental properties of the wave equation: (1) propagation of high-frequencies along the rays of geometric optics, and (2) "propagation" of low-frequencies according to the tunnel effect.

In the original proof [1,2], Step (1) relies on a closed graph theorem and the propagation of wave front sets. A variant of this proof, proposed by Lebeau [25], relies instead on a contradiction argument and the propagation of microlocal defect measures of Gérard and Tartar [16,47].

After reductio ad absurdum, Step (2) is then equivalent to proving that any solution of (1.2) vanishing on the set $(0, T) \times \omega$ vanishes everywhere. This step can be performed using a global unique continuation results for waves: the global version of the Holmgren theorem, as proved by John [21] in the analytic setting, or by Tataru, Robbiano-Zuily and Hörmander in $[20,42,48]$ in the general case. At the time $[1,2]$ were written, this general unique continuation theorem for waves (under optimal geometric conditions) was not known in the non-analytic case. Bardos-Lebeau-Rauch managed to bypass this argument by using strongly estimate (1.4) and studying the set of invisible solutions, which then reduces the problem to a classical unique continuation result for eigenfunctions of $L^{*}$.

It is clear from this brief discussion that both steps are highly non constructive, so that the full proof may not seem well-suited for tracking the dependence/robustness of the observability constant $\mathfrak{C}_{\text {obs }}$ with respect to parameters (e.g. w.r.t. the observation time $T$, lower order terms added in the operator $L, \ldots$ ).

The aim of this paper is to provide with a constructive proof under optimal geometric conditions. For this, we explain:

- how to replace Step (2) above by the optimal unique continuation estimates obtained by the authors in [23];

- on a compact manifold without boundary, how to replace step (1) using the analysis of Dehman and Lebeau [10].

We illustrate the interest of this approach by keeping track of some parameters in the analysis. Firstly, we give bounds on the blowup of the observability constant $\mathfrak{C}_{o b s}$ as a function of the observation time $T$ when it goes to the limit control time associated to the open set $\omega$, namely $T \rightarrow T_{G C C}(\omega)^{+}$. Secondly, we provide with an explicit bound of the dependence of the observability constant $\mathfrak{C}_{o b s}$ when adding to the equation a potential, i.e. taking $L=-\Delta+c(x)$ in (1.1)-(1.2).

We also hope that the method we develop here might be used for other purposes (e.g. inverse problems, data assimilation, big data, ... ) where getting uniform estimates might be of importance.

\subsection{Main results}

Before stating our results, let us recall some geometric definitions needed to formulate them (see also Appendix B). For $E \subset M$, we define "the largest distance from $E$ to a point in $M$ " by

$$
\mathcal{L}(M, E):=\sup _{x \in M} \operatorname{dist}(x, E) .
$$

We shall also use the notation

$$
T_{U C}(E)=2 \mathcal{L}(M, E),
$$

which, in case $E$ is open, is the minimal time of unique continuation for the wave equation from the set $E$ (see [21] in the analytic setting or $[20,42,48]$ in the general case). In turn, it also provides the optimal time of approximate controllability from the open set $E$.

Assume for a while that $\partial M=\emptyset$. According to [2,38], given an open set $\omega$ and a time $T>0$, we say $(\omega, T)$ satisfies GCC if

for any $\rho \in S^{*} M$, there exists $t \in(0, T)$ so that $\pi\left(\varphi_{t}(\rho)\right) \in \omega$, 
where, $\varphi_{t}$ is the geodesic flow on $S^{*} M$ and $\pi$ the canonical projection $S^{*} M \rightarrow M$ (see Appendix B). We also say that $\omega$ satisfies GCC if there is a time $T>0$ such that $(\omega, T)$ does. If $\omega$ satisfies GCC, then we may define the minimal control time associated to $\omega$ by

$$
T_{G C C}(\omega)=\inf \{T>0,(\omega, T) \text { satisfies } G C C\} .
$$

We also have

$$
\begin{aligned}
T_{G C C}(\omega) & =\sup \{\text { length }(\Gamma), \Gamma \text { geodesic curve on } M \text { with } \Gamma \cap \omega=\emptyset\} \\
& =\inf \{T>0 \text { such that any geodesic curve } \Gamma \text { with } \operatorname{length}(\Gamma) \geq T \text { satisfies } \Gamma \cap \omega \neq \emptyset\} .
\end{aligned}
$$

It can be proved that $T_{G C C}(\omega) \geq T_{U C}(\omega)$ (a proof is given in Appendix B Lemma B.4). Given a continuous function $b_{\omega}$, we also define the constant

$$
\mathfrak{K}(T)=\min _{\rho \in S^{*} M} \int_{0}^{T} b_{\omega}^{2} \circ \pi \circ \varphi_{t}(\rho) \mathrm{d} t,
$$

which is the smallest average of the function $b_{\omega}^{2}$ along geodesics of length $T$. With this definition, we also have $T_{G C C}(\omega)=\inf \{T>0, \mathfrak{K}(T)>0\}=\sup \{T>0, \mathfrak{K}(T)=0\}$, with $\omega:=\left\{b_{\omega} \neq 0\right\}$.

In the case $\partial M \neq \emptyset$, one may also define a (continuous) "broken" geodesic flow on the appropriate phase space (see [2]), and the above definitions still allow to express that $(\omega, T)$ satisfies GCC. When considering the boundary observation/control problem, we need the following definition [2]: given $\Gamma \subset \partial M$ and $T>0$, we say that $(\Gamma, T)$ satisfy the Geometric Control Condition $\mathrm{GCC}_{\partial}$ if every generalized geodesic (i.e. ray of geometric optics) traveling at speed one in $M$ meets $\Gamma$ on a non-diffractive point in a time $t \in(0, T)$.

As already mentioned, we present here two different types of results: first, we estimate $\mathfrak{C}_{o b s}$ as a function of time $T$ when $T \rightarrow T_{G C C}^{+}(\omega)$, and second, we estimate $\mathfrak{C}_{o b s}$ as a function of the potential $c(x)$, when taking $L=-\Delta+c(x)$ in $(1.1)$ and (1.2).

Our first results concern, in the case $\partial M=\emptyset$, the behaviour of the constant $\mathfrak{C}_{o b s}(T)$ as a function of the observation time $T$ when the latter is close to $T_{G C C}(\omega)$. We first prove that the observability estimate (1.3) always fails for the critical time $T=T_{G C C}(\omega)$, and give an explicit blowup rate when $T \rightarrow T_{G C C}(\omega)^{+}$. In all what follows, we assume that $b_{\omega} \in \mathscr{C}^{\infty}(M)$ (or, at least $\mathscr{C}^{k}(M)$ for some large $k$ ).

Theorem 1.1. Assume that $\partial M=\emptyset$ and (1.3) holds for all solutions of (1.2) with $L=-\Delta+1$. Then, $\mathfrak{K}(T)>0$ (i.e. $(\omega, T)$ satisfies $G C C)$ and we have $\mathfrak{C}_{\text {obs }}(T) \geq \mathfrak{K}(T)^{-1}$, where $\mathfrak{K}(T)$ is defined in (1.8).

That is to say that the observability constant $\mathfrak{C}_{\text {obs }}(T)$ blows up at least like $\mathfrak{K}(T)^{-1}$ as $T \rightarrow T_{G C C}(\omega)^{+}$.

We also obtain an upper bound on this blowup rate. Namely, we shall prove the following uniform observability estimate.

Theorem 1.2 (Uniform observation theorem). Assume that $\partial M=\emptyset, \omega=\left\{b_{\omega} \neq 0\right\}$ satisfies $G C C$ and that $T_{U C}(\omega)<T_{G C C}(\omega)$. Then, for any $T_{1}>T_{G C C}(\omega)$, there exist $C, \kappa>0$ such that for any $T \in\left(T_{G C C}(\omega), T_{1}\right]$, any $\left(v_{0}, v_{1}\right) \in H^{1}(M) \times L^{2}(M)$ and $v$ associated solution of (1.2) with $L=-\Delta+1$, we have

$$
\mathscr{E}_{1}\left(v_{0}, v_{1}\right) \leq C \mathrm{e}^{\kappa \mathfrak{K}(T)^{-1}} \int_{0}^{T}\left\|b_{\omega} v(t)\right\|_{H^{1}(M)}^{2} \mathrm{~d} t
$$

where $\mathfrak{K}(T)$ is defined in (1.8).

Summing up Theorems 1.1 and 1.2, we have proved that, for $T>T_{G C C}(\omega)$, we have

$$
\mathfrak{K}(T)^{-1} \leq \mathfrak{C}_{o b s}(T) \leq C \mathrm{e}^{\kappa \mathfrak{K}(T)^{-1}},
$$


where the upper bound requires the assumption $T_{U C}(\omega)<T_{G C C}(\omega)$. Note also that, as an intermediate step of the proof of Theorem 1.2, we obtain the following high frequency estimate:

$$
\int_{0}^{T}\left\|b_{\omega} v(t)\right\|_{H^{1}(M)}^{2} \mathrm{~d} t \geq \mathfrak{K}(T) \mathscr{E}_{1}\left(v_{0}, v_{1}\right)-C_{0} \mathscr{E}_{1 / 2}\left(v_{0}, v_{1}\right),
$$

uniformly for $T$ bounded, where $2 \mathscr{E}_{1 / 2}\left(v_{0}, v_{1}\right)=\left\|v_{0}\right\|_{H^{1 / 2}}^{2}+\left\|v_{1}\right\|_{H^{-1 / 2}}^{2}$. See Proposition 2.5. This is very close to estimate (1.4) with an explicit constant in front of the energy norm. This, together with Theorem 1.1, stresses the fact that $\mathfrak{K}(T)^{-1}$ is the optimal observability constant for high frequency data. See Corollary 2.6 and Lemma 2.7 for a discussion on this topic.

Using the classical duality argument $[8,12,28]$, we deduce from Theorem 1.2 the following uniform control result.

Corollary 1.3 (Uniform control theorem). Under the assumptions of Theorem 1.2, for any $T_{1}>T_{G C C}(\omega)$, there exist $C, \kappa>0$ such that for any $T \in\left(T_{G C C}(\omega), T_{1}\right]$, any $\left(u_{0}, u_{1}\right) \in L^{2}(M) \times H^{-1}(M)$, there exists $f \in L^{2}\left(0, T ; H^{-1}(M)\right)$ with

$$
\|f\|_{L^{2}\left(0, T ; H^{-1}(M)\right)}^{2} \leq C \mathrm{e}^{\kappa \mathfrak{K}(T)^{-1}} \mathscr{E}_{0}\left(u_{0}, u_{1}\right),
$$

(where $\mathfrak{K}(T)$ is defined in (1.8)) such that the associated solution $u$ of $(1.1)$ satisfies $\left(u(T), \partial_{t} u(T)\right)=(0,0)$.

Note that from this result and a commutator argument (see [10,14]) one may deduce a similar bound on the norm of the control in $L^{2}\left(0, T ; H^{s-1}(M)\right)$ for data in $H^{s}(M) \times H^{s-1}(M)$.

In dimension $n \geq 2$, the condition $T_{U C}(\omega)<T_{G C C}(\omega)$ is not very restrictive (and, in particular, is certainly generic with respect to the set $\omega$ or the metric $g)$. Indeed, we prove in Section B.2 that $T_{U C}(\omega)=T_{G C C}(\omega)$ implies a very specific geometric situation. Roughly speaking, it shows that close to the points where the maximum of $\operatorname{dist}(x, \omega)$ is reached, $M \backslash \omega$ is a closed geodesic ball of radius $T_{U C}(\omega) / 2$. A precise statement is given in Lemma B.6.

The estimation of the cost of fast control has already been investigated in several situations: in finite dimension [45], in different situations for the Schrödinger equation [30,34,35], for the heat equation [30, 33], for the Stokes equation $[6]$.

In all these cases, the equations under study are controllable in any time $T>0$ and the question is about to estimating how the observability constant blows up as $T \rightarrow 0^{+}$. We are not aware of any such results in the case of the wave equation in dimension $n \geq 2$, for which a minimal time is required to have observability. The case $n=1$ (to which Thm. 1.2 above does not apply) has been recently investigated in [17]. The authors give an explicit expression of the observability constant with respect to several parameters. In turn, they provide with upper bounds of the cost as a power of $T-T_{G C C}$ (which, in this context, is closely related to $\mathfrak{K}(T)$ ). This power seems different from -1 , but rather -6 (using [17], Thm. 2 with $\gamma=\left(T-T_{G C C}\right) / 8$ and $\eta=\left(T-T_{G C C}\right) / 32$ ).

Note that short time estimates of the control cost for the heat equation are also known to imply uniform estimates of the control for a transport-diffusion equation in the vanishing viscosity limit, see [29]. This problem was originally studied by Coron and Guerrero in [7]. In this context, a minimal time also appears to obtain uniform observability. The question of getting uniform observability in the natural time related to the transport equation remains open.

The above results are particularly simple to write and prove in the case of the Klein-Gordon equation $L=-\Delta+1$. However, they generalize (with some technicalities, but no additional conceptual difficulty) to wave equations with lower order terms. In that context, we wish to consider the control problem (1.1) in case the operator $L$ is a general time-dependent perturbation of $-\Delta$, defined by

$$
L u(t, x)=-\Delta u(t, x)+b_{0}(t, x) \partial_{t} u(t, x)+\left\langle\mathrm{d} u(t, x), b_{1}(t, x)\right\rangle_{x}+c(t, x) u(t, x),
$$

where $b_{0}$ and $c$ are smooth functions on $\mathbb{R} \times M$ and $b_{1}$ is a smooth time dependent vector field on $M$. Note that we may equivalently rewrite $\left\langle\mathrm{d} u(x), b_{1}(x)\right\rangle_{x}=g_{x}\left(\nabla u(x), b_{1}(x)\right)$ (see Appendix B for notations). 
The adjoint observation problem is (1.2) with

$$
L^{*} v(t, x)=-\Delta v(t, x)-\overline{b_{0}}(t, x) \partial_{t} v(t, x)-\left\langle\mathrm{d} v(t, x), \overline{b_{1}}(t, x)\right\rangle_{x}+\left(\bar{c}-\partial_{t} \overline{b_{0}}-\operatorname{div}\left(\overline{b_{1}}\right)\right) v(t, x),
$$

and if $u$ is a smooth solution to (1.1)-(1.9) and $v$ a smooth solution to (1.2)-(1.10), we have the duality identity

$$
\left[\left(\partial_{t} u, v\right)_{L^{2}(M)}-\left(u, \partial_{t} v\right)_{L^{2}(M)}+\left(b_{0} u, v\right)_{L^{2}(M)}\right]_{0}^{T}=\int_{0}^{T}\left(f, b_{\omega} v\right)_{L^{2}(M)} \mathrm{d} t .
$$

In this general setting, we obtain the same results as in the case $L=L^{*}=-\Delta+1$, with an analyticity assumption with respect to time on the coefficients, and where $\mathfrak{K}(T)$ has to be appropriately modified.

Theorem 1.4. Assume that the coefficients of $b_{0}, c$ and $b_{1}$ are smooth and depend analytically on the variable $t$. Then, the analogues of Theorems 1.1 and 1.2 hold for equation (1.2) with $L^{*}$ given by (1.10) and

$$
\mathfrak{K}(T)=\min _{\rho \in S^{*} M} g_{T}^{+}(\rho),
$$

where, denoting by $(x(s), \xi(s))=\varphi_{s}\left(x_{0}, \xi_{0}\right)$, we have

$$
g_{T}^{+}\left(x_{0}, \xi_{0}\right)=\int_{0}^{T} b_{\omega}^{2}(x(t)) \exp \left(\int_{0}^{t} \operatorname{Re}\left(b_{0}\right)(\tau, x(\tau))+\left\langle\frac{\xi(\tau)}{|\xi(\tau)|_{x(\tau)}}, \operatorname{Re}\left(b_{1}\right)(\tau, x(\tau))\right\rangle_{x(\tau)} \mathrm{d} \tau\right) \mathrm{d} t .
$$

In fact, the analogue of Theorem 1.1 (lower bound) does not require the analyticity in time of the coefficients. Analyticity in time (on the time interval $\left[0, T_{1}\right]$, where $T_{1}$ is given in the statement of Thm. 1.2) is however strongly used in the proof of the analogue of Theorem 1.2 (upper bound) which relies on the unique continuation argument of $[20,23,42,48]$. Note that this result would be the same if we replaced the observation equation $\partial_{t}^{2} v+L^{*} v=0$ by $\partial_{t}^{2} v+L v=0$. Indeed, the symbol $g_{T}^{+}(x, \xi)$ (and thus the constant $\mathfrak{K}(T)$ ) only depends on $\operatorname{Re}\left(b_{0}\right)$ and $\operatorname{Re}\left(b_{1}\right)$. Remark that the damped wave equation corresponds to the case $c=0, b_{1}=0$ and $b_{0}$ real valued (see also Rem. 2.13 below).

Let us now consider the problem of obtaining a uniform observability constant $\mathfrak{C}_{\text {obs }}$ for perturbations of $-\Delta$ by a potential $c \in L^{\infty}(M)$. Here, we no longer assume that $M$ has no boundary, and our result work for boundary observation as well. In this setting, Dehman-Ervedoza [9] proved that the constant $\mathfrak{C}_{o b s}$ remains uniformly bounded for $\|c\|_{L^{\infty}}$ bounded. The purpose of the following results is to establish an explicit bound. We have a rough result for general potentials, and a refined one in case $c \in L_{\delta}^{\infty}(M)$, where

$$
L_{\delta}^{\infty}(M)=\left\{c \in L^{\infty}(M ; \mathbb{R}), \delta\|u\|_{L^{2}(M)}^{2} \leq \int_{M}|\nabla u|^{2}+c|u|^{2}, \text { for all } u \in H_{0}^{1}(M)\right\}, \quad \text { for } \delta \geq 0 .
$$

Remark that functions in $L_{\delta}^{\infty}(M)$ are real-valued. In case $\partial M=\emptyset, H_{0}^{1}(M)$ stands for $H^{1}(M)$.

Theorem 1.5. Assume that $(\omega, T)$ satisfies $G C C$, resp. that $(\Gamma, T)$ satisfies $G C C_{\partial}$. Then, for any $c \in L^{\infty}(M)$, any $V_{0}=\left(v_{0}, v_{1}\right) \in H_{0}^{1}(M) \times L^{2}(M)$, and associated solution $v$ of

$$
\left\{\begin{array}{l}
\partial_{t}^{2} v-\Delta v+c(x) v=0, \\
\left.v\right|_{\partial M}=0, \quad \text { if } \partial M \neq \emptyset \\
\left(v(0), \partial_{t} v(0)\right)=\left(v_{0}, v_{1}\right),
\end{array}\right.
$$

we have the estimates

$$
\mathfrak{C}_{o b s} \int_{0}^{T}\|v(t)\|_{H^{1}(\omega)}^{2} \mathrm{~d} t \geq \mathscr{E}_{1}\left(V_{0}\right),
$$


resp.,

$$
\mathfrak{C}_{o b s} \int_{0}^{T}\left\|\partial_{\nu} v(t)\right\|_{L^{2}(\Gamma)}^{2} \mathrm{~d} t \geq \mathscr{E}_{1}\left(V_{0}\right)
$$

with $\mathfrak{C}_{\text {obs }}=\mathfrak{C}_{\text {obs }}\left(\|c\|_{L^{\infty}}\right)$ where $\mathfrak{C}_{\text {obs }}(r)=C \exp (\exp (C \sqrt{r}))$.

If $c \in L_{\delta}^{\infty}(M), \delta>0$, then $\mathfrak{C}_{o b s}=\mathfrak{C}_{o b s}\left(\delta,\|c\|_{L^{\infty}}\right)$ with $\mathfrak{C}_{o b s}(\delta, r)=C \exp \left(C\left(1+\delta^{-1 / 2}\right) r\right)$ (where the constant $C>0$ does not depend on $\delta, r)$.

Even the refined estimate in the case $c \in L_{\delta}^{\infty}(M)$ does not reach the general conjecture of Duyckaerts-ZhangZuazua [13], being that $\mathfrak{C}_{\text {obs }}(r)$ should be of the form $C \exp \left(C r^{\frac{2}{3}}\right)$ for all $c \in L^{\infty}(M)$ in dimension $n \geq 2$. However, whereas the $C \exp \left(C r^{\frac{2}{3}}\right)$ bound is proved (even for time dependent potentials $\left.c \in L^{\infty}((0, T) \times M)\right)$ in [13] in case $(\omega, T)$ satisfy a mutliplier-type condition, to our knowledge, Theorem 1.5 is the first explicit bound under the sole GCC condition. We also refer to [53] for the dependence w.r.t. potentials in dimension one.

As can be seen in the proof, the loss with respect to the expected exponent is probably due to the rough energy estimates we perform and the use of the high and low-frequency results as black boxes.

Note finally that the uniform observability estimate (without explicit bounds) for potentials lying in bounded set of $L^{p}(M), p>d$ is proved in [14]. A modification of the rough argument in the general case should probably allow to give an explicit bound for potentials $c \in L^{d}(M)$, for the unique continuation estimate of [23] also holds for such potentials (using the rough Sobolev estimate $\|c u\|_{L^{2}} \leq\|c\|_{L^{d}}\|u\|_{H^{1}}$ in the proofs of that reference).

\subsection{Idea of the proof and plan of the article}

All proofs of the present paper rely on the optimal quantitative unique continuation results proved by the authors in [23]. To explain the spirit of the proof, let us formulate a typical instance of this result (see [23], Thm. 1.1) in the case $\partial M=\emptyset$ and $L=L^{*}=-\Delta+1$ (the case of Dirichlet boundary conditions may also be considered).

Theorem 1.6 (Quantitative unique continuation for waves). For any nonempty open subset $\omega_{0}$ of $M$ and any $T>T_{U C}\left(\omega_{0}\right)$, there exist $C, \kappa, \mu_{0}>0$ such that for any $\left(v_{0}, v_{1}\right) \in H^{1}(M) \times L^{2}(M)$ and associated solution $v \in C^{0}\left(0, T ; H^{1}(M)\right)$ of $(1.2)$, for any $\mu \geq \mu_{0}$, we have

$$
\left\|\left(v_{0}, v_{1}\right)\right\|_{L^{2}(M) \times H^{-1}(M)} \leq C \mathrm{e}^{\kappa \mu}\|v\|_{L^{2}\left(0, T ; H^{1}\left(\omega_{0}\right)\right)}+\frac{1}{\mu}\left\|\left(v_{0}, v_{1}\right)\right\|_{H^{1}(M) \times L^{2}(M)} .
$$

In the analytic setting, this result is a global quantitative version of the Holmgren theorem and can be proved with the theory developed by Lebeau in [24]. In the $C^{\infty}$ case, the qualitative unique continuation result in optimal time was proved by Tataru [48] (see also $[20,42,50]$ for more general operators). This followed a series of papers: $[26,37]$ in infinite time, and then $[19,39]$. Concerning quantitative results, Robbiano [40] first proved inequality (1.6) for $T$ sufficiently large and $C \mathrm{e}^{\kappa \mu}$ replaced by $C \mathrm{e}^{\kappa \mu^{2}}$. This was improved by Phung [36] to $C_{\varepsilon} \mathrm{e}^{\kappa \mu^{1+\varepsilon}}$, still in large time. In [49], Tataru suggested a strategy to obtain $C_{\varepsilon} \mathrm{e}^{\kappa \mu^{1+\varepsilon}}$ in optimal time (in domains without boundaries). At the same time we proved the above Theorem 1.6 (in a more general framework than the above statement, see [23], Thm. 1.1), Bosi, Kurylev and Lassas [4] obtained $C_{\varepsilon} \mathrm{e}^{\kappa \mu^{1+\varepsilon}}$, still in domains without boundaries (but with constants uniform with respect to the operators involved, for applications to inverse problems). We refer to the introduction of [23] for a more detailed discussion on this issue. One of the motivations for Theorem 1.6 is that it provides the cost of approximate controls for waves (see $[23,40]$ ).

One of the advantages of this result is that it is proved via Carleman estimates and hence furnishes computable constants. In particular, a uniform version with respect to lower order terms is also furnished in [23], which we shall use for the proof of Theorem 1.5. 
With this in hand, the starting point of this paper is a proof of the full observability estimate (1.3) from the high-frequency one (1.4) and (1.16). This is the following very basic observation: plugging (1.16) in (1.4) yields, for all $\mu \geq \mu_{0}$,

$$
\left(1-\frac{2 \mathfrak{C}^{\prime}}{\mu^{2}}\right) \mathscr{E}_{1}\left(v_{0}, v_{1}\right) \leq \mathfrak{C} \int_{0}^{T}\left\|b_{\omega} v(t)\right\|_{H^{1}(M)}^{2} \mathrm{~d} t+2 \mathfrak{C}^{\prime} C^{2} \mathrm{e}^{2 \kappa \mu} \int_{0}^{T}\|v(t)\|_{H^{1}\left(\omega_{0}\right)}^{2} \mathrm{~d} t .
$$

Taking also $\mu \geq \sqrt{2 \mathfrak{C}^{\prime}}$, this eventually proves (1.3) with $\mathfrak{C}_{\text {obs }} \simeq \mathfrak{C}+\mathfrak{C}^{\prime} C^{2} \mathrm{e}^{2 \kappa \sqrt{2 \mathfrak{C}^{\prime}}}$, provided that $\overline{\omega_{0}} \subset \omega$ and $T_{U C}\left(\omega_{0}\right) \leq T_{G C C}(\omega)$ (which we may always assume, see Appendix B.2). This directly provides a quantitative treatment of Step (2): passing from the relaxed observability inequality (1.4) to the full observability inequality (1.3).

On a compact manifold without boundary, we also explain how to prove (1.4) in a constructive way. This follows the spirit of the paper by Dehman and Lebeau [10]. We write the observation as

$$
\int_{0}^{T}\left\|b_{\omega} v(t)\right\|_{H^{1}(M)}^{2} \mathrm{~d} t=\left(\mathscr{G}_{T} V_{0}, V_{0}\right), \quad V_{0}=\left(v_{0}, v_{1}\right),
$$

where $\mathscr{G}_{T}$ is the Gramian operator of the control problem. As in [10], we prove essentially that $\mathscr{G}_{T}$ is a pseudodifferential operator of order zero with principal symbol $\sigma_{0}\left(\mathscr{G}_{T}\right)=\int_{0}^{T} b_{\omega}^{2} \circ \pi \circ \varphi_{t}(\rho) \mathrm{d} t$. We have $\sigma_{0}\left(\mathscr{G}_{T}\right) \geq \mathfrak{K}(T)$ uniformly on $S^{*} M$; the use of the Sharp Gårding inequality then proves that $\left(\mathscr{G}_{T} V_{0}, V_{0}\right) \geq \mathfrak{K}(T) \mathscr{E}_{1}\left(V_{0}\right)$, modulo lower order terms $C \mathscr{E}_{0}\left(V_{0}\right)$, which (if $\mathfrak{K}(T)>0$ ) is exactly (1.4) with $\mathfrak{C}=\frac{1}{\mathfrak{K}(T)}$ and $\mathfrak{C}^{\prime}=\frac{C}{\mathfrak{K}(T)}$.

The plan of the paper is the following. Section 2 is devoted to the study of the limit $T \rightarrow T_{G C C}(\omega)^{+}$. In Section 2.1, we introduce some notation used throughout the paper. Then, in Section 2.2 we perform the highfrequency analysis of a model case, namely the Klein-Gordon equation, corresponding to $L=L^{*}=-\Delta+1$ (and prove in particular Thm. 1.1). In this case, the proofs are simpler to write, so we chose to expose it separately. Then, we conclude in this case the proof of Theorem 1.2 in Section 2.3. Finally, we consider the general case of Theorem 1.4 in Section 2.4. Only the high-frequency analysis needs care, for the low-frequency analysis is exactly that of Section 2.3 .

Then, in Section 3, we consider the problem of uniform observation with respect to potentials. We first prove the refined low-frequency estimates in this case in Section 3.1. Second, we conclude the proof of Theorem 1.5 in Section 3.2, using as a black box the high-frequency estimates of $[1,2]$.

The article ends with two appendices. Appendix A concerns general facts on pseudodifferential calculus. It contains in particular a proof of a non-autonomous non-selfadjoint Egorov theorem (Appendix A.2), of some smoothing properties of operators (Appendix A.3) and some uniform calculus estimates on compact manifolds (Appendix A.4). The second Appendix B is devoted to geometry and contains some elementary properties of $T_{G C C}(\omega)$ and $T_{U C}(\omega)$ (Appendix B.2).

\section{The observability COnstant as $T \rightarrow T_{G C C}(\omega)^{+}$}

In all this section, $\partial M=\emptyset$. In Sections 2.2 and 2.3, we first prove Theorems 1.1 and 1.2: in these two sections, the operator $L$ is $-\Delta+1$. In Section 2.4, we then prove their generalization, namely Theorem 1.4: in that section, $L$ has the general form given in (1.10). The reason why the analysis is simpler in the Klein-Gordon case is that we have the exact factorization formula, for $\Lambda=(-\Delta+1)^{\frac{1}{2}}$,

$$
\partial_{t}^{2}-\Delta+1=\partial_{t}^{2}+\Lambda^{2}=\left(\partial_{t}-i \Lambda\right)\left(\partial_{t}+i \Lambda\right) .
$$

Of course, this formula is not needed (as shown in Sect. 2.4) but it gives rise to several simplifications. We refer to Remark 2.11 concerning the use of an exact square root of $-\Delta+1$. 


\subsection{Preliminaries}

We denote by $\left(e_{j}\right)_{j \in \mathbb{N}}$ a Hilbert basis of eigenfunctions of the Laplace-Beltrami operator, associated to the eigenvalues $\left(\kappa_{j}\right)_{j \in \mathbb{N}}$. In particular, we have $e_{j} \in \mathscr{C}^{\infty}(M),-\Delta e_{j}=\kappa_{j} e_{j}, \kappa_{j} \geq 0$, and $\left(e_{j}, e_{k}\right)_{L^{2}(M)}=\delta_{j k}$.

For $s \in \mathbb{R}$, we shall often use the operator $\Lambda^{s}=(-\Delta+1)^{\frac{s}{2}}: \mathscr{C}^{\infty}(M) \rightarrow \mathscr{C}^{\infty}(M)$, defined spectrally by

$$
\Lambda^{s} f=\sum_{j \in \mathbb{N}}\left(\kappa_{j}+1\right)^{\frac{s}{2}}\left(f, e_{j}\right)_{L^{2}(M)} e_{j}, \quad s \in \mathbb{R}
$$

By duality, it may be extended as an operator $\Lambda^{s}: \mathscr{D}^{\prime}(M) \rightarrow \mathscr{D}^{\prime}(M)$. We define the Sobolev spaces

$$
H^{s}(M)=\left\{f \in \mathscr{D}^{\prime}(M), \Lambda^{s} f \in L^{2}(M)\right\}, \quad s \in \mathbb{R},
$$

and associated inner products

$$
\left.(f, g)_{H^{s}(M)}=\left(\Lambda^{s} f, \Lambda^{s} g\right)_{L^{2}(M)}, \quad\left(\left(f_{1}, f_{2}\right),\left(g_{1}, g_{2}\right)\right)\right)_{H^{s}(M) \times H^{\sigma}(M)}=\left(f_{1}, g_{1}\right)_{H^{s}(M)}+\left(f_{2}, g_{2}\right)_{H^{\sigma}(M)},
$$

and norms

$$
\|f\|_{H^{s}(M)}^{2}=\left\|\Lambda^{s} f\right\|_{L^{2}(M)}^{2}, \quad\left\|\left(f_{1}, f_{2}\right)\right\|_{H^{s}(M) \times H^{\sigma}(M)}^{2}=\left\|f_{1}\right\|_{H^{s}(M)}^{2}+\left\|f_{2}\right\|_{H^{\sigma}(M)}^{2} .
$$

We also sometimes write $H^{s}\left(M ; \mathbb{C}^{2}\right)=H^{s}(M) \times H^{s}(M)$. On any $H^{\sigma}(M), \sigma \in \mathbb{R}$, the operator $\Lambda^{s}$ is an unbounded selfadjoint operator with domain $H^{\sigma+s}(M)$. In particular, $\Lambda^{s}$ is an isomorphism from $H^{\sigma+s}(M)$ onto $H^{\sigma}(M)$.

Let us also recall that, given an open set $\Omega \subset M$, we may define the local $H^{1}$-norm on $\Omega$ by

$$
\|v\|_{H^{1}(\Omega)}^{2}=\int_{\Omega}|\nabla v|^{2}+|v|^{2} \mathrm{~d} x, \quad \text { with }|\nabla v|^{2}(x)=g_{x}(\nabla v(x), \nabla v(x)),
$$

which, in case $\Omega=M$, is equal to the global $H^{1}$-norm defined by (2.2).

We shall also use the energy-spaces $\mathcal{H}^{s}(M)=H^{s}(M) \times H^{s-1}(M)$ associated to the energy norms

$$
\left\|\left(v_{0}, v_{1}\right)\right\|_{\mathcal{H}^{s}(M)}^{2}:=\left\|v_{0}\right\|_{H^{s}}^{2}+\left\|v_{1}\right\|_{H^{s-1}}^{2}, \quad \mathscr{E}_{s}\left(v_{0}, v_{1}\right):=\frac{1}{2}\left\|\left(v_{0}, v_{1}\right)\right\|_{\mathcal{H}^{s}(M)}^{2}
$$

According to [44] (or [46], Thm. 11.2), we have

$$
\Lambda^{s} \in \Psi_{\mathrm{phg}}^{s}(M), \quad \text { with } \quad \sigma_{s}\left(\Lambda^{s}\right)(x, \xi):=\lambda^{s}(x, \xi)=|\xi|_{x}^{s}, \quad(x, \xi) \in T^{*} M \backslash 0,
$$

where all notations are defined in Appendix B. We denote by $\left(\mathrm{e}^{ \pm i t \Lambda}\right)_{t \in \mathbb{R}}$ the group of operators acting on $H^{s}(M)$ generated by $\pm i \Lambda$.

We denote by $\varphi_{t}=\varphi_{t}^{+}$(both notations will be used) the hamiltonian flow of $\lambda(x, \xi)=|\xi|_{x}$ on $T^{*} M \backslash 0$, and $\varphi_{t}^{-}$that of $-\lambda$. They are linked by $\varphi_{t}^{-}=\varphi_{-t}^{+}$, according to Lemma B.2, but is is convenient to keep two different notations.

We conclude this notation section with the following definition.

Definition 2.1. Assume we are given $I=\mathcal{I}_{1} \times \ldots \times \mathcal{I}_{N}$ a product of intervals of $\mathbb{R}$ (possibly reduced to a single interval) and $S$ an application from $I$ with value in the set of bounded linear operators acting from a Banach space $B_{1}$ to another one $B_{2}$. We shall say that $S \in \mathcal{B}\left(I ; \mathcal{L}\left(B_{1} ; B_{2}\right)\right)$ if

(1) there exists $C>0$ such that $\|S(t) u\|_{B_{2}} \leq C\|u\|_{B_{1}}$ for any $u \in B_{1}$ and $t \in I$;

(2) for any $j \in\{1, \ldots, N\}$ and any $\left(t_{1}, \ldots, t_{j-1}, t_{j+1}, \ldots, t_{N}\right) \in \mathcal{I}_{1} \times \ldots \times \mathcal{I}_{j-1} \times \mathcal{I}_{j+1} \times \ldots \times \mathcal{I}_{N}$, the map $t_{j} \rightarrow S\left(t_{1}, \ldots, t_{N}\right) u$ is in $\mathscr{C}^{0}\left(\mathcal{I}_{j} ; B_{2}\right)$ for any $u \in B_{1}$.

Similarly, we write $S \in \mathcal{B}_{\text {loc }}\left(I ; \mathcal{L}\left(B_{1} ; B_{2}\right)\right)$ if this estimate is satisfied on any compact set of $I$. 
In the applications, we always have $I \subset \mathbb{R}$ or $I=\mathcal{I} \times \mathcal{I}$ with $\mathcal{I}$ an interval of $\mathbb{R}$, in particular when studying the solution operator associated to a strictly hyperbolic Cauchy problem, see Appendix A.1.

Note that if $S \in \mathcal{B}\left(I ; \mathcal{L}\left(B_{1} ; B_{2}\right)\right)$ and $T \in \mathcal{B}\left(I ; \mathcal{L}\left(B_{2} ; B_{3}\right)\right)$, then we have $T S \in \mathcal{B}\left(I ; \mathcal{L}\left(B_{1} ; B_{3}\right)\right)$.

Note also that the space $\mathcal{B}\left(I ; \mathcal{L}\left(B_{1} ; B_{2}\right)\right)$ is not included in $L^{\infty}\left(I ; \mathcal{L}\left(B_{1} ; B_{2}\right)\right)$, for maps in $S \in \mathcal{B}\left(I ; \mathcal{L}\left(B_{1} ; B_{2}\right)\right)$ are not a priori measurable in the Bochner sense. However, for all $u \in B_{1}$ and $\left(t_{1}, \ldots, t_{j-1}, t_{j+1}, \ldots, t_{N}\right) \in$ $\mathcal{I}_{1} \times \ldots \times \mathcal{I}_{j-1} \times \mathcal{I}_{j+1} \times \ldots \times \mathcal{I}_{N}$ fixed, the partial map $t_{j} \rightarrow S\left(t_{1}, \ldots, t_{N}\right) u$ is in $\mathscr{C}^{0}\left(\mathcal{I}_{j} ; B_{2}\right)$ and hence (Bochner) integrable. With a usual abuse of notation, for $T_{j} \in \mathcal{I}_{j}$ (and assume $0 \in \mathcal{I}_{j}$ ), we shall write $\int_{0}^{T_{j}} S\left(t_{1}, \ldots, t_{N}\right) \mathrm{d} t_{j}$ the linear map

$$
u \mapsto \int_{0}^{T_{j}}\left(S\left(t_{1}, \ldots, t_{N}\right) u\right) \mathrm{d} t_{j}
$$

Remark then that $\left(t_{1}, \ldots, t_{j-1}, T_{j}, t_{j+1}, \ldots, t_{N}\right) \mapsto \int_{0}^{T_{j}} S\left(t_{1}, \ldots, t_{N}\right) \mathrm{d} t_{j}$ belongs to $\mathcal{B}_{\text {loc }}\left(I ; \mathcal{L}\left(B_{1} ; B_{2}\right)\right)$ if $S$ does. Also, we have $\mathscr{C}^{0}\left(I ; \Psi_{\text {phg }}^{m}(M)\right) \subset \mathcal{B}_{\text {loc }}\left(I ; \mathcal{L}\left(H^{\sigma}(M) ; H^{\sigma-m}(M)\right)\right)$, according to Corollary A.10.

These facts will be used throughout the section.

\subsection{The high-frequency estimate for the Klein-Gordon equation}

In the present case of the Klein-Gordon equation, that is (1.2) with $L^{*}=-\Delta+1$, and in view of the factorization formula (2.1), we use the following splitting:

$$
v_{+}=\frac{1}{2}\left(v_{0}-i \Lambda^{-1} v_{1}\right), \quad v_{-}=\frac{1}{2}\left(v_{0}+i \Lambda^{-1} v_{1}\right),
$$

so that

$$
v_{0}=v_{+}+v_{-}, \quad v_{1}=i \Lambda\left(v_{+}-v_{-}\right) .
$$

we denote by $\Sigma$ the isomorphism corresponding to the splitting (2.3):

$$
\begin{aligned}
\Sigma: H^{s}(M) \times H^{s-1}(M) & \rightarrow H^{s}(M) \times H^{s}(M) \\
\left(v_{0}, v_{1}\right) & \mapsto \quad\left(v_{+}, v_{-}\right) .
\end{aligned}
$$

that is

$$
\Sigma=\frac{1}{2}\left(\begin{array}{cc}
1 & -i \Lambda^{-1} \\
1 & i \Lambda^{-1}
\end{array}\right), \quad \Sigma^{-1}=\left(\begin{array}{cc}
1 & 1 \\
i \Lambda & -i \Lambda
\end{array}\right) .
$$

Notice that the operator $\Sigma$ is (almost) an isometry $H^{s}(M) \times H^{s-1}(M) \rightarrow H^{s}(M) \times H^{s}(M)$. Indeed, if $\left(v_{+}, v_{-}\right)=\Sigma\left(v_{0}, v_{1}\right)$, we have

$$
\left\|\left(v_{0}, v_{1}\right)\right\|_{H^{s}(M) \times H^{s-1}(M)}^{2}=\left\|v_{+}+v_{-}\right\|_{H^{s}(M)}^{2}+\left\|v_{+}-v_{-}\right\|_{H^{s}(M)}^{2}=2\left(\left\|v_{+}\right\|_{H^{s}(M)}^{2}+\left\|v_{-}\right\|_{H^{s}(M)}^{2}\right),
$$

that is

$$
\left\|v_{+}\right\|_{H^{s}(M)}^{2}+\left\|v_{-}\right\|_{H^{s}(M)}^{2}=\mathscr{E}_{s}\left(v_{0}, v_{1}\right)=\mathscr{E}_{s}\left(\Sigma^{-1}\left(v_{+}, v_{-}\right)\right) .
$$

According to (2.1), the expression of the solution of System (1.2) is simply

$$
v(t)=\mathrm{e}^{i t \Lambda} v_{+}+\mathrm{e}^{-i t \Lambda} v_{-} .
$$

We can now recall a result of [10] (in a slightly different context), providing a characterization of the Gramian operator (in the wave splitting (2.3)). 
Proposition 2.2. Denoting by $V_{0}=\left(v_{0}, v_{1}\right) \in H^{s}(M) \times H^{s-1}(M)$ the initial data for System (1.2), we have

$$
\int_{0}^{T}\left\|b_{\omega} v(t)\right\|_{H^{s}(M)}^{2} \mathrm{~d} t=\left(\mathcal{G}_{T} \Sigma V_{0}, \Sigma V_{0}\right)_{H^{s}(M) \times H^{s}(M)},
$$

where

$$
\mathcal{G}_{T}=\int_{0}^{T}\left(\begin{array}{cc}
\mathrm{e}^{-i t \Lambda} B \mathrm{e}^{i t \Lambda} \mathrm{e}^{-i t \Lambda} B \mathrm{e}^{-i t \Lambda} \\
\mathrm{e}^{i t \Lambda} B \mathrm{e}^{i t \Lambda} & \mathrm{e}^{i t \Lambda} B \mathrm{e}^{-i t \Lambda}
\end{array}\right) \mathrm{d} t, \quad B=\Lambda^{-2 s} b_{\omega} \Lambda^{2 s} b_{\omega} .
$$

Moreover, the operator $\mathcal{G}_{T}$ can be decomposed as $\mathcal{G}_{T}=G_{T}+R_{T}$ with

$$
R_{T} \in \mathcal{B}_{\text {loc }}\left(\mathbb{R}^{+} ; \mathcal{L}\left(H^{\sigma}\left(M ; \mathbb{C}^{2}\right) ; H^{\sigma+1}\left(M ; \mathbb{C}^{2}\right)\right), \quad \text { for all } \sigma \in \mathbb{R},\right.
$$

and $G_{T} \in \mathscr{C}^{\infty}\left(\mathbb{R}_{T} ; \Psi_{\mathrm{phg}}^{0}\left(M ; \mathbb{C}^{2 \times 2}\right)\right)$ has principal symbol

$$
\sigma_{0}\left(G_{T}\right)=\left(\begin{array}{cc}
\int_{0}^{T} b_{\omega}^{2} \circ \varphi_{t}^{-} \mathrm{d} t & 0 \\
0 & \int_{0}^{T} b_{\omega}^{2} \circ \varphi_{t}^{+} \mathrm{d} t
\end{array}\right) \in S_{\mathrm{phg}}^{0}\left(T^{*} M, \mathbb{C}^{2 \times 2}\right) .
$$

Note that the Gramian operator $\mathcal{G}_{T}$ actually depends on the space $H^{s}(M)$ (even not written in the notation). An interesting fact is that its principal symbol does not depend on $s$. The result of Proposition 2.2 is essentially proved in ([10], Sect. 4.1) and we reproduce a proof below for the sake of completeness.

Remark 2.3. Note that the operator $B=\Lambda^{-2 s} b_{\omega} \Lambda^{2 s} b_{\omega}$ is symmetric on $H^{s}(M)$ since

$$
(B g, h)_{H^{s}(M)}=\left(\Lambda^{s}\left(\Lambda^{-2 s} b_{\omega} \Lambda^{2 s} b_{\omega}\right) g, \Lambda^{s} h\right)_{L^{2}(M)}=\left(b_{\omega} g, b_{\omega} h\right)_{H^{s}(M)}, \quad g, h \in H^{s}(M) .
$$

Proof of Proposition 2.2. We write $\Sigma V_{0}=\left(v_{+}, v_{-}\right), v(t)=\mathrm{e}^{i t \Lambda} v_{+}+\mathrm{e}^{-i t \Lambda} v_{-}$the associated solution, and develop the inner product

$$
\begin{aligned}
\int_{0}^{T}\left\|b_{\omega} v(t)\right\|_{H^{s}(M)}^{2} \mathrm{~d} t & =\int_{0}^{T}\left(\Lambda^{s} b_{\omega}\left(\mathrm{e}^{i t \Lambda} v_{+}+\mathrm{e}^{-i t \Lambda} v_{-}\right), \Lambda^{s} b_{\omega}\left(\mathrm{e}^{i t \Lambda} v_{+}+\mathrm{e}^{-i t \Lambda} v_{-}\right)\right)_{L^{2}(M)} \mathrm{d} t \\
& =\int_{0}^{T}\left(\Lambda^{-2 s} b_{\omega} \Lambda^{2 s} b_{\omega}\left(\mathrm{e}^{i t \Lambda} v_{+}+\mathrm{e}^{-i t \Lambda} v_{-}\right),\left(\mathrm{e}^{i t \Lambda} v_{+}+\mathrm{e}^{-i t \Lambda} v_{-}\right)\right)_{H^{s}(M)} \mathrm{d} t .
\end{aligned}
$$

This directly yields the sought form for the operator $\mathcal{G}_{T}$ given by (2.9). The Egorov Theorem A.3 (see also Rem. A.5) in the Appendix then implies that

$$
\left(\begin{array}{cc}
\int_{0}^{T} \mathrm{e}^{-i t \Lambda} B \mathrm{e}^{i t \Lambda} \mathrm{d} t & 0 \\
0 & \int_{0}^{T} \mathrm{e}^{i t \Lambda} B \mathrm{e}^{-i t \Lambda} \mathrm{d} t
\end{array}\right)=G_{T}+R_{T}^{0}
$$

with $G_{T} \in \mathscr{C}^{\infty}\left(\mathbb{R}_{T} ; \Psi_{\mathrm{phg}}^{0}\left(M ; \mathbb{C}^{2 \times 2}\right)\right)$ has principal symbol given by $(2.10)$ and $R_{T}^{0} \in$ $\mathcal{B}_{\text {loc }}\left(\mathbb{R}^{+} ; \mathcal{L}\left(H^{\sigma}\left(M ; \mathbb{C}^{2}\right) ; H^{\sigma+1}\left(M ; \mathbb{C}^{2}\right)\right)\right.$ for all $\sigma \in \mathbb{R}$. Finally, Lemma A.6 implies that

$$
R_{T}^{1}=\left(\begin{array}{cc}
0 & \int_{0}^{T} \mathrm{e}^{-i t \Lambda} B \mathrm{e}^{-i t \Lambda} \mathrm{d} t \\
\int_{0}^{T} \mathrm{e}^{i t \Lambda} B \mathrm{e}^{i t \Lambda} \mathrm{d} t & 0
\end{array}\right)
$$

is also in $\mathcal{B}_{\text {loc }}\left(\mathbb{R}^{+} ; \mathcal{L}\left(H^{\sigma}(M) ; H^{\sigma+1}(M)\right)\right.$ for all $\sigma \in \mathbb{R}$, which concludes the proof with $R_{T}=R_{T}^{0}+R_{T}^{1}$.

As a first consequence of Proposition 2.2, we deduce a proof of Theorem 1.1. 
Proof of Theorem 1.1. Let $\rho_{0}=\left(x_{0}, \xi_{0}\right) \in S^{*} M$ that realizes the minimum in (1.8), that is,

$$
\mathfrak{K}(T)=\min _{\rho \in S^{*} M} \int_{0}^{T} b_{\omega}^{2} \circ \pi \circ \varphi_{t}(\rho) \mathrm{d} t=\int_{0}^{T} b_{\omega}^{2} \circ \pi \circ \varphi_{t}\left(\rho_{0}\right) \mathrm{d} t .
$$

Take a local chart $\left(U_{\kappa}, \kappa\right)$ of $M$ such that $x_{0} \in U_{\kappa}$. We denote by $\left(y_{0}, \eta_{0}\right)$ the coordinates of $\rho_{0}$ in this chart. We choose $\psi \in \mathscr{C}_{c}^{\infty}\left(\mathbb{R}^{n}\right)$ such that $\operatorname{supp}(\psi) \subset \kappa\left(U_{\kappa}\right)$, and $\psi=1$ in a neighborhoodof $y_{0}$. Next we define

$$
w^{k}(y)=C_{0} k^{\frac{n}{4}} \mathrm{e}^{i k \varphi(y)} \psi(y), \quad \text { with } \varphi(y)=y \cdot \eta_{0}+i\left(y-y_{0}\right)^{2} \text { and } C_{0}>0 .
$$

Setting now

$$
v_{+}^{k}=\Lambda^{-s} \kappa^{*} w^{k} \in \mathscr{C}_{c}^{\infty}(M),
$$

we have $v_{+}^{k} \rightarrow 0$ in $H^{s}(M), \lim _{k \rightarrow \infty}\left\|v_{+}^{k}\right\|_{H^{s}(M)}=1$ for an appropriate choice of $C_{0}$. Moreover, a classical computation on $\left(w^{k}\right)_{k \in \mathbb{N}}$ shows that $\left(v_{+}^{k}\right)_{k \in \mathbb{N}}$ satisfies

$$
\left(A v_{+}^{k}, v_{+}^{k}\right)_{H^{s}(M)} \rightarrow \sigma_{0}(A)\left(\rho_{0}\right), \quad \text { for all } A \in \Psi_{\mathrm{phg}}^{0}(M) .
$$

Next, we set $v_{-}^{k}=0$ for all $k \in \mathbb{N}$, and $V^{k}=\Sigma^{-1}\left(v_{+}^{k}, v_{-}^{k}\right) \in H^{s}(M) \times H^{s-1}(M)$, so that $\mathscr{E}_{s}\left(V^{k}\right) \rightarrow 1$ as $k \rightarrow \infty$. Applying now (2.8) to $V^{k}$, we have

$$
\int_{0}^{T}\left\|b_{\omega} v^{k}(t)\right\|_{H^{s}(M)}^{2} \mathrm{~d} t=\left(\mathcal{G}_{T} \Sigma V^{k}, \Sigma V^{k}\right)_{H^{s}(M) \times H^{s}(M)},
$$

where $v^{k}(t)$ is the solution to System (1.2) with initial data $V^{k}$. Proposition 2.2 and (2.17) also imply

$$
\begin{aligned}
\lim _{k \rightarrow \infty}\left(\mathcal{G}_{T} \Sigma V^{k}, \Sigma V^{k}\right)_{H^{s}(M) \times H^{s}(M)} & =\lim _{k \rightarrow \infty}\left(\left(G_{T}+R_{T}\right) \Sigma V^{k}, \Sigma V^{k}\right)_{H^{s}(M) \times H^{s}(M)} \\
& =\lim _{k \rightarrow \infty}\left(G_{T} \Sigma V^{k}, \Sigma V^{k}\right)_{H^{s}(M) \times H^{s}(M)} \\
& =\int_{0}^{T} b_{\omega}^{2} \circ \pi \circ \varphi_{t}\left(\rho_{0}\right) \mathrm{d} t=\mathfrak{K}(T),
\end{aligned}
$$

where we used that $R_{T}$ is 1-smoothing, that $G_{T} \in \Psi_{\mathrm{phg}}^{0}(M)$ has principal symbol given by (2.10), and the choice of $\rho_{0}$ in (2.15). Finally using the assumed observability estimate (1.3) with $V^{k}$, and taking the limit $k \rightarrow \infty$ yields

$$
\mathfrak{C}_{o b s}(T) \mathfrak{K}(T) \leftarrow \mathfrak{C}_{o b s}(T) \int_{0}^{T}\left\|b_{\omega} v^{k}(t)\right\|_{H^{s}(M)}^{2} \mathrm{~d} t \geq \mathscr{E}_{s}\left(V^{k}\right) \rightarrow 1 .
$$

This implies $\mathfrak{C}_{\text {obs }}(T) \geq \mathfrak{K}(T)^{-1}$, and concludes the proof of Theorem 1.1.

Remark 2.4. Note that (2.17) translates the fact that the sequence $\left(v_{+}^{k}\right)_{k \in \mathbb{N}}$ is a pure sequence admitting the $H^{s}$-microlocal defect measure $\delta_{\rho=\rho_{0}}$ in the sense of [16,47]. Similarly, the $H^{s}$-microlocal defect measure of the sequence $\left(V^{k}\right)_{k \in \mathbb{N}}$ is

$$
\mu=\left(\begin{array}{cc}
\delta_{\rho_{0}} & 0 \\
0 & 0
\end{array}\right) .
$$

As a second consequence of Proposition 2.2, we also obtain the following high-frequency observability inequality. 
Proposition 2.5. For any $T_{0}>0$, there exists a constant $C_{0}>0$ such that for all $T \in\left[0, T_{0}\right]$, for all $V_{0}=$ $\left(v_{0}, v_{1}\right) \in H^{s}(M) \times H^{s-1}(M)$ and associated solution $v$ of $(1.2)$, we have

$$
\int_{0}^{T}\left\|b_{\omega} v(t)\right\|_{H^{s}(M)}^{2} \mathrm{~d} t \geq \mathfrak{K}(T) \mathscr{E}_{s}\left(V_{0}\right)-C_{0} \mathscr{E}_{s-1 / 2}\left(V_{0}\right)
$$

where $\mathfrak{K}(T)$ is defined by (1.8) and $L^{*}=-\Delta+1$.

Proof of Proposition 2.5. We first write $\Sigma V_{0}=\left(v_{+}, v_{-}\right)=V$, and use (2.8). We have

$$
\left(\mathcal{G}_{T} V, V\right)_{H^{s}\left(M ; \mathbb{C}^{2}\right)}=\left(G_{T} V, V\right)_{H^{s}\left(M ; \mathbb{C}^{2}\right)}+\left(R_{T} V, V\right)_{H^{s}\left(M ; \mathbb{C}^{2}\right)} .
$$

Using that $R_{T} \in \mathcal{B}_{\text {loc }}\left(\mathbb{R}^{+} ; \mathcal{L}\left(H^{s-1 / 2}\left(M ; \mathbb{C}^{2}\right) ; H^{s+1 / 2}\left(M ; \mathbb{C}^{2}\right)\right)\right.$, we have

$$
\left(R_{T} V, V\right)_{H^{s}\left(M ; \mathbb{C}^{2}\right)} \leq\left\|R_{T} V\right\|_{H^{s+1 / 2}\left(M ; \mathbb{C}^{2}\right)}\|V\|_{H^{s-1 / 2}\left(M ; \mathbb{C}^{2}\right)} \leq C_{T}\|V\|_{H^{s-1 / 2}\left(M ; \mathbb{C}^{2}\right)}^{2},
$$

where $C_{T}$ is bounded on compact time intervals.

Next, according to $(2.10)$, the principal symbol of the operator $G_{T}-\mathfrak{K}(T) \operatorname{Id} \in \Psi_{\mathrm{phg}}^{0}\left(M ; \mathbb{C}^{2 \times 2}\right)$ is

$$
\sigma_{0}\left(G_{T}-\mathfrak{K}(T) \mathrm{Id}\right)=\left(\begin{array}{cc}
\int_{0}^{T} b_{\omega}^{2} \circ \varphi_{t}^{-} \mathrm{d} t-\mathfrak{K}(T) & 0 \\
0 & \int_{0}^{T} b_{\omega}^{2} \circ \varphi_{t}^{+} \mathrm{d} t-\mathfrak{K}(T)
\end{array}\right),
$$

which is diagonal with nonnegative components since, according to Corollary B.3, we have

$$
\mathfrak{K}(T)=\min _{\rho \in S^{*} M} \int_{0}^{T} b_{\omega}^{2} \circ \varphi_{t}(\rho) \mathrm{d} t=\min _{\rho \in S^{*} M} \int_{0}^{T} b_{\omega}^{2} \circ \varphi_{t}^{-}(\rho) \mathrm{d} t .
$$

Using the Gårding inequality of Theorem A.9 gives the existence of $C>0$ such that, for all $V \in H^{s}\left(M ; \mathbb{C}^{2}\right)$ all $T \in\left[0, T_{0}\right]$,

$$
\left(\left(G_{T}-\mathfrak{K}(T) \operatorname{Id}\right) V, V\right)_{H^{s}\left(M ; \mathbb{C}^{2}\right)} \geq-C\|V\|_{H^{s-1 / 2}\left(M ; \mathbb{C}^{2}\right)}^{2} .
$$

Combining (2.19), (2.20) and (2.21) now yields the existence of $C>0$ such that, for all $V \in H^{s}\left(M ; \mathbb{C}^{2}\right)$ all $T \in\left[0, T_{0}\right]$,

$$
\left(\mathcal{G}_{T} V, V\right)_{H^{s}(M) \times H^{s}(M)} \geq \mathfrak{K}(T)\|V\|_{H^{s}\left(M ; \mathbb{C}^{2}\right)}^{2}-C\|V\|_{H^{s-1 / 2}\left(M ; \mathbb{C}^{2}\right)}^{2} .
$$

Recalling (2.6) that $\|V\|_{H^{\sigma}\left(M ; \mathbb{C}^{2}\right)}^{2}=\mathscr{E}_{\sigma}\left(V_{0}\right)$ concludes the proof of (2.18).

To conclude this section, we explain the terminology "high-frequency observability estimates". Let first $T_{0}>$ $T_{G C C}(\omega)$ be fixed and denote by $C_{0}>0$ the associated constant given by Proposition 2.5. We define the following $T$-dependent subset of $\mathcal{H}^{s}$ by

$$
\mathcal{H}_{H F}^{s}(T)=\left\{V_{0} \in \mathcal{H}^{s}, \quad \mathscr{E}_{s-\frac{1}{2}}\left(V_{0}\right) \leq \frac{\mathfrak{K}(T)}{4 C_{0}} \mathscr{E}_{s}\left(V_{0}\right)\right\} .
$$

Note that this space is nonlinear. It is however homogeneous in the sense that $V_{0} \in \mathcal{H}_{H F}^{s}(T) \Longrightarrow \mathbb{R} V_{0} \in$ $\mathcal{H}_{H F}^{s}(T)$. Remark also that $\mathcal{H}_{H F}^{s}(T)=\{0\}$ if $T \leq T_{G C C}(\omega)$, since $\mathfrak{K}(T)=0$ in this case. We may now formulate an immediate corollary of Proposition 2.5, only consisting in a rewriting of that statement for data in $\mathcal{H}_{H F}^{s}(T)$, yielding a full observability inequality.

Corollary 2.6. For all $V_{0}=\left(v_{0}, v_{1}\right) \in \mathcal{H}_{H F}^{s}(T)$ and associated solution $v$ of $(1.2)$, we have

$$
\int_{0}^{T}\left\|b_{\omega} v(t)\right\|_{H^{s}(M)}^{2} \mathrm{~d} t \geq \frac{\mathfrak{K}(T)}{2} \mathscr{E}_{s}\left(V_{0}\right) .
$$


Finally, the following Lemma states that data spectrally supported at high-frequency (in terms of the spectral theory of $-\Delta)$ are in $\mathcal{H}_{H F}^{s}(T)$. As such, they satisfy the full observability inequality (2.22).

Lemma 2.7. Denoting by

$$
F_{\kappa}^{s}=\left\{V_{0} \in \mathcal{H}^{s}, \Pi_{\kappa} V_{0}=0\right\}, \quad \text { with } \quad \Pi_{\kappa}\left(v_{0}, v_{1}\right)=\left(\sum_{\kappa_{j} \leq \kappa}\left(v_{0}, e_{j}\right)_{L^{2}(M)} e_{j}, \sum_{\kappa_{j} \leq \kappa}\left(v_{1}, e_{j}\right)_{L^{2}(M)} e_{j}\right),
$$

we have

$$
\kappa \geq\left(\frac{4 C_{0}}{\mathfrak{K}(T)}\right)^{2}-1 \quad \Longrightarrow \quad F_{\kappa}^{s} \subset \mathcal{H}_{H F}^{s}(T) .
$$

When doing this, notice that we compare the typical frequency $\kappa^{\frac{1}{2}}$ to the blow up of the observation $\mathfrak{K}(T)^{-1}$. We recall that $\left(\frac{4 C_{0}}{\mathfrak{\kappa}(T)}\right)^{2} \underset{T \rightarrow T_{G C C}^{+}(\omega)}{\longrightarrow}+\infty$.

Proof. If $(u, v) \in F_{\kappa}^{s}$, then we have $\Pi_{\kappa}(u, v)=0$ so that, with $u_{j}=\left(u, e_{j}\right)_{L^{2}(M)}$ and $v_{j}=\left(v, e_{j}\right)_{L^{2}(M)}$, we obtain

$$
\begin{aligned}
2 \mathscr{E}_{s-\frac{1}{2}}(u, v) & =\sum_{\kappa_{j}>\kappa}\left(\kappa_{j}+1\right)^{s-\frac{1}{2}}\left|u_{j}\right|^{2}+\left(\kappa_{j}+1\right)^{s-\frac{3}{2}}\left|v_{j}\right|^{2} \\
& \leq(\kappa+1)^{-\frac{1}{2}} \sum_{\kappa_{j}>\kappa}\left(\kappa_{j}+1\right)^{s}\left|u_{j}\right|^{2}+\left(\kappa_{j}+1\right)^{s-1}\left|v_{j}\right|^{2} \\
& \leq(\kappa+1)^{-\frac{1}{2}} 2 \mathscr{E}_{s}(u, v) .
\end{aligned}
$$

If now $\kappa+1 \geq\left(\frac{4 C_{0}}{\mathfrak{\kappa}(T)}\right)^{2}$, this directly implies $(u, v) \in \mathcal{H}_{H F}^{s}(T)$.

\subsection{The full observability estimate}

Once the high-frequency observability estimate is proved, it remains to say something on the low-frequencies, i.e. remove the term $\mathscr{E}_{s-1 / 2}\left(V_{0}\right)$ in the right hand-side of (2.18) for general data (as opposed to the result in Cor. 2.6). This is based on [23]. We only use the case $s=1$ in (2.18) to which [23] is more adapted. As a corollary of Theorem 1.6 (i.e. [23], Thm. 1.1), we have the following intermediate estimates.

Corollary 2.8. Let $\omega_{0}$ be an open set of $M$ and fix $T_{0}>T_{U C}\left(\omega_{0}\right)$. Then, there exist $\kappa, \mu_{0}>0$ such that for any $s \in[0,1)$ there is $C>0$ such that for all $\left(v_{0}, v_{1}\right) \in \mathcal{H}^{1}(M)$ and associated solution $v \in \mathscr{C}^{0}\left(0, T ; H^{1}(M)\right)$ of (1.2), for any $T \geq T_{0}$ and $\mu \geq \mu_{0}^{1-s}$, we have

$$
\left\|\left(v_{0}, v_{1}\right)\right\|_{\mathcal{H}^{s}(M)} \leq C C_{s}(\mu)\|v\|_{L^{2}\left(0, T ; H^{1}\left(\omega_{0}\right)\right)}+\frac{C}{\mu}\left\|\left(v_{0}, v_{1}\right)\right\|_{\mathcal{H}^{1}(M)},
$$

with $C_{s}(\mu)=\mu^{\frac{s}{1-s}} \mathrm{e}^{\kappa \mu^{\frac{1}{1-s}}}$. In particular, for any $T \geq T_{0}$ and $\mu \geq \mu_{0}^{1 / 2}$, we have

$$
\left\|\left(v_{0}, v_{1}\right)\right\|_{\mathcal{H}^{1 / 2}(M)} \leq C \mu \mathrm{e}^{\kappa \mu^{2}}\|v\|_{L^{2}\left(0, T ; H^{1}\left(\omega_{0}\right)\right)}+\frac{C}{\mu}\left\|\left(v_{0}, v_{1}\right)\right\|_{\mathcal{H}^{1}(M)} .
$$

Proof. We denote by $V_{0}=\left(v_{0}, v_{1}\right)$ all along the proof. Using an interpolation estimate and Young inequality, with $\eta>0$, we obtain for $C>0$ (depending on $s$ )

$$
\left\|V_{0}\right\|_{\mathcal{H}^{s}(M)} \leq C\left\|V_{0}\right\|_{\mathcal{H}^{0}(M)}^{1-s}\left\|V_{0}\right\|_{\mathcal{H}^{1}(M)}^{s} \leq C(1-s) \eta^{-1 /(1-s)}\left\|V_{0}\right\|_{\mathcal{H}^{0}(M)}+C s \eta^{1 / s}\left\|V_{0}\right\|_{\mathcal{H}^{1}(M)} .
$$


Then using (1.16) for $T=T_{0}>T_{U C}\left(\omega_{0}\right)$ yields, for $\mu \geq \mu_{0}$,

$$
\left\|V_{0}\right\|_{\mathcal{H}^{s}(M)} \leq C \eta^{-1 /(1-s)}\left[C_{0}(\mu)\|v\|_{L^{2}\left(0, T_{0} ; H^{1}\left(\omega_{0}\right)\right)}+\frac{1}{\mu}\left\|V_{0}\right\|_{\mathcal{H}^{1}(M)}\right]+C \eta^{1 / s}\left\|V_{0}\right\|_{\mathcal{H}^{1}(M)}
$$

Now, we take $\eta$ such that $\eta^{\frac{1}{s(1-s)}}=\frac{1}{\mu}$, implying, for $\mu \geq \mu_{0}$,

$$
\left\|V_{0}\right\|_{\mathcal{H}^{s}(M)} \leq C \mu^{s} C_{0}(\mu)\|v\|_{L^{2}\left(0, T_{0} ; H^{1}\left(\omega_{0}\right)\right)}+\frac{C}{\mu^{1-s}}\left\|V_{0}\right\|_{\mathcal{H}^{1}(M)} .
$$

Finally, writing $\tilde{\mu}=\mu^{1-s}$, there is $C>0$ (depending on $s$ ) such that for any $\tilde{\mu} \geq \mu_{0}^{1-s}$, we have

$$
\left\|V_{0}\right\|_{\mathcal{H}^{s}(M)} \leq C \tilde{\mu}^{\frac{s}{1-s}} C_{0}\left(\tilde{\mu}^{\frac{1}{1-s}}\right)\|v\|_{L^{2}\left(0, T_{0} ; H^{1}\left(\omega_{0}\right)\right)}+\frac{C}{\tilde{\mu}}\left\|V_{0}\right\|_{\mathcal{H}^{1}(M)} .
$$

Using that $\|v\|_{L^{2}\left(0, T_{0} ; H^{1}\left(\omega_{0}\right)\right)} \leq\|v\|_{L^{2}\left(0, T ; H^{1}\left(\omega_{0}\right)\right)}$ for all $T \geq T_{0}$ concludes the proof of the corollary.

We can now conclude the proof of the main theorem in the model case of the Klein-Gordon equation by combining the high-frequency estimate (2.18) and the low-frequency estimate (2.23).

Proof of the observability Theorem 1.2. First, according to Lemma B.8 and the assumption $T_{U C}(\omega)<T_{G C C}(\omega)$, there is an open subset $\omega_{0}$ of $M$ such that

$$
\bar{\omega}_{0} \subset \omega, \text { and } T_{U C}\left(\omega_{0}\right)<T_{G C C}(\omega) .
$$

We now choose $T_{0}$, so that we have

$$
0<T_{U C}(\omega) \leq T_{U C}\left(\omega_{0}\right)<T_{0}<T_{G C C}(\omega)<T_{1}
$$

(note that the assumption $T_{U C}(\omega)<T_{G C C}(\omega)$ implies $T_{G C C}(\omega)>0$ and hence $\bar{\omega} \neq M$ and hence $T_{U C}\left(\omega_{0}\right) \geq$ $\left.T_{U C}(\omega)>0\right)$.

The high-frequency estimate (2.18) for $s=1$, yields the existence of $C_{0}>0$ such that for all $T \in\left[0, T_{1}\right]$, $V_{0}=\left(v_{0}, v_{1}\right)$, and associated solution $v \in \mathscr{C}^{0}\left(0, T ; H^{1}(M)\right)$ of $(1.2)$, we have

$$
\int_{0}^{T}\left\|b_{\omega} v(t)\right\|_{H^{1}(M)}^{2} \mathrm{~d} t \geq \mathfrak{K}(T) \mathscr{E}_{1}\left(V_{0}\right)-C_{0} \mathscr{E}_{1 / 2}\left(V_{0}\right) .
$$

The low-frequency estimate (2.23) (squared) gives the existence of $C, \kappa, \mu_{0}>0$, such that one has

$$
\mathscr{E}_{1 / 2}\left(V_{0}\right) \leq C \mu^{2} \mathrm{e}^{2 \kappa \mu^{2}} \int_{0}^{T}\|v\|_{H^{1}\left(\omega_{0}\right)}^{2} \mathrm{~d} t+\frac{C}{\mu^{2}} \mathscr{E}_{1}\left(V_{0}\right)
$$

for all $\mu \geq \mu_{0}^{\frac{1}{2}}$ and all $T \geq T_{0}$. Changing $\mu^{2}$ into $\mu$, these last two estimates yield, for any $\mu \geq \mu_{0}$ and $T \in\left[T_{0}, T_{1}\right]$ (the constant $C>0$ may change from line to line, but remains uniform with respect to the parameters $T$ and $\mu$ ),

$$
\int_{0}^{T}\left\|b_{\omega} v(t)\right\|_{H^{1}(M)}^{2} \mathrm{~d} t \geq \mathfrak{K}(T) \mathscr{E}_{1}\left(V_{0}\right)-C\left(\mathrm{e}^{\tilde{\kappa} \mu} \int_{0}^{T}\|v\|_{H^{1}\left(\omega_{0}\right)}^{2} \mathrm{~d} t+\frac{1}{\mu} \mathscr{E}_{1}\left(V_{0}\right)\right),
$$

that is

$$
\int_{0}^{T}\left\|b_{\omega} v(t)\right\|_{H^{1}(M)}^{2} \mathrm{~d} t+C \mathrm{e}^{\tilde{\kappa} \mu} \int_{0}^{T}\|v\|_{H^{1}\left(\omega_{0}\right)}^{2} \mathrm{~d} t \geq\left(\mathfrak{K}(T)-\frac{C}{\mu}\right) \mathscr{E}_{1}\left(V_{0}\right) .
$$


Assuming now that $T>T_{G C C}(\omega)$, we have $\mathfrak{K}(T)>0$, and may choose $\mu=\max \left\{\frac{2 C}{\mathfrak{K}(T)}, \mu_{0}\right\}$ to obtain, for some $\kappa^{*}>0, C>0$

$$
\int_{0}^{T}\left\|b_{\omega} v(t)\right\|_{H^{1}(M)}^{2} \mathrm{~d} t+C \mathrm{e}^{\kappa^{*} \max \left\{\mathfrak{K}(T)^{-1}, \mu_{0}\right\}} \int_{0}^{T}\|v\|_{H^{1}\left(\omega_{0}\right)}^{2} \mathrm{~d} t \geq \frac{\mathfrak{K}(T)}{2} \mathscr{E}_{1}\left(V_{0}\right)
$$

Note that until this point, we did not use the assumptions on the relative location of the sets $\omega_{0}$ and $\omega=\left\{b_{\omega} \neq 0\right\}$ (except that $\left.T_{U C}\left(\omega_{0}\right)<T_{G C C}(\omega)\right)$.

Finally, using that $\bar{\omega}_{0} \subset \omega=\left\{b_{\omega} \neq 0\right\}$, we have $\left|b_{\omega}\right| \geq c_{0}^{-1}>0$ on $\omega_{0}$ and $1=\frac{b_{\omega}}{b_{\omega}}$ on this set, so that

$$
\begin{aligned}
\|v\|_{H^{1}\left(\omega_{0}\right)}^{2} & =\int_{\omega_{0}}|\nabla v|^{2}+|v|^{2} \mathrm{~d} x=\int_{\omega_{0}}\left|\nabla\left(b_{\omega}^{-1} b_{\omega} v\right)\right|^{2}+\left|b_{\omega}^{-1} b_{\omega} v\right|^{2} \mathrm{~d} x \\
& \leq \int_{\omega_{0}}\left|\nabla b_{\omega}^{-1}\right|^{2}\left|b_{\omega} v\right|^{2}+c_{0}^{2} \int_{\omega_{0}}\left|\nabla\left(b_{\omega} v\right)\right|^{2}+\left|b_{\omega} v\right|^{2} \mathrm{~d} x \\
& \leq C \int_{\omega_{0}}\left|\nabla\left(b_{\omega} v\right)\right|^{2}+\left|b_{\omega} v\right|^{2} \mathrm{~d} x \leq C\left\|b_{\omega} v\right\|_{H^{1}(M)}^{2} .
\end{aligned}
$$

As a consequence, coming back to $(2.26)$, there is $C, \kappa^{\prime}>0$ such that for all $T \in\left(T_{G C C}(\omega), T_{1}\right]$, we have

$$
C \mathrm{e}^{\kappa^{\prime} \mathfrak{K}(T)^{-1}} \int_{0}^{T}\left\|b_{\omega} v(t)\right\|_{H^{1}(M)}^{2} \mathrm{~d} t \geq \mathscr{E}_{1}\left(V_{0}\right)
$$

which concludes the proof of Theorem 1.2.

\subsection{The high-frequency estimate in the general case}

We now consider the general case of the observability problem (1.2)-(1.10) (dual to the controllability problem (1.1)-(1.9)), and give a proof of Theorem 1.4. We only provide below the high-frequency part of the analysis. The analogue of Theorem 1.1 (the lower bound) directly follows (and does not require the analyticity of the coefficients). Concerning the analogue of Theorem 1.2 (the upper bound), its low-frequency part uses ([23], Thm. 6.1) (instead of Theorem 1.6 which only deals with $L^{*}=-\Delta$ ), which requires the coefficients to be analytic in time. The proof of the full observability estimate from the high-frequency one then follows Section 2.3, without any modification.

The main purpose of the following subsection is therefore the proof of Proposition 2.14 below, which is the generalization of Proposition 2.5. As in the Klein-Gordon case, the proof proceeds in several steps:

- Writing the equation as a $2 \times 2$ system.

- Using a trick due to Taylor to eliminate the anti-diagonal lower order terms, this is the object of Proposition 2.9.

- Applying an Egorov theorem to get a nice pseudodifferential representation. This is Proposition 2.12.

- Concluding by the Gårding inequality.

When performing the high-frequency analysis of this observation problem, it is convenient to recast it in a more general framework. More precisely, given a fixed time $T_{0}>0$, we shall study the HUM control operator for the problem

$$
\left\{\begin{array}{l}
\partial_{t}^{2} v-\Delta v+v+A_{0} D_{t} v+A_{1} v=0, \quad \text { on }\left[0, T_{0}\right] \times M \\
\left(v(0), \partial_{t} v(0)\right)=\left(v_{0}, v_{1}\right)
\end{array}\right.
$$

where $A_{0} \in \mathscr{C}^{\infty}\left(0, T_{0} ; \Psi_{\mathrm{phg}}^{0}(M)\right), D_{t}=\frac{\partial_{t}}{i}$ and $A_{1} \in \mathscr{C}^{\infty}\left(0, T_{0} ; \Psi_{\mathrm{phg}}^{1}(M)\right)$ have symbols

$$
a_{0}=\sigma_{0}\left(A_{0}\right) \in \mathscr{C}^{\infty}\left(0, T_{0} ; S_{\mathrm{phg}}^{0}(M)\right), \quad a_{1}=\sigma_{1}\left(A_{1}\right) \in \mathscr{C}^{\infty}\left(0, T_{0} ; S_{\mathrm{phg}}^{1}(M)\right) .
$$


The main additional difficulty with respect to the model case of Section 2.2 is that we do not have the simple representation formula (2.7) for the solution.

The equation (1.2)-(1.10) under interest is a particular case of (2.27) with

$$
\begin{array}{r}
A_{0}=-i \overline{b_{0}}, \quad \text { with } a_{0}(t, x, \xi)=-i \overline{b_{0}}(t, x), \\
A_{1}=-\left\langle d \cdot \overline{b_{1}}\right\rangle_{x}+\left(\bar{c}-\partial_{t} \overline{b_{0}}-\operatorname{div}\left(\overline{b_{1}}\right)\right), \quad \text { with } a_{1}(t, x, \xi)=-i\left\langle\xi, \overline{b_{1}}(t, x)\right\rangle_{x},
\end{array}
$$

and all (high-frequency) results proved for (2.27) yield a counterpart for (1.2)-(1.10).

We now focus on equation $(2.27)$. For $\left(v_{0}, v_{1}\right) \in H^{s} \times H^{s-1}$, we recall that there exists a unique solution $v \in \mathscr{C}^{0}\left(0, T_{0} ; H^{s}(M)\right) \cap \mathscr{C}^{1}\left(0, T_{0} ; H^{s-1}(M)\right)$ to $(2.27)$. We set

$$
v^{+}(t)=\left(D_{t}+\Lambda\right) v(t), \quad v^{-}(t)=\left(D_{t}-\Lambda\right) v(t)
$$

so that $v^{ \pm} \in \mathscr{C}^{0}\left(0, T_{0} ; H^{s-1}(M)\right)$ for $\left(v_{0}, v_{1}\right) \in H^{s} \times H^{s-1}$. We have

$$
v(t)=\frac{1}{2} \Lambda^{-1}\left(v^{+}(t)-v^{-}(t)\right), \quad D_{t} v(t)=\frac{1}{2}\left(v^{+}(t)+v^{-}(t)\right) .
$$

This corresponds to the splitting $\left(v^{+}, v^{-}\right)=\widetilde{\Sigma}\left(v, \partial_{t} v\right)$ with

$$
\widetilde{\Sigma}=\left(\begin{array}{cc}
\Lambda & 1 / i \\
-\Lambda & 1 / i
\end{array}\right), \quad \widetilde{\Sigma}^{-1}=\frac{1}{2}\left(\begin{array}{cc}
\Lambda^{-1} & -\Lambda^{-1} \\
i & i
\end{array}\right) .
$$

Note that this is not exactly the splitting $\Sigma$ introduced in (2.4) but we have

$$
\Sigma=\frac{1}{2} \Lambda^{-1}\left(\begin{array}{cc}
1 & 0 \\
0 & -1
\end{array}\right) \widetilde{\Sigma}
$$

We could also have performed the analysis in Section 2.2 with $\widetilde{\Sigma}$, but in the case of the Klein-Gordon equation, $\Sigma$ was more convenient to work with in $H^{s} \times H^{s}$.

Then, writing $\partial_{t}^{2}-\Delta+1=-\left(D_{t}+\Lambda\right)\left(D_{t}-\Lambda\right)$, equation (2.27) can be recast as a system of two first order hyperbolic equation in terms of $v^{ \pm}$, namely

$$
\left\{\begin{array}{l}
-\left(D_{t}-\Lambda\right) v^{+}+\frac{A_{0}}{2}\left(v^{+}+v^{-}\right)+\frac{A_{1} \Lambda^{-1}}{2}\left(v^{+}-v^{-}\right)=0 \\
-\left(D_{t}+\Lambda\right) v^{-}+\frac{A_{0}}{2}\left(v^{+}+v^{-}\right)+\frac{A_{1} \Lambda^{-1}}{2}\left(v^{+}-v^{-}\right)=0 .
\end{array}\right.
$$

This is a striclty hyperbolic Cauchy problem ([52], Chap. 7.7) with solution operator $\mathscr{S}(t, s)$. As in the scalar case (see Cor. A.2), it enjoys the regularity

$$
\begin{array}{r}
\mathscr{S}(t, s) \in \mathcal{B}\left(\left(0, T_{0}\right)^{2} ; \mathcal{L}\left(H^{\sigma}\left(M ; \mathbb{C}^{2}\right)\right)\right), \\
\partial_{t} \mathscr{S}(t, s), \partial_{s} \mathscr{S}(t, s) \in \mathcal{B}\left(\left(0, T_{0}\right)^{2} ; \mathcal{L}\left(H^{\sigma}\left(M ; \mathbb{C}^{2}\right) ; H^{\sigma-1}\left(M ; \mathbb{C}^{2}\right)\right)\right),
\end{array}
$$

for all $\sigma \in \mathbb{R}$. The definition of the operators $\partial_{t} \mathscr{S}(t, s), \partial_{s} \mathscr{S}(t, s)$ is given in Corollary A.2 (in the scalar case). It can be rewritten as

$$
\left\{\begin{array}{l}
\left(D_{t}-\Lambda\right) v^{+}-A_{+} v^{+}-A_{-} v^{-}=0, \\
\left(D_{t}+\Lambda\right) v^{-}-A_{+} v^{+}-A_{-} v^{-}=0,
\end{array}\right.
$$

with

$$
A_{+}=\frac{1}{2}\left(A_{0}+A_{1} \Lambda^{-1}\right), \quad A_{-}=\frac{1}{2}\left(A_{0}-A_{1} \Lambda^{-1}\right),
$$


both belonging to $\mathscr{C}^{\infty}\left(0, T_{0} ; \Psi_{\mathrm{phg}}^{0}(M)\right)$. Note that the equations are only coupled by zero order terms. Again, this is $P V=0$ with $V={ }^{t}\left(v^{+}, v^{-}\right)$and

$$
P=D_{t}+M-A, \quad M=\left(\begin{array}{cc}
-\Lambda & 0 \\
0 & \Lambda
\end{array}\right), \quad A=\left(\begin{array}{c}
A_{+} A_{-} \\
A_{+} A_{-}
\end{array}\right) .
$$

With this splitting in hand, we first have the following high-frequency representation formula for solutions of $(2.27)$ or $(2.34)$.

Proposition 2.9. We denote by $S_{ \pm}(t, s)$ the solution operator associated to $\left(\partial_{t} \pm i \Lambda-i A_{ \pm}\right)$, that is $y\left(s^{\prime}\right)=$ $S_{ \pm}\left(s^{\prime}, s\right) y(s)$ if and only if

$$
\left(\partial_{t} \pm i \Lambda-i A_{ \pm}(t)\right) y(t)=0, \quad \text { for all } t \in\left[s, s^{\prime}\right] .
$$

We also define

$$
\mathcal{S}(t, s)=\left(\begin{array}{cc}
S_{+}(t, s) & 0 \\
0 & S_{-}(t, s)
\end{array}\right) .
$$

Then the solution operator $\mathscr{S}(t, s)$ of (2.34) satisfies

$$
\mathscr{S}(t, s)=\mathcal{S}(t, s)+\mathcal{R}(t, s), \quad(t, s) \in\left[0, T_{0}\right]^{2},
$$

where, for all $\sigma \in \mathbb{R}$,

$$
\begin{array}{r}
\mathcal{R}(t, s) \in \mathcal{B}\left(\left(0, T_{0}\right)^{2} ; \mathcal{L}\left(H^{\sigma}\left(M ; \mathbb{C}^{2}\right) ; H^{\sigma+1}\left(M ; \mathbb{C}^{2}\right)\right)\right) \\
\partial_{t} \mathcal{R}(t, s), \partial_{s} \mathcal{R}(t, s) \in \mathcal{B}\left(\left(0, T_{0}\right)^{2} ; \mathcal{L}\left(H^{\sigma}\left(M ; \mathbb{C}^{2}\right)\right)\right)
\end{array}
$$

Proof of Proposition 2.9. We use a trick (due to Taylor [51], Sect. 2) to decouple the equations. More precisely, we look for $K \in \mathscr{C}^{\infty}\left(0, T_{0} ; \Psi_{\text {phg }}^{-1}\left(M ; \mathbb{C}^{2}\right)\right)$ so that the function $W=(\operatorname{Id}-K) V$ solves a diagonal system, up to appropriate remainders (on the variable $V$ ). We have on the one hand

$$
(\mathrm{Id}+K) W=V-K^{2} V,
$$

and hence

$$
(\mathrm{Id}-K) P(\operatorname{Id}+K) W=(\mathrm{Id}-K) P\left(V-K^{2} V\right)=-(\mathrm{Id}-K) P K^{2} V=R V,
$$

since $P V=0$. Moreover, the remainder satisfies $R \in \mathscr{R}^{-1}$, where

$$
\mathscr{R}^{-1}=\mathscr{C}^{\infty}\left(0, T_{0} ; \Psi_{\mathrm{phg}}^{-1}\left(M ; \mathbb{C}^{2}\right)\right)+\mathscr{C}^{\infty}\left(0, T_{0} ; \Psi_{\mathrm{phg}}^{-2}\left(M ; \mathbb{C}^{2}\right)\right) D_{t}
$$

is the admissible class of remainders in the present context. On the other hand, we have

$$
(\mathrm{Id}-K) P(\operatorname{Id}+K) W=P W+[P, K] W-K P K W,
$$

with $K P K \in \mathscr{R}^{-1}$. We then remark that $\left[D_{t}, K\right] W=\left(D_{t} K\right) W$ so that $\left[D_{t}, K\right] \in \mathscr{C}^{\infty}\left(0, T_{0} ; \Psi_{\mathrm{phg}}^{-1}\left(M ; \mathbb{C}^{2}\right)\right) \subset$ $\mathscr{R}^{-1}$, and as well $[A, K] \in \mathscr{R}^{-1}$. Hence, if we can find $K$ such that

$$
-\left(\begin{array}{cc}
0 & A_{-} \\
A_{+} & 0
\end{array}\right)+[M, K] \in \mathscr{R}^{-1}
$$


we will then obtain from (2.40) and (2.41) that $W$ solves

$$
P_{d} W=R_{1} W+R_{2} V=R V,
$$

with $R_{1}, R_{2}, R \in \mathscr{R}^{-1}$ and, with $M$ defined in (2.35),

$$
P_{d}=D_{t}+M-A_{d}, \quad A_{d}=\left(\begin{array}{cc}
A_{+} & 0 \\
0 & A_{-}
\end{array}\right) .
$$

Now taking (for instance)

$$
K:=\frac{1}{2}\left(\begin{array}{cc}
0 & -\Lambda^{-1} A_{+} \\
A_{-} \Lambda^{-1} & 0
\end{array}\right) \in \mathscr{C}^{\infty}\left(0, T_{0} ; \Psi_{\text {phg }}^{-1}\left(M ; \mathbb{C}^{2}\right)\right)
$$

realizes (2.42), and we are left to study $P_{d} W=R V, R \in \mathscr{R}^{-1}$, with $W=(\operatorname{Id}-K) V$.

With $\mathcal{S}(t, s)$ defined in (2.37), equation (2.43) is now solved by

$$
W(t)=\mathcal{S}(t, s) W(s)+\int_{s}^{t} \mathcal{S}\left(t, t^{\prime}\right) R\left(t^{\prime}\right) V\left(t^{\prime}\right) \mathrm{d} t^{\prime}, \quad R \in \mathscr{R}^{-1} .
$$

Recalling that $W=(\operatorname{Id}-K) V$ and that $V(t)=\mathscr{S}(t, s) V(s)$, this yields

$$
V(t)=\mathcal{S}(t, s) V(s)+K(t) \mathscr{S}(t, s) V(s)-\mathcal{S}(t, s) K(s) V(s)+\left(\int_{s}^{t} \mathcal{S}\left(t, t^{\prime}\right) R\left(t^{\prime}\right) \mathscr{S}\left(t^{\prime}, s\right) \mathrm{d} t^{\prime}\right) V(s) .
$$

This can be rewritten as

$$
V(t)=\mathcal{S}(t, s) V(s)+\mathcal{R}(t, s) V(s)
$$

with

$$
\mathcal{R}(t, s)=K(t) \mathscr{S}(t, s)-\mathcal{S}(t, s) K(s)+\left(\int_{s}^{t} \mathcal{S}\left(t, t^{\prime}\right) R\left(t^{\prime}\right) \mathscr{S}\left(t^{\prime}, s\right) \mathrm{d} t^{\prime}\right)
$$

satisfying

$$
\begin{array}{r}
\mathcal{R}(t, s) \in \mathcal{B}\left(\left(0, T_{0}\right)^{2} ; \mathcal{L}\left(H^{\sigma}\left(M ; \mathbb{C}^{2}\right) ; H^{\sigma+1}\left(M ; \mathbb{C}^{2}\right)\right)\right), \\
\partial_{t} \mathcal{R}(t, s), \partial_{s} \mathcal{R}(t, s) \in \mathcal{B}\left(\left(0, T_{0}\right)^{2} ; \mathcal{L}\left(H^{\sigma}\left(M ; \mathbb{C}^{2}\right)\right)\right),
\end{array}
$$

for all $\sigma \in \mathbb{R}$, according to the respective regularity properties of $\mathscr{S}(t, s), \mathcal{S}(t, s)$ and $K(s)$ (see Appendix A.1 for the regularity properties of $\mathcal{S}(t, s), \mathscr{S}(t, s))$.

Remark 2.10. Note that the decoupling of the two equations is permitted since the difference of the two eigenvalues of the principal part of the system, namely $\pm \lambda$, is elliptic. Moreover, we do no have the choice of the principal symbol of $K$ in this procedure. Also, we could choose $K$ by a classical iterative procedure so that all remainders are infinitely smoothing, which is not needed here.

Remark 2.11. Note here that we do not need to use that $\Lambda$ (the square root of the Laplace operator defined via spectral theory) is a pseudodifferential operator. Indeed, we could in place of $\Lambda$ use any operator $E$ such that

- $E \in \Psi_{\text {phg }}^{1}(M)$ with $\sigma_{1}(E)(x, \xi)=\lambda(x, \xi)=|\xi|_{x}$

- $E$ is selfadjoint on $L^{2}(M)$,

- $E$ is positive, in the sense that $(E u, u)_{L^{2}(M)} \geq C\|u\|_{L^{2}(M)}^{2}$, 
Then notice that we have $-\Delta-E^{2} \in \Psi_{\mathrm{phg}}^{1}(M)$, with principal symbol $\sigma_{1}\left(-\Delta-E^{2}\right)$ real since $-\Delta-E^{2}$ is selfadjoint on $L^{2}(M)$. As a consequence writing equation (2.27) with $E^{2}$ instead of $\Lambda^{2}=-\Delta+1$ only amounts to add to $A_{1}$ a term with real principal symbol. Then, we conclude by remarking that the result of Proposition 2.12 only depends on $\operatorname{Im}\left(a_{1}\right)$.

Such an operator $E$ is easy to construct using only basic pseudodifferential calculus on $M$ : Start with some $A \in$ $\Psi_{\mathrm{phg}}^{1}(M)$ with $\sigma_{1}(A)(x, \xi)=\lambda(x, \xi)$ (given by any quantification of the symbol $\lambda$ ), and set $E:=\frac{1}{2}\left(A+A^{*}\right)+C_{0}$ with $C_{0}$ large enough so that $E$ is positive (use for that the Gårding inequality). Then it is clear that $E$ fulfills all above conditions.

In Section 2.2, it was convenient to take an exact square root $\Lambda$, so that to have the nice exact formula $(2.7)$. The analysis of the present section shows this is not needed.

The representation formula of Proposition 2.9 together with an appropriate Egorov theorem (Thm. A.3) allows to express the Gramian control operator as follows.

Proposition 2.12. Denoting by $V_{0}=\left(v_{0}, v_{1}\right) \in H^{s}(M) \times H^{s-1}(M)$ the initial data for System (2.27), and $\widetilde{\Sigma} V_{0}={ }^{t}\left(\frac{v_{1}}{i}+\Lambda v_{0}, \frac{v_{1}}{i}-\Lambda v_{0}\right)$, we have

$$
\int_{0}^{T}\left\|b_{\omega} v(t)\right\|_{H^{s}(M)}^{2} \mathrm{~d} t=\left(\mathcal{G}_{T} \widetilde{\Sigma} V_{0}, \widetilde{\Sigma} V_{0}\right)_{H^{s-1}(M) \times H^{s-1}(M)},
$$

where $\mathcal{G}_{T}=G_{T}+R_{T}$ with $R_{T} \in \mathcal{B}\left(0, T_{0} ; \mathcal{L}\left(H^{\sigma}(M), H^{\sigma+1}\left(M ; \mathbb{C}^{2}\right)\right)\right)$ for all $\sigma \in \mathbb{R}$, and $G_{T} \in$ $\mathscr{C}^{\infty}\left(0, T_{0} ; \Psi_{\mathrm{phg}}^{0}\left(M ; \mathbb{C}^{2 \times 2}\right)\right)$ has principal symbol

$$
\sigma_{0}\left(G_{T}\right):=\frac{1}{4}\left(\begin{array}{cc}
g_{T}^{+} & 0 \\
0 & g_{T}^{-}
\end{array}\right) \in S_{\mathrm{phg}}^{0}\left(T^{*} M, \mathbb{C}^{2 \times 2}\right),
$$

with

$$
g_{T}^{ \pm}(\rho)=\int_{0}^{T} b_{\omega}^{2} \circ \pi \circ \varphi_{t}^{ \pm}(\rho) \mathrm{e}^{-\int_{0}^{t} \operatorname{Im}\left(a_{0} \pm a_{1} \lambda^{-1}\right)\left(\tau, \varphi_{\tau}^{ \pm}(\rho)\right) d \tau} \mathrm{d} t .
$$

Remark 2.13. Note that the proof of Proposition 2.12 also allows to recover an analogue of ([25], Lem. 3.1) which is the crucial step towards the estimate of the optimal exponential decay rate for the damped wave equation. Namely, for all $T>0$, there is a constant $C>0$ such that we have, for all solutions of $\partial_{t}^{2} v-\Delta v+b_{0} \partial_{t} v=$ 0 with real valued $b_{0}$, the following estimates:

$$
\begin{aligned}
& \mathscr{E}_{1}\left(v, \partial_{t} v\right)(T) \leq \exp \left(-2 \inf _{(x, \xi) \in S^{*} M} \int_{0}^{T} b_{0}(s, x(s)) \mathrm{d} s\right) \mathscr{E}_{1}\left(v, \partial_{t} v\right)(0)+C \mathscr{E}_{0}\left(v, \partial_{t} v\right)(0), \\
& \mathscr{E}_{1}\left(v, \partial_{t} v\right)(T) \geq \exp \left(-2 \sup _{(x, \xi) \in S^{*} M} \int_{0}^{T} b_{0}(s, x(s)) \mathrm{d} s\right) \mathscr{E}_{1}\left(v, \partial_{t} v\right)(0)-C \mathscr{E}_{0}\left(v, \partial_{t} v\right)(0),
\end{aligned}
$$

where $x(s)=\pi \circ \varphi_{s}(x, \xi)$. The proof is very close to that of Proposition 2.12: it follows from the representation formula of Proposition 2.9, the Egorov Theorem A.3, and the sharp Gårding estimate.

Proof of Proposition 2.12. According to (2.31), the unique solution to (2.27) is given by

$$
v(t)=\frac{1}{2} \Lambda^{-1}\left(v^{+}(t)-v^{-}(t)\right)=L V(t)
$$

where

$$
V(t)={ }^{t}\left(v^{+}(t), v^{-}(t)\right), \quad \text { and } \quad L:=\frac{1}{2} \Lambda^{-1}(1,-1)
$$


According to Proposition 2.9, $V(t)={ }^{t}\left(v^{+}(t), v^{-}(t)\right)$ satisfies

$$
V(t)=\mathscr{S}(t, 0) V^{0}, \quad \mathscr{S}(t, 0)=\mathcal{S}(t, 0)+\mathcal{R}(t, 0), \quad t \in\left[0, T_{0}\right],
$$

with

$$
V^{0}=\left(v_{0}^{+}, v_{0}^{-}\right)=\widetilde{\Sigma}\left(v_{0}, v_{1}\right)=\left(\frac{v_{1}}{i}+\Lambda v_{0}, \frac{v_{1}}{i}-\Lambda v_{0}\right) \in H^{s-1}\left(M ; \mathbb{C}^{2}\right),
$$

for $\left(v_{0}, v_{1}\right) \in H^{s}(M) \times H^{s-1}(M)$. Now, we compute

$$
\begin{aligned}
\int_{0}^{T}\left\|b_{\omega} v(t)\right\|_{H^{s}(M)}^{2} \mathrm{~d} t & =\int_{0}^{T}\left\|b_{\omega} L \mathscr{S}(t, 0) V^{0}\right\|_{H^{s}(M)}^{2} \mathrm{~d} t \\
& =\int_{0}^{T}\left(\mathscr{S}(t, 0)^{* t} L b_{\omega} \Lambda^{2 s} b_{\omega} L \mathscr{S}(t, 0) V^{0}, V^{0}\right)_{L^{2}\left(M ; \mathbb{C}^{2}\right)} \mathrm{d} t
\end{aligned}
$$

where all adjoints are taken in $L^{2}$. This implies

$$
\int_{0}^{T}\left\|b_{\omega} v(t)\right\|_{H^{s}(M)}^{2} \mathrm{~d} t=\left(\mathcal{G}_{T} V^{0}, V^{0}\right)_{H^{s-1}\left(M ; \mathbb{C}^{2}\right)}, \quad \mathcal{G}_{T}=\int_{0}^{T} \Lambda^{2(1-s)} \mathscr{S}(t, 0)^{* t} L b_{\omega} \Lambda^{2 s} b_{\omega} L \mathscr{S}(t, 0) \mathrm{d} t .
$$

Recalling now the form of $\mathscr{S}(t, 0)=\mathcal{S}(t, 0)+\mathcal{R}(t, 0)$ given by Proposition 2.9, we set

$$
\tilde{\mathcal{G}}_{T}:=\int_{0}^{T} \Lambda^{2(1-s)} \mathcal{S}(t, 0)^{* t} L b_{\omega} \Lambda^{2 s} b_{\omega} L \mathcal{S}(t, 0) \mathrm{d} t, \quad \text { and } \quad \tilde{R}_{T}=\mathcal{G}_{T}-\tilde{\mathcal{G}}_{T}
$$

The regularity properties of $\Lambda^{2(1-s)}, \mathscr{S}(t, 0), L$, and that of $\mathcal{R}(t, 0)$ given in (2.38)-(2.39) yield that

$$
\tilde{R}_{T} \in \mathcal{B}\left(\left(0, T_{0}\right) ; \mathcal{L}\left(H^{\sigma}\left(M ; \mathbb{C}^{2}\right) ; H^{\sigma+1}\left(M ; \mathbb{C}^{2}\right)\right)\right) .
$$

Next, recalling the definition of $\mathcal{S}(t, 0)$ in $(2.37)$, we can compute

$$
\mathcal{S}(t, 0)^{* t} L b_{\omega} \Lambda^{2 s} b_{\omega} L \mathcal{S}(t, 0)=\left(\begin{array}{cc}
S_{+}(t, 0)^{*} B S_{+}(t, 0) & -S_{+}(t, 0)^{*} B S_{-}(t, 0) \\
-S_{-}(t, 0)^{*} B S_{+}(t, 0) & S_{-}(t, 0)^{*} B S_{-}(t, 0)
\end{array}\right),
$$

with

$$
B=\frac{1}{4} \Lambda^{-1} b_{\omega} \Lambda^{2 s} b_{\omega} \Lambda^{-1} \in \Psi_{\mathrm{phg}}^{2 s-2}(M) .
$$

Let us first study the diagonal terms in (2.46). With $S_{ \pm}(t, 0)$ defined in (2.36), the Egorov Theorem A.3 yields the existence of $Q_{ \pm}(t) \in \mathscr{C}^{\infty}\left(\left(0, T_{0}\right), \Psi_{\mathrm{phg}}^{2 s-2}(M)\right)$ and

$$
\begin{gathered}
R_{ \pm}(t) \in \mathcal{B}\left(\left(0, T_{0}\right), \mathcal{L}\left(H^{\sigma}(M), H^{\sigma+1-2(s-1)}(M)\right)\right), \\
\partial_{t} R_{ \pm}(t) \in \mathcal{B}\left(\left(0, T_{0}\right), \mathcal{L}\left(H^{\sigma}(M), H^{\sigma-2(s-1)}(M)\right)\right),
\end{gathered}
$$

for all $\sigma \in \mathbb{R}$, such that we have

$$
S_{ \pm}(t, 0)^{*} B S_{ \pm}(t, 0)-Q_{ \pm}(t)=R_{ \pm}(t), \quad t \in\left(0, T_{0}\right) .
$$

and the principal symbol of $Q_{ \pm}(t)$ is given by

$$
q_{ \pm}(t, \rho)=\frac{1}{4} \lambda^{2 s-2} b_{\omega}^{2} \circ \pi \circ \varphi_{t}^{ \pm}(\rho) \mathrm{e}^{2 \int_{t}^{0} \operatorname{Im}\left(a_{ \pm}\right)\left(\tau, \varphi_{\tau}^{ \pm}(\rho)\right) d \tau} \in \mathscr{C}^{\infty}\left(\left(0, T_{0}\right), S_{\mathrm{phg}}^{2 s-2}\left(T^{*} M\right)\right),
$$

where $a_{ \pm}=\sigma_{0}\left(A_{ \pm}\right)$. 
Concerning the anti-diagonal terms in (2.46) when integrated on $(0, T)$, Lemma A.7 yields

$$
\int_{0}^{T} S_{ \pm}(t, 0)^{*} B S_{\mp}(t, 0) \mathrm{d} t \in \mathcal{B}\left(\left(0, T_{0}\right), \mathcal{L}\left(H^{\sigma}(M), H^{\sigma+1-2(s-1)}(M)\right)\right) .
$$

With all these properties in hand, when coming back to $(2.45)$, we may now write $\tilde{\mathcal{G}}_{T}:=G_{T}+R_{T}^{0}$ where $R_{T}^{0} \in \mathcal{B}\left(\left(0, T_{0}\right), \mathcal{L}\left(H^{\sigma}\left(M ; \mathbb{C}^{2}\right), H^{\sigma+1}\left(M ; \mathbb{C}^{2}\right)\right)\right)$ for all $\sigma \in \mathbb{R}$, and $G_{T}$ is given by

$$
G_{T}:=\left(\begin{array}{cc}
\Lambda^{2(1-s)} \int_{0}^{T} Q_{+}(t) \mathrm{d} t & 0 \\
0 & \Lambda^{2(1-s)} \int_{0}^{T} Q_{-}(t) \mathrm{d} t
\end{array}\right) \quad \in \mathscr{C}^{\infty}\left(0, T_{0} ; \Psi_{\mathrm{phg}}^{0}(M)\right),
$$

and has principal symbol

$$
\sigma_{0}\left(G_{T}\right):=\frac{1}{4}\left(\begin{array}{cc}
\int_{0}^{T} b_{\omega}^{2} \circ \pi \circ \varphi_{t}^{+}(\rho) \mathrm{e}^{-2 \int_{0}^{t} \operatorname{Im}\left(a_{+}\right)\left(\tau, \varphi_{\tau}^{+}(\rho)\right) d \tau} \mathrm{d} t & 0 \\
0 & \int_{0}^{T} b_{\omega}^{2} \circ \pi \circ \varphi_{t}^{-}(\rho) \mathrm{e}^{-2 \int_{0}^{t} \operatorname{Im}\left(a_{-}\right)\left(\tau, \varphi_{\tau}^{-}(\rho)\right) d \tau} \mathrm{d} t
\end{array}\right) .
$$

This, together with (2.45) concludes the proof of the proposition.

As a consequence of Proposition 2.12, we obtain the following high-frequency observability estimate. We use for this the definition of the constant $\mathfrak{K}(T)$ associated to (2.27):

$$
\mathfrak{K}(T):=\min \left\{\min _{\rho \in S^{*} M} g_{T}^{+}(\rho), \min _{\rho \in S^{*} M} g_{T}^{-}(\rho)\right\} .
$$

Proposition 2.14. For any $T_{0}>0$, there exists a constant $C_{0}>0$ such that for all $T \in\left[0, T_{0}\right]$, for all $V_{0}=\left(v_{0}, v_{1}\right) \in H^{s}(M) \times H^{s-1}(M)$ and associated solution $v$ of $(2.27)$, we have

$$
\int_{0}^{T}\left\|b_{\omega} v(t)\right\|_{H^{s}(M)}^{2} \mathrm{~d} t \geq \mathfrak{K}(T) \mathscr{E}_{s}\left(V_{0}\right)-C_{0} \mathscr{E}_{s-1 / 2}\left(V_{0}\right),
$$

where $\mathfrak{K}(T)$ is defined by $(2.47)$.

Note that in the case of equation (1.2)-(1.10) above, the symbols $a_{0}, a_{1}$ are given by (2.28) and (2.29), so that in this case, denoting by $\left(x^{ \pm}(s), \xi^{ \pm}(s)\right)=\varphi_{s}^{ \pm}\left(x_{0}, \xi_{0}\right)$, we have

$$
g_{T}^{ \pm}\left(x_{0}, \xi_{0}\right)=\int_{0}^{T} b_{\omega}^{2}\left(x^{ \pm}(t)\right) \exp \left(\int_{0}^{t} \operatorname{Re}\left(b_{0}\right)\left(\tau, x^{ \pm}(\tau)\right) \pm\left\langle\frac{\xi^{ \pm}(\tau)}{\left|\xi^{ \pm}(\tau)\right|_{x^{ \pm}(\tau)}}, \operatorname{Re}\left(b_{1}\right)\left(\tau, x^{ \pm}(\tau)\right)\right\rangle_{x^{ \pm}(\tau)} \mathrm{d} \tau\right) \mathrm{d} t .
$$

The two functions $g_{T}^{-}$and $g_{T}^{+}$in (2.49) are linked by the following lemma, proved in Appendix B.

Lemma 2.15. With $g_{T}^{-}$and $g_{T}^{+}$given by (2.49) we have $g_{T}^{-} \circ \sigma=g_{T}^{+}$, where $\sigma(x, \xi)=(x,-\xi)$.

According to Lemma 2.15 (together with the fact that $\sigma$ is an involution), we have in this situation $\min _{\rho \in S^{*} M} g_{T}^{+}(\rho)=\min _{\rho \in S^{*} M} g_{T}^{-}(\rho)$. This justifies the definition (1.11) in Theorem 1.4.

Proof of Proposition 2.14. We follow the proof of Proposition 2.5. From Proposition 2.12 and the use of the uniform Gårding estimate of Theorem A.9 (or its corollary), we obtain, uniformly for $T \in\left[0, T_{0}\right]$,

$$
\begin{aligned}
\int_{0}^{T}\left\|b_{\omega} v(t)\right\|_{H^{s}(M)}^{2} \mathrm{~d} t & =\left(\mathcal{G}_{T} \widetilde{\Sigma} V_{0}, \widetilde{\Sigma} V_{0}\right)_{H^{s-1}(M) \times H^{s-1}(M)} \\
& \geq \frac{1}{4} \mathfrak{K}(T)\left\|\widetilde{\Sigma} V_{0}\right\|_{H^{s-1}\left(M ; \mathbb{C}^{2}\right)}^{2}-C_{0}\left\|\widetilde{\Sigma} V_{0}\right\|_{H^{s-3 / 2}\left(M ; \mathbb{C}^{2}\right)}^{2} .
\end{aligned}
$$

To conclude, we just notice that

$$
\begin{aligned}
\left\|\widetilde{\Sigma} V_{0}\right\|_{H^{s-1}\left(M ; \mathbb{C}^{2}\right)}^{2} & =\left\|\frac{v_{1}}{i}+\Lambda v_{0}\right\|_{H^{s-1}(M)}^{2}+\left\|\frac{v_{1}}{i}-\Lambda v_{0}\right\|_{H^{s-1}(M)}^{2} \\
& =2\left\|v_{1}\right\|_{H^{s-1}(M)}^{2}+2\left\|v_{0}\right\|_{H^{s}(M)}^{2}=4 \mathscr{E}_{s}\left(V_{0}\right) .
\end{aligned}
$$




\section{UNIFORM DEPENDENCE WITH RESPECT TO POTENTIALS}

In this section, we allow $M$ to have a nonempty boundary $\partial M$. In fact, we do not perform a high-frequency analysis but rather use as a black box a known result, for which we refer, e.g., to [2] or [25]. We hence now use the notation: $\mathcal{H}^{1}=H_{0}^{1}(M) \times L^{2}(M), \mathcal{H}^{1}=L^{2}(M) \times H^{-1}(M)\left(H^{-1}\right.$ being the usual dual space of $\left.H_{0}^{1}\right)$, and

$$
\mathscr{E}_{1}\left(u, \partial_{t} u\right)=\frac{1}{2}\left(\left\|\partial_{t} u\right\|_{L^{2}(M)}^{2}+\|\nabla u\|_{L^{2}(M)}^{2}+\|u\|_{L^{2}(M)}^{2}\right) .
$$

In Section 3.1, we first focus on obaining (from [23]) an explicit dependence of the low frequency estimates with respect to potentials. We then conclude the proof in Section 3.2.

\subsection{The low-frequency estimate}

Our starting point is the following result, which is a particular case of ([23], Thm. 6.3) (in which the operator considered may also contain first order terms).

Theorem 3.1. For any nonempty open subset $\omega$ of $M$ and any $T>\mathcal{L}(M, \omega)$, there exist $\varepsilon, C, \kappa, \mu_{0}>0$ such that for any $c \in L^{\infty}(M)$, any $u \in H^{1}((-T, T) \times M)$ solving (1.13), we have, for any $\mu \geq \mu_{0} \max \left\{1,\|c\|_{L^{\infty}}^{\frac{2}{3}}\right\}$,

$$
\|u\|_{L^{2}((-\varepsilon, \varepsilon) \times M)} \leq C \mathrm{e}^{\kappa \mu}\|u\|_{L^{2}\left((-T, T) ; H^{1}(\omega)\right)}+\frac{C}{\mu}\|u\|_{H^{1}((-T, T) \times M)} .
$$

If $\partial M \neq \emptyset$ and $\Gamma$ is a non empty open subset of $\partial M$, for any $T>\mathcal{L}(M, \Gamma)$, there exist $\varepsilon, C, \kappa, \mu_{0}>0$ such that for any $u \in H^{1}((-T, T) \times M)$ solving (1.13), we have

$$
\|u\|_{L^{2}((-\varepsilon, \varepsilon) \times M)} \leq C \mathrm{e}^{\kappa \mu}\left\|\partial_{\nu} u\right\|_{L^{2}((-T, T) \times \Gamma)}+\frac{C}{\mu}\|u\|_{H^{1}((-T, T) \times M)} .
$$

From this result, we may deduce, in case there is no first order terms, the following corollary which is a refined version of ([23], Thm. 6.1) (in which we replace $C=C_{0} \mathrm{e}^{C_{0}\|c\|_{L} \infty}$ by $C=C_{0} \mathrm{e}^{C_{0} \sqrt{\|c\|_{L} \infty}}$ )

Corollary 3.2. Under the same assumptions as Theorem 3.1, there exist $C_{0}, \kappa, \mu_{0}>0$ such that for any $c \in L^{\infty}(M)$, any $u \in H^{1}((-T, T) \times M)$ solving (1.13), we have, for any $\mu \geq \mu_{0} \max \left\{1,\|c\|_{L^{\infty}}^{\frac{2}{3}}\right\}$,

$$
\left\|\left(u_{0}, u_{1}\right)\right\|_{\mathcal{H}^{0}} \leq C \mathrm{e}^{\kappa \mu}\|u\|_{L^{2}\left((-T, T) ; H^{1}(\omega)\right)}+\frac{C}{\mu}\left\|\left(u_{0}, u_{1}\right)\right\|_{\mathcal{H}^{1}},
$$

resp., in the boundary observation case,

$$
\left\|\left(u_{0}, u_{1}\right)\right\|_{\mathcal{H}^{0}} \leq C \mathrm{e}^{\kappa \mu}\left\|\partial_{\nu} u\right\|_{L^{2}((-T, T) \times \Gamma)}+\frac{C}{\mu}\left\|\left(u_{0}, u_{1}\right)\right\|_{\mathcal{H}^{1}},
$$

with $C=C_{0} \mathrm{e}^{C_{0} \sqrt{\|c\|_{L \infty}}}$.

These estimates will eventually lead to the general bound of the form $\mathfrak{C}_{o b s}=C \exp \left(\exp \left(C\|c\|_{L^{\infty}(M)}^{1 / 2}\right)\right)$. This result is a direct consequence of the following energy estimates.

Lemma 3.3. There exists $C>0$ such for any u solution of (1.13), we have

$$
\begin{gathered}
\left\|\left(u(t), \partial_{t} u(t)\right)\right\|_{\mathcal{H}^{1}} \leq C \mathrm{e}^{C|t-s| \sqrt{\|c\|_{L^{\infty}}}}\left\|\left(u(s), \partial_{t} u(s)\right)\right\|_{\mathcal{H}^{1}}, \\
\left\|\left(u(t), \partial_{t} u(t)\right)\right\|_{\mathcal{H}^{0}} \leq C \mathrm{e}^{C|t-s| \sqrt{\|c\|_{L^{\infty}}}}\left\|\left(u(s), \partial_{t} u(s)\right)\right\|_{\mathcal{H}^{0}} .
\end{gathered}
$$

For any $T>0$ there exists $C>0$ such that for any $u$ solution of (1.13), we have

$$
\begin{aligned}
& C^{-1} \mathrm{e}^{-C \sqrt{\|c\|_{L^{\infty}}}}\|u\|_{H^{1}((-T, T) \times M)} \leq\left\|\left(u_{0}, u_{1}\right)\right\|_{\mathcal{H}^{1}} \leq C \mathrm{e}^{C \sqrt{\|c\|_{L^{\infty}}}}\|u\|_{H^{1}((-T, T) \times M)}, \\
& C^{-1} \mathrm{e}^{-C \sqrt{\|c\|_{L^{\infty}}}}\|u\|_{L^{2}((-T, T) \times M)} \leq\left\|\left(u_{0}, u_{1}\right)\right\|_{\mathcal{H}^{0}} \leq C \mathrm{e}^{C \sqrt{\|c\|_{L^{\infty}}}}\|u\|_{L^{2}((-T, T) \times M)} .
\end{aligned}
$$


The nontrivial part of this Lemma is in the power $1 / 2$ for the size of the potential. Estimates (3.1) and (3.2) are proved in [13] using a modified energy method (see estimate (2.50) and (2.44) in that reference, see also [53]). Both estimates in (3.3) and the first part of (3.4) are obtained by integration on $(-T, T)$. The second estimate of (3.4) is obtained from (3.3) by a duality argument (see the proof of Theorem 6.1 in [23]). A similar argument will be performed in the proof of Lemma 3.5.

In the case when $c$ belongs to $L_{\delta}^{\infty}$, the exponential dependence with respect to $c$ in the constant $\mathfrak{C}_{o b s}$ can in fact be improved. We stress the fact that potentials in $L_{\delta}^{\infty}$ are real-valued so that $-\Delta+c$ is selfadjoint on $L^{2}$. If $c \in L_{0}^{\infty}$, the operator $-\Delta+c$ is nonnegative.

Setting

$$
\mathscr{E}_{c}\left(u, \partial_{t} u\right)=\frac{1}{2}\left(\left\|\partial_{t} u\right\|_{L^{2}(M)}^{2}+\|\nabla u\|_{L^{2}(M)}^{2}+\int_{M} c|u|^{2}\right),
$$

we always have

$$
\mathscr{E}_{c}\left(u, \partial_{t} u\right) \leq\left(1+\|c\|_{L^{\infty}(M)}\right) \mathscr{E}_{1}\left(u, \partial_{t} u\right)
$$

and, if $c \in L_{\delta}^{\infty}$ with $\delta>0$ we also obtain

$$
\begin{aligned}
\mathscr{E}_{1}\left(u, \partial_{t} u\right) & =\frac{1}{2}\left(\left\|\partial_{t} u\right\|_{L^{2}(M)}^{2}+\|\nabla u\|_{L^{2}(M)}^{2}+\|u\|_{L^{2}(M)}^{2}\right) \\
& \leq \frac{1}{2}\left(\left\|\partial_{t} u\right\|_{L^{2}(M)}^{2}+\|\nabla u\|_{L^{2}(M)}^{2}+\delta^{-1}\left(\int_{M}|\nabla u|^{2}+c|u|^{2}\right)\right) \\
& \leq\left(1+\delta^{-1}\right) \mathscr{E}_{c}\left(u, \partial_{t} u\right) .
\end{aligned}
$$

We have the following elementary Lemma which applies for any $c \in L_{\delta}^{\infty} \subset L_{0}^{\infty}, \delta \geq 0$.

Lemma 3.4. Let $T>0$. Then, there exists $C_{T}>0$ such that for all $c \in L_{0}^{\infty}$, all $\left(u_{0}, u_{1}\right) \in H_{0}^{1}(M) \times L^{2}(M)$, $g \in L^{1}\left(0, T ; L^{2}(M)\right)$ and $u$ associated solution of

$$
\left\{\begin{aligned}
\partial_{t}^{2} u-\Delta u+c u & =g, \\
u_{\mid \partial M} & =0, \quad \text { if } \partial M \neq \emptyset \\
\left(u, \partial_{t} u\right)_{t=0} & =\left(u_{0}, u_{1}\right),
\end{aligned}\right.
$$

we have the estimate

$$
\sup _{t \in[0, T]}\left(\mathscr{E}_{c}\left(u, \partial_{t} u\right)\right) \leq C_{T}\left(\mathscr{E}_{c}\left(u_{0}, u_{1}\right)+\|g\|_{L^{1}\left(0, T ; L^{2}(M)\right.}^{2}\right)
$$

If moreover $g=0$, then we have $\mathscr{E}_{C}\left(u, \partial_{t} u\right)=\mathscr{E}_{c}\left(u_{0}, u_{1}\right)$ on $(0, T)$.

Proof. Note first that $c \in L_{0}^{\infty}$ ensures that $\mathscr{E}_{c}$ is nonnegative. Multiply the equation by $\partial_{t} \bar{u}$, take real part and integrate on $M$ to obtain (at least for smooth solutions)

$$
\begin{aligned}
\frac{\mathrm{d}}{\mathrm{d} t}\left(\mathscr{E}_{C}\left(u, \partial_{t} u\right)\right) & =\int_{M} g(t, x) \partial_{t} \bar{u}(t, x) \leq\|g(t)\|_{L^{2}(M)}\left\|\partial_{t} u(t)\right\|_{L^{2}(M)} \\
& \leq\|g(t)\|_{L^{2}(M)} \sqrt{2 \mathscr{E}_{c}\left(u, \partial_{t} u\right)}
\end{aligned}
$$

An appropriate Gronwall inequality gives the expected estimate. The case $g=0$ comes from the first identity.

Now, we prove the following bound by duality. 
Lemma 3.5. For all $T, \varepsilon>0$ there is $C_{\varepsilon, T}>0$ such that for all $\delta>0, c \in L_{\delta}^{\infty}$, all $\left(u_{0}, u_{1}\right) \in H_{0}^{1}(M) \times L^{2}(M)$, and all associated solution $u \in C^{0}\left(0, T ; H_{0}^{1}(M)\right) \cap C^{1}\left(0, T ; L^{2}(M)\right)$ of $(3.6)$ with $g=0$, we have,

$$
\|u\|_{L^{2}((-T, T) \times M)} \leq\left(1+\delta^{-1}\right)^{\frac{1}{2}} C_{\varepsilon, T}\|u\|_{L^{2}((-\varepsilon, \varepsilon) \times M)} .
$$

Proof. Define $v$ to be the unique (backward) solution to

$$
\left\{\begin{aligned}
\left(\partial_{t}^{2}-\Delta+c\right) v & =u \\
v_{\mid \partial M} & =0 \\
\left(v, \partial_{t} v\right)_{\mid t=T} & =(0,0)
\end{aligned}\right.
$$

By integration by parts, we have

$$
\int_{0}^{T} \int_{M}|u|^{2}=\int_{0}^{T} \int_{M} u\left(\partial_{t}^{2}-\Delta+c\right) \bar{v}=\int_{M} \partial_{t} u(0) \bar{v}(0)-\int_{M} u(0) \partial_{t} \bar{v}(0) .
$$

But now, take $\chi \in C^{\infty}([0, T])$ with $\chi=1$ close to 0 and $\chi=0$ for $t \in[\varepsilon, T]$. Define $w=\chi(t) v$ solution of

$$
\left\{\begin{aligned}
\left(\partial_{t}^{2}-\Delta+c\right) w & =\chi u+2 \dot{\chi}(t) \partial_{t} v+\ddot{\chi}(t) v=: g=: g_{1}+g_{2} \\
w_{\mid \partial M} & =0 \\
\left(w, \partial_{t} w\right)_{\mid t=0} & =\left(v, \partial_{t} v\right)_{\mid t=0}, \\
\left(w, \partial_{t} w\right)_{\mid t=T} & =(0,0) .
\end{aligned}\right.
$$

with $g_{1}=\chi u$. We have the estimate

$$
\left\|g_{2}\right\|_{L^{2}((0, T) \times M)}^{2} \leq C\left\|\left(v, \partial_{t} v\right)\right\|_{L^{2}\left([0, T], \mathcal{H}^{1}(M)\right)}^{2}=2 C \int_{0}^{T} \mathscr{E}_{1}\left(v, \partial_{t} v\right) \mathrm{d} t .
$$

Moreover, (3.5) then yields $\mathscr{E}_{1}\left(v, \partial_{t} v\right) \leq C\left(1+\delta^{-1}\right) \mathscr{E}_{c}\left(v, \partial_{t} v\right)$ so that

$$
\left\|g_{2}\right\|_{L^{2}((0, T) \times M)}^{2} \leq C \int_{0}^{T} \mathscr{E}_{c}\left(v, \partial_{t} v\right) \mathrm{d} t \leq C T\left(1+\delta^{-1}\right) \sup _{t \in[0, T]} \mathscr{E}_{c}\left(v, \partial_{t} v\right)(t) .
$$

Then, the equation satisfied by $v$ together with Lemma 3.4 give

$$
\left\|g_{2}\right\|_{L^{2}((0, T) \times M)}^{2} \leq C T\left(1+\delta^{-1}\right) \sup _{t \in[0, T]} \mathscr{E}_{C}\left(v, \partial_{t} v\right)(t) \leq C_{T}\left(1+\delta^{-1}\right)\|u\|_{L^{2}((0, T) \times M)}^{2} .
$$

Since $g_{1}=\chi u$ trivially satisfies this estimate, we finally obtain, with $g=g_{1}+g_{2}$ (we drop the dependence with respect to $T$ or $\varepsilon$ )

$$
\|g\|_{L^{2}((0, T) \times M)}^{2} \leq C\left(1+\delta^{-1}\right)\|u\|_{L^{2}((0, T) \times M)}^{2} .
$$

The same computation as in (3.7) for $w$, noticing that the boundary value of $w$ are the same as $v$, yields the identity

$$
\int_{0}^{T} \int_{M} u \bar{g}=\int_{0}^{T} \int_{M} u\left(\partial_{t}^{2}-\Delta+c\right) \bar{w}=\int_{M} \partial_{t} u(0) \bar{v}(0)-\int_{M} u(0) \partial_{t} \bar{v}(0) .
$$

Identifying this right hand-side with that of (3.7), we therefore obtain

$$
\int_{0}^{T} \int_{M} u \bar{g}=\int_{0}^{T} \int_{M}|u|^{2}
$$


Moreover, since $g$ is supported in $[0, \varepsilon]$, and using (3.8) we have

$$
\begin{aligned}
\int_{0}^{T} \int_{M}|u|^{2} & =\int_{0}^{\varepsilon} \int_{M} u \bar{g} \leq\|u\|_{L^{2}((0, \varepsilon) \times M)}\|g\|_{L^{2}((0, \varepsilon) \times M)} \\
& \leq C\left(1+\delta^{-1}\right)^{\frac{1}{2}}\|u\|_{L^{2}([0, \varepsilon] \times M)}\|u\|_{L^{2}((0, T) \times M)} .
\end{aligned}
$$

and therefore $\|u\|_{L^{2}((0, T) \times M)} \leq C\left(1+\delta^{-1}\right)^{\frac{1}{2}}\|u\|_{L^{2}((0, \varepsilon) \times M)}$. Changing $u(t)$ into $u(-t)$ also leads to $\|u\|_{L^{2}((-T, 0) \times M)} \leq C\left(1+\delta^{-1}\right)^{\frac{1}{2}}\|u\|_{L^{2}((-\varepsilon, 0) \times M)}$, which concludes the proof of the lemma.

With this refined energy estimates (with respect to those of the proof of [23], Thm. 6.2) and using the quantitative unique continuation result of Theorem 3.1 above, we can then prove the following result.

Corollary 3.6. Let $T>\mathcal{L}(M, \omega)$ (resp.T $>\mathcal{L}(M, \Gamma)$ ). There exist $C, \kappa, \mu_{0}>0$ such that for any $\left(u_{0}, u_{1}\right) \in$ $H_{0}^{1}(M) \times L^{2}(M)$, for any $\delta>0$, any $c \in L_{\delta}^{\infty}$, and associated solution $u$ of $(1.13)$, we have, with $C_{\delta}=$ $C\left(1+\delta^{-1 / 2}\right)$, the estimate

$$
\|u\|_{L^{2}((-T, T) \times M)} \leq C_{\delta} \mathrm{e}^{\kappa \mu}\|u\|_{L^{2}\left((-T, T) ; H^{1}\left(\omega_{0}\right)\right)}+\frac{C_{\delta}}{\mu}\left(\left\|\left(u_{0}, u_{1}\right)\right\|_{\mathcal{H}^{1}(M)}+\|c u\|_{L^{2}((-T, T) \times M)}\right) .
$$

resp., the estimate

$$
\|u\|_{L^{2}((-T, T) \times M)} \leq C_{\delta} \mathrm{e}^{\kappa \mu}\left\|\partial_{\nu} u\right\|_{L^{2}((-T, T) \times \Gamma)}+\frac{C_{\delta}}{\mu}\left(\left\|\left(u_{0}, u_{1}\right)\right\|_{\mathcal{H}^{1}(M)}+\|c u\|_{L^{2}((-T, T) \times M)}\right) .
$$

for all $\mu \geq \mu_{0} \max \left\{1,\|c\|_{L^{\infty}}^{\frac{2}{3}}\right\}$.

Proof. We start again from the above Theorem 3.1, namely, for all $\mu \geq \mu_{0} \max \left\{1,\|c\|_{L^{\infty}}^{\frac{2}{3}}\right\}$, we have

$$
\|u\|_{L^{2}((-\varepsilon, \varepsilon) \times M)} \leq C \mathrm{e}^{\kappa \mu}\|u\|_{L^{2}\left((-T, T) ; H^{1}(\omega)\right)}+\frac{C}{\mu}\|u\|_{H^{1}((-T, T) \times M)} .
$$

Lemma 3.5 then gives

$$
\|u\|_{L^{2}((-T, T) \times M)} \leq C_{\delta} \mathrm{e}^{\kappa \mu}\|u\|_{L^{2}\left((-T, T) ; H^{1}(\omega)\right)}+\frac{C_{\delta}}{\mu}\|u\|_{H^{1}((-T, T) \times M)} .
$$

Then, using classical hyperbolic energy estimates, viewing $c u$ as a source term, we have

$$
\|u\|_{H^{1}((-T, T) \times M)} \leq C\left(\left\|\left(u_{0}, u_{1}\right)\right\|_{\mathcal{H}^{1}(M)}+\|c u\|_{L^{2}((-T, T) \times M)}\right) .
$$

Plugging this last estimate into the previous one yields the sought result.

\subsection{The full observability estimate}

We now combine the quantitative unique continuation result of Corollary 3.2 (general case) or 3.6 (case $c \in L_{\delta}^{\infty}$ ) with an observability estimate (or a relaxed observability estimate) for the wave equation without potential (used here as a black box) to prove Theorem 1.5. The following is e.g. given in $[1,2]$.

Theorem 3.7 ([2]). Assumes that $(\omega, T)$ satisfies $G C C$, resp.that $(\Gamma, T)$ satisfies $G C C_{\partial}$. Then, there exist $C_{0}, C_{1}>0$ such that for any $\left(w_{0}, w_{1}\right) \in H_{0}^{1}(M) \times L^{2}(M)$, and associated solution $w$ of

$$
\left\{\begin{array}{l}
\partial_{t}^{2} w-\Delta w=0 \\
\left.w\right|_{\partial M}=0, \quad \text { if } \partial M \neq \emptyset, \\
\left(w(0), \partial_{t} w(0)\right)=\left(w_{0}, w_{1}\right),
\end{array}\right.
$$


we have

$$
\int_{0}^{T}\|w(t)\|_{H^{1}(\omega)}^{2} \mathrm{~d} t \geq C_{1} \mathscr{E}_{1}\left(w_{0}, w_{1}\right)
$$

resp.,

$$
\int_{0}^{T}\left\|\partial_{\nu} w(t)\right\|_{L^{2}(\Gamma)}^{2} \mathrm{~d} t \geq C_{1} \mathscr{E}_{1}\left(w_{0}, w_{1}\right)
$$

Remark 3.8. Note that we only need (3.12) and (3.13) under the relaxed form (1.4) (i.e. with a remainder term of the form $\left.C \mathscr{E}_{0}\left(w_{0}, w_{1}\right)\right)$. Here, it is stated as in $[1,2]$.

Proof of Theorem 1.5. Both estimates (boundary and internal observation) are proved the same way, so we only detail e.g. the internal case. We shall however stress when the proof of the boundary observation differs.

With $w$ solution of (3.11) and $v$ solution of (1.13), starting from the same initial data $V_{0}=\left(v_{0}, v_{1}\right)=\left(w_{0}, w_{1}\right)$, we have, setting $z=w-v$,

$$
\left\{\begin{array}{l}
\partial_{t}^{2} z-\Delta z=c(x) v, \\
\left.z\right|_{\partial M}=0, \quad \text { if } \partial M \neq \emptyset \\
\left.\left(z, \partial_{t} z\right)\right|_{t=0}=(0,0) .
\end{array}\right.
$$

Then, the hyperbolic energy estimates for $z$ yield

$$
\int_{0}^{T}\|z(t)\|_{H^{1}(\omega)}^{2} \mathrm{~d} t \leq C\|z\|_{L^{\infty}\left(0, T ; H^{1}(M)\right)}^{2} \leq C\|c v\|_{L^{2}\left((0, T) ; L^{2}(M)\right)}^{2} .
$$

In the case of boundary observation, we use instead the so-called "hidden regularity" of the boundary normal trace for solutions to the wave equation (see for instance [28], Thm. 4.1, p. 44 in the flat case), given by

$$
\int_{0}^{T}\left\|\partial_{\nu} z(t)\right\|_{L^{2}(\Gamma)}^{2} \mathrm{~d} t \leq C\|c v\|_{L^{1}\left((0, T) ; L^{2}(M)\right)}^{2} .
$$

Hence, from the observability estimate (3.12), we obtain

$$
2 \int_{0}^{T}\|z(t)\|_{H^{1}(\omega)}^{2} \mathrm{~d} t+2 \int_{0}^{T}\|v(t)\|_{H^{1}(\omega)}^{2} \mathrm{~d} t \geq \int_{0}^{T}\|w(t)\|_{H^{1}(\omega)}^{2} \mathrm{~d} t \geq C_{1} \mathscr{E}_{1}\left(V_{0}\right),
$$

and hence

$$
\int_{0}^{T}\|v(t)\|_{H^{1}(\omega)}^{2} \mathrm{~d} t \geq C_{0} \mathscr{E}_{1}\left(V_{0}\right)-C\|c v\|_{L^{2}\left((0, T) ; L^{2}(M)\right)}^{2} .
$$

Next, in the general case $c \in L^{\infty}$, we write

$$
\|c v\|_{L^{2}\left((0, T) ; L^{2}(M)\right)}^{2} \leq\|c\|_{L^{\infty}}^{2}\|v\|_{L^{2}\left((0, T) ; L^{2}(M)\right)}^{2} \leq C \exp \left(C\|c\|_{L^{\infty}}^{\frac{1}{2}}\right) \mathscr{E}_{0}\left(V_{0}\right)
$$

according to (3.4). Note then that $(\omega, T)$ satisfies GCC implies that $T>T_{U C}(\omega)$ (resp., that $(I, T)$ satisfies $\mathrm{GCC}_{\partial}$ implies that $\left.T>T_{U C}(\Gamma)\right)$ as in the boundaryless case (see Rem. B.5). Hence, this estimate, together with (3.15) and Corollary 3.2, yields

$$
C \exp \left(C\|c\|_{L^{\infty}}^{\frac{1}{2}}\right) \mathrm{e}^{\kappa \mu} \int_{0}^{T}\|v(t)\|_{H^{1}(\omega)}^{2} \mathrm{~d} t+\frac{C \exp \left(C\|c\|_{L^{\infty}}^{\frac{1}{2}}\right)}{\mu^{2}} \mathscr{E}_{1}\left(V_{0}\right)+\int_{0}^{T}\|v(t)\|_{H^{1}(\omega)}^{2} \mathrm{~d} t \geq C_{0} \mathscr{E}_{1}\left(V_{0}\right) .
$$


for any $\mu \geq \mu_{0} \max \left\{1,\|c\|_{L^{\infty}}^{\frac{2}{3}}\right\}$. This yields the sought result in the general case $c \in L^{\infty}$, after having taken $\mu^{2} \geq \frac{2 C}{C_{0}} \exp \left(C\|c\|_{L^{\infty}}^{\frac{1}{2}}\right)$.

From now on, we consider the case $c \in L_{\delta}^{\infty}$. The strategy is slightly different. Considering $w$ the solution of (3.11) coinciding with $v$ at time $t=T / 2$ (instead of $t=0)$, we obtain similarly

$$
\int_{0}^{T}\|v(t)\|_{H^{1}(\omega)}^{2} \mathrm{~d} t \geq C_{0} \mathscr{E}_{1}\left(v, \partial_{t} v\right)(T / 2)-C\|c v\|_{L^{2}\left((0, T) ; L^{2}(M)\right)}^{2} .
$$

This uses the observability estimate (3.12) together with the fact that $w$ satisfies $\mathscr{E}_{1}\left(w, \partial_{t} w\right)(t) \leq C \mathscr{E}_{1}\left(w, \partial_{t} w\right)(0)$.

We may now use the quantitative unique continuation result of Corollary 3.6 to get rid of the term $\|c v\|_{L^{2}\left((0, T) ; L^{2}(M)\right)}^{2}$. Corollary 3.6 (applied on the time interval $(0, T)$ instead of $(-T, T)$ ) yields the existence of $C, \kappa, \mu_{0}>0$ such that for any $c \in L^{\infty}(M)$, any $v$ solution of $(1.13)$, and any $\mu \geq \mu_{0} \max \left\{1,\|c\|_{L^{\infty}}^{\frac{2}{3}}\right.$, we have, with $V(T / 2)=\left(v, \partial_{t} v\right)(T / 2)$,

$$
\begin{aligned}
\|c v\|_{L^{2}((0, T) \times M)} & \leq\|c\|_{L^{\infty}}\|v\|_{L^{2}((0, T) \times M)} \\
& \leq C_{\delta}\|c\|_{L^{\infty}} \mathrm{e}^{\kappa \mu}\|v\|_{L^{2}\left((0, T) ; H^{1}(\omega)\right)}+\frac{C_{\delta}\|c\|_{L^{\infty}}}{\mu}\left(\|V(T / 2)\|_{\mathcal{H}^{1}(M)}+\|c v\|_{L^{2}((0, T) \times M)}\right) .
\end{aligned}
$$

So, for $\mu \geq 2 C_{\delta}\|c\|_{L^{\infty}}$, we obtain

$$
\|c v\|_{L^{2}((0, T) \times M)} \leq C_{\delta}\|c\|_{L^{\infty}} \mathrm{e}^{\kappa \mu}\|v\|_{L^{2}\left((0, T) ; H^{1}(\omega)\right)}+\frac{C_{\delta}\|c\|_{L^{\infty}}}{\mu}\|V(T / 2)\|_{\mathcal{H}^{1}(M)} .
$$

Plugging this into (3.17) yields

$$
\mathscr{E}_{1}(V(T / 2)) \leq C_{\delta}^{2}\left(1+\|c\|_{L^{\infty}}\right)^{2}\left(\mathrm{e}^{2 \kappa \mu} \int_{0}^{T}\|v(t)\|_{H^{1}(\omega)}^{2} \mathrm{~d} t+\frac{1}{\mu^{2}} \mathscr{E}_{1}(V(T / 2))\right)
$$

We now take $\mu=\max \left\{\mu_{0}, \mu_{0}\|c\|_{L^{\infty}}^{\frac{2}{3}}, 2 C_{\delta}\|c\|_{L^{\infty}}, \sqrt{2} C_{\delta}\left(1+\|c\|_{L^{\infty}}\right)\right\}$ so that to absorb the last term in the right handside, and finally obtain

$$
\mathscr{E}_{1}(V(T / 2)) \leq C \mathrm{e}^{C_{\delta}\|c\|_{L^{\infty}}} \int_{0}^{T}\|v(t)\|_{H^{1}(\omega)}^{2} \mathrm{~d} t
$$

Using now e.g. (3.1) implies $\mathscr{E}_{1}(V(0)) \leq C \mathrm{e}^{\|c\|_{L}^{\frac{1}{2}} \infty} \mathscr{E}_{1}(V(T / 2))$, which concludes the proof of the theorem.

\section{Appendix A. Pseudodifferential calculus}

\section{A.1. Remainder of classical facts}

We define $S_{\mathrm{phg}}^{m}\left(T^{*} M\right)$, as the set of polyhomogeneous symbols of order $m \in \mathbb{R}$ on $M$. We recall that symbols in the class $S_{\mathrm{phg}}^{m}\left(T^{*} \mathbb{R}^{n}\right)$ behave well with respect to changes of variables, up to symbols in $S_{\mathrm{phg}}^{m-1}\left(T^{*} \mathbb{R}^{n}\right)$ (see [18], Thm. 18.1.17 and Lem. 18.1.18).

We denote by $\Psi_{\mathrm{phg}}^{m}(M)$, the space of polyhomogeneous pseudodifferential operators of order $m$ on $M$ : one says that $A \in \Psi_{\mathrm{phg}}^{m}(M)$ if

(1) its kernel $K_{A} \in \mathscr{D}^{\prime}(M \times M)$ is smooth away from the diagonal $\Delta_{M}=\{(x, x) ; x \in M\}$;

(2) for every coordinate patch $M_{\kappa} \subset M$ with coordinates $M_{\kappa} \ni x \mapsto \kappa(x) \in \tilde{M}_{\kappa} \subset \mathbb{R}^{n}$ and all $\phi_{0}, \phi_{1} \in \mathscr{C}_{c}^{\infty}\left(\tilde{M}_{\kappa}\right)$ the map

$$
u \mapsto \phi_{1}\left(\kappa^{-1}\right)^{*} A \kappa^{*}\left(\phi_{0} u\right)
$$

is in $\mathrm{Op}\left(S_{\mathrm{phg}}^{m}\left(T^{*} \mathbb{R}^{n}\right)\right)$. 
For $A \in \Psi_{\mathrm{phg}}^{m}(M)$, we denote by $\sigma_{m}(A) \in S_{\mathrm{phg}}^{m}\left(T^{*} M\right)$ the principal symbol of $A$ (see [18], Chap. 18.1). Note that the principal symbol is uniquely defined in $S_{\mathrm{phg}}^{m}\left(T^{*} M\right)$ because of the polyhomogeneous structure (see the remark following Def. 18.1.20 in [18]). Also, the map $\sigma_{m}: \Psi_{\mathrm{phg}}^{m}(M) \rightarrow S_{\mathrm{phg}}^{m}\left(T^{*} M\right)$ is onto (it suffices to construct a quantization on $T^{*} M$ by means of local charts, see for instance the discussion after Def. 18.1.20 in [18]).

In the main part of the paper, we need to consider pseudodifferential operators acting on $M$ yet depending upon the parameter $t \in(0, T)$ with some smoothness with respect to $t$. Here, we follow [11] for the definitions and notation. Let $k \in \mathbb{N} \cup\{\infty\}$, we say that $A_{t} \in \mathscr{C}^{k}\left((0, T), \operatorname{Op}\left(S_{\mathrm{phg}}^{m}\left(\mathbb{R}^{n} \times \mathbb{R}^{n}\right)\right)\right)$ if $A_{t}=\operatorname{Op}\left(a_{t}\right)$ with $a_{t} \in \mathscr{C}^{k}\left((0, T), S_{\mathrm{phg}}^{m}\left(\mathbb{R}^{n} \times \mathbb{R}^{n}\right)\right)$. Next we say that $A_{t} \in \mathscr{C}^{k}\left((0, T), \Psi_{\mathrm{phg}}^{m}(M)\right)$ if

(1) its kernel $K_{A_{t}}(x, y)$ is in $\mathscr{C}^{k}\left((0, T) ; \mathscr{C}^{\ell}\left(M \times M \backslash \Delta_{M}\right)\right)$ for all $\ell \in \mathbb{N}$;

(2) for every coordinate patch $M_{\kappa} \subset M$ with coordinates $M_{\kappa} \ni x \mapsto \kappa(x) \in \tilde{M}_{\kappa} \subset \mathbb{R}^{n}$ and all $\phi_{0}, \phi_{1} \in \mathscr{C}_{c}^{\infty}\left(\tilde{M}_{\kappa}\right)$ the map

$$
u \mapsto \phi_{1}\left(\kappa^{-1}\right)^{*} A_{t} \kappa^{*}\left(\phi_{0} u\right)
$$

is in $\mathscr{C}^{k}\left((0, T), \mathrm{Op}\left(S_{\mathrm{phg}}^{m}\left(T^{*} \mathbb{R}^{n}\right)\right)\right)$.

Let us now recall some basic facts concerning the first order hyperbolic Cauchy problem. The following result can be adapted from ([18], Chap. XXIII).

Theorem A.1. Let $\mathcal{I} \subset \mathbb{R}$ be a compact interval and take $\sigma \in \mathbb{R}$. Assume $H(t) \in \mathscr{C}^{0}\left(\mathcal{I} ; \Psi_{\mathrm{phg}}^{1}(M)\right)$ has real principal symbol. Then, there exists $C>0$ such that for all $f \in L^{1}\left(\mathcal{I} ; H^{\sigma}(M)\right)$, all $s \in \mathcal{I}$ and all $u_{0} \in H^{\sigma}(M)$, the Cauchy problem

$$
\left\{\begin{array}{l}
\partial_{t} u(t)-i H(t) u(t)=f(t), \quad t \in \mathcal{I}, \\
\left.u\right|_{t=s}=u_{0} .
\end{array}\right.
$$

has a unique (distribution) solution $u \in \mathscr{C}^{0}\left(\mathcal{I} ; H^{\sigma}(M)\right)$, that satisfies

$$
\|u\|_{L^{\infty}\left(\mathcal{I} ; H^{\sigma}(M)\right)} \leq C\left\|u_{0}\right\|_{H^{\sigma}(M)}+C\|f\|_{L^{1}\left(\mathcal{I} ; H^{\sigma}(M)\right)} .
$$

If moreover $f=0$, then $u \in \mathscr{C}^{1}\left(\mathcal{I} ; H^{\sigma-1}(M)\right)$.

The constant $C$ essentially depends on a uniform bound on $\left\|H(t)-H^{*}(t)\right\|_{L^{\infty}\left(\mathcal{I} ; \mathcal{L}\left(H^{\sigma}(M)\right)\right)}$ and commutator estimates. The fact that $C$ does not depend on the initial time $s$ follows from the proof of ([18], Lem. 23.1.1).

Note also that, in case $f=0$, the regularity $\mathscr{C}^{1}\left(\mathcal{I} ; H^{\sigma-1}(M)\right)$ of the solution $u$ implies that (A.1) is in fact an equality of functions in $\mathscr{C}^{0}\left(\mathcal{I} ; H^{\sigma-1}(M)\right)$.

As a consequence of this theorem, for all $t, s \in \mathcal{I}$, there is a bounded linear solution map $S(t, s) \in \mathcal{L}\left(H^{\sigma}(M)\right)$ (for any $\sigma \in \mathbb{R}$ ), given by $u_{0} \mapsto u(t)$, where $u$ is the unique solution to (A.1) with $f=0$. We recall that the space $\mathcal{B}\left(I ; \mathcal{L}\left(B_{1} ; B_{2}\right)\right)$ is defined in Definition 2.1. As a consequence of Theorem A.1, the solution operator $S(t, s)$ enjoys in particular the following regularity properties.

Corollary A.2. With the notations and assumptions of Theorem A.1, we have

(1) $S(t, s) \in \mathcal{B}\left(\mathcal{I} \times \mathcal{I} ; \mathcal{L}\left(H^{\sigma}(M)\right)\right)$ for all $\sigma \in \mathbb{R}$;

(2) the linear operator $\partial_{t} S(t, s): u_{0} \mapsto \partial_{t}\left(S(t, s) u_{0}\right)$ satisfies $\partial_{t} S(t, s) \in \mathcal{B}\left(\mathcal{I} \times \mathcal{I} ; \mathcal{L}\left(H^{\sigma}(M) ; H^{\sigma-1}(M)\right)\right)$ for all $\sigma \in \mathbb{R}$ together with $\partial_{t} S(t, s)-i H(t) S(t, s)=0, S(s, s)=\mathrm{Id}$;

(3) we have $S(t, s) S(s, t)=\operatorname{Id}$ for all $(s, t) \in \mathcal{I} \times \mathcal{I}$;

(4) for all $u_{0} \in H^{\sigma}(M)$ and $t \in \mathcal{I}$, the application $s \mapsto S(t, s) u_{0}$ is in $\mathscr{C}^{0}\left(\mathcal{I} ; H^{\sigma}(M)\right) \cap \mathscr{C}^{1}\left(\mathcal{I} ; H^{\sigma-1}(M)\right)$ and, defining the linear operator $\partial_{s} S(t, s): u_{0} \mapsto \partial_{s}\left(S(t, s) u_{0}\right)$, it satisfies $\partial_{s} S(t, s) \in \mathcal{B}(\mathcal{I} \times$ $\left.\mathcal{I} ; \mathcal{L}\left(H^{\sigma}(M) ; H^{\sigma-1}(M)\right)\right)$ for all $\sigma \in \mathbb{R}$ together with $\partial_{s} S(t, s)+i S(t, s) H(s)=0$. 
Points (1), (2) and (3) are direct consequences of Theorem A.1. Beware that $\partial_{t} S(t, s)$ is not a derivative in the Banach space $\mathcal{L}\left(H^{\sigma}(M) ; H^{\sigma-1}(M)\right)$. Point (4) follows from point (3) and the regularity properties of $S(t, s)$ with respect to $t$ (given in points (1) and (2)). The equation satisfied by $\partial_{s} S(t, s)$ comes from the fact that $\partial_{2} S(t, s) S(s, t)=-S(t, s) \partial_{1} S(s, t)$ (where $\partial_{1}$ and $\partial_{2}$ stand for derivatives with respect to the first and second variables respectively).

Note also that we have, for any $v \in \mathscr{C}^{0}\left(\mathcal{I} ; H^{\sigma}(M)\right) \cap \mathscr{C}^{1}\left(\mathcal{I} ; H^{\sigma-1}(M)\right)$ the formula:

$$
\partial_{t}(S(t, s) v(t))=\partial_{t} S(t, s) v(t)+S(t, s) \partial_{t} v(t) .
$$

\section{A.2. A non-autonomous non-selfadjoint Egorov theorem}

In the main part of the paper, we use the following non-selfadjoint non-autonomous version of the Egorov theorem. A semiclassical version of such a result in the autonomous case can be found in $[41,43]$.

Theorem A.3. Let $T>0$ and $H(t) \in \mathscr{C}^{\infty}\left(0, T ; \Psi_{\mathrm{phg}}^{1}(M)\right)$ having real principal symbol. Denote by $A_{1}(t):=$ $\frac{1}{2}\left(H(t)+H(t)^{*}\right) \in \mathscr{C}^{\infty}\left(0, T ; \Psi_{\mathrm{phg}}^{1}(M)\right)$ and $A_{0}(t):=\frac{1}{2 i}\left(H(t)-H(t)^{*}\right) \in \mathscr{C}^{\infty}\left(0, T ; \Psi_{\mathrm{phg}}^{0}(M)\right)$ (the adjoints being taken in $\left.L^{2}(M)\right)$, with (real-valued) principal symbols $a_{1}=\sigma_{1}\left(A_{1}\right)=\sigma_{1}(H) \in \mathscr{C}^{\infty}\left(0, T ; S_{\mathrm{phg}}^{1}\left(T^{*} M\right)\right)$, and $a_{0}=\sigma_{0}\left(A_{0}\right) \in \mathscr{C}^{\infty}\left(0, T ; S_{\mathrm{phg}}^{0}\left(T^{*} M\right)\right)$. Denote by $S(t, s)$ the solution operator associated to $\partial_{t}-i H(t)$, that is $S\left(s^{\prime}, s\right) u_{0}=u\left(s^{\prime}\right)$ where

$$
\partial_{t} u(t)-i H(t) u(t)=0,\left.\quad u\right|_{t=s}=u_{0} .
$$

Then, for any $P_{m}(s) \in \mathscr{C}^{\infty}\left((0, T), \Psi_{\mathrm{phg}}^{m}(M)\right), m \in \mathbb{R}$, there exist $Q(t, s) \in \mathscr{C}^{\infty}\left((0, T)^{2}, \Psi_{\mathrm{phg}}^{m}(M)\right)$ and

$$
\begin{array}{r}
R(t, s) \in \mathcal{B}\left((0, T)^{2}, \mathcal{L}\left(H^{\sigma}(M), H^{\sigma+1-m}(M)\right)\right) \\
\partial_{t} R(t, s), \partial_{s} R(t, s) \in \mathcal{B}\left((0, T)^{2}, \mathcal{L}\left(H^{\sigma}(M), H^{\sigma-m}(M)\right)\right)
\end{array}
$$

for all $\sigma \in \mathbb{R}$, such that we have

$$
S(s, t)^{*} P_{m}(s) S(s, t)-Q(t, s)=R(t, s), \quad(t, s) \in(0, T)^{2} .
$$

Moreover, the principal symbol of $Q(t, s)$ is given by

$$
q(t, s, \rho)=p_{m}\left(s, \chi_{s, t}(\rho)\right) \mathrm{e}^{2 \int_{s}^{t} a_{0}\left(\tau, \chi_{\tau, t}(\rho)\right) d \tau} \in \mathscr{C}^{\infty}\left((0, T)^{2}, S_{\mathrm{phg}}^{m}\left(T^{*} M\right)\right),
$$

where $p_{m}(s, \cdot)=\sigma_{m}\left(P_{m}(s)\right)$, and $\chi_{s, t}\left(\rho_{0}\right)=\rho(s, t)$ is given by the flow of the Hamiltonian vector field associated with $-a_{1}(s)$ :

$$
\frac{\mathrm{d}}{\mathrm{d} s} \rho(s, t)=H_{-a_{1}(s)}(\rho(s, t)), \quad \rho(t, t)=\rho_{0} \in T^{*} M .
$$

Note that both operators $A_{1}$ and $A_{0}$ are selfadjoint on $L^{2}(M)$ for all $t \in[0, T]$ and satisfy $H(t)=A_{1}(t)+i A_{0}(t)$. The proof is inspired from ([52], Chap. 7.8 and [41], Thm. 3.43).

Remark A.4. In this result, the error term $R(t, s)$ is 1-smoothing. Of course, a classical inductive construction (see [18], Sect. 18.1) allows to replace this by an infinitely smoothing operator. This is not needed in the present paper since we only carry an analysis at first order.

Remark A.5. In the simplest case $H=\Lambda$, we have

- $a_{1}=\lambda=|\xi|_{x}$

- $a_{0}=0$ because $\Lambda$ is selfadjoint;

- $S(t, s)=\mathrm{e}^{i(t-s) \Lambda}$ and hence $S(s, t)=\mathrm{e}^{i(s-t) \Lambda}$ and $S(s, t)^{*}=\left(\mathrm{e}^{i(s-t) \Lambda}\right)^{*}=\mathrm{e}^{i(t-s) \Lambda}$;

- $\rho(s, t)=\varphi_{(s-t)}^{-}\left(\rho_{0}\right)=\varphi_{(t-s)}^{+}\left(\rho_{0}\right)$. 
The conclusion of the Theorem (written with $s=0$ and $P_{m}$ independent on $s$ ) is therefore the classical result that $\mathrm{e}^{i t \Lambda} P_{m} \mathrm{e}^{-i t \Lambda}$ is (modulo a 1-smoothing operator) a pseudodifferential operator of order $m$ with principal symbol $q(t, \rho)=p_{m}\left(\varphi_{t}^{+}(\rho)\right)$.

Proof. First notice that $S(t, s)$ (solution operator at time $t$, issued from $s$ ) satisfies

$$
\partial_{t} S(t, s)-i H(t) S(t, s)=0, \quad S(s, s)=\mathrm{Id} .
$$

As a consequence, since $S(t, s) S(s, t)=\mathrm{Id}$, we also have, with $H(t)^{*}=A_{1}(t)-i A_{0}(t)$,

$$
\begin{aligned}
\partial_{t} S(s, t)+i S(s, t) H(t) & =0, \\
\partial_{t} S(t, s)^{*}+i S(t, s)^{*} H(t)^{*} & =0, \\
\partial_{t} S(s, t)^{*}-i H(t)^{*} S(s, t)^{*} & =0 .
\end{aligned}
$$

Corollary A.2 yields the following regularity properties

$$
S(t, s) \in \mathcal{B}\left(\mathcal{I} \times \mathcal{I} ; \mathcal{L}\left(H^{\sigma}(M)\right)\right), \quad \partial_{t} S(t, s), \partial_{s} S(t, s) \in \mathcal{B}\left(\mathcal{I} \times \mathcal{I} ; \mathcal{L}\left(H^{\sigma}(M) ; H^{\sigma-1}(M)\right)\right)
$$

as well as for $S(t, s)^{*}$, for all $\sigma \in \mathbb{R}$.

Now, setting

$$
P(t, s):=S(s, t)^{*} P_{m}(s) S(s, t),
$$

and using the above equations, we have $P(s, s)=P_{m}(s)$ with

$$
\partial_{t} P(t, s)=i H(t)^{*} P(t, s)-i P(t, s) H(t)=i\left[A_{1}(t), P(t, s)\right]+A_{0}(t) P(t, s)+P(t, s) A_{0}(t) .
$$

We now construct an approximate pseudodifferential solution $Q(t, s)$ for (A.3): its principal symbol $q(t, s, x, \xi)$ should satisfy

$$
\partial_{t} q(t, s, \cdot)=\left\{a_{1}(t, \cdot), q(t, s, \cdot)\right\}+2 a_{0}(t, \cdot) q(t, s, \cdot), \quad \text { and } \quad q(s, s, \rho)=p_{m}(s, \rho),
$$

where $\{\cdot, \cdot\}$ stands for the Poisson bracket in the $(x, \xi)$ variables.

We first check that the function $q(t, s, x, \xi)$ defined in (A.2) satisfies (A.4). From (A.2), and using $\chi_{\tau, t} \circ$ $\chi_{t, s}(\rho)=\chi_{\tau, s}(\rho)$, we have:

$$
q\left(t, s, \chi_{t, s}(\rho)\right)=p_{m}(s, \rho) \mathrm{e}^{2 \int_{s}^{t} a_{0}\left(\tau, \chi_{\tau, s}(\rho)\right) \mathrm{d} \tau} .
$$

This yields $q(s, s, \rho)=p_{m}(s, \rho)$ and

$$
\partial_{t}\left(q\left(t, s, \chi_{t, s}(\rho)\right) \mathrm{e}^{-2 \int_{s}^{t} a_{0}\left(\tau, \chi_{\tau, s}(\rho)\right) \mathrm{d} \tau}\right)=0,
$$

which, according to the definition of the flow $\chi_{t, s}$, is

$$
\left(\left(\partial_{t} q\right)(t, s, \cdot)+\left\{-a_{1}(t, \cdot), q(t, s, \cdot)\right\}-2 a_{0}(t, \cdot) q(t, s, \cdot)\right)\left(\chi_{t, s}(\rho)\right) \mathrm{e}^{-2 \int_{s}^{t} a_{0}\left(\tau, \chi_{\tau, s}(\rho)\right) \mathrm{d} \tau}=0,
$$

for all $(t, s) \in(0, T)^{2}$ and $\rho \in S^{*} M$, which proves (A.4).

Note that we use the homogeneity of $a_{1}$ of order 1 allows to keep the homogeneity of $q(t, \rho)$. This allows to select one $Q(t, s)$, so that

$$
Q(t, s) \in \mathscr{C}^{\infty}\left((0, T)^{2}, \Psi_{\mathrm{phg}}^{m}(M)\right) \text { satisfies } \sigma_{m}(Q(t, s))=q(t, s, \cdot) .
$$


From (A.4) and pseudodifferential calculus, we now have

$$
\begin{aligned}
\partial_{t} Q(t, s) & =i\left[A_{1}(t), Q(t, s)\right]+A_{0}(t) Q(t, s)+Q(t, s) A_{0}(t)+R(t, s) \\
& =i H(t)^{*} Q(t, s)-i Q(t, s) H(t)+R(t, s),
\end{aligned}
$$

with $R \in \mathscr{C}^{\infty}\left((0, T)^{2} ; \Psi_{\mathrm{phg}}^{m-1}(M)\right)$. We now estimate the remainder $Q(t, s)-P(t, s)$. We set

$$
T(t, s):=S(t, s)^{*}(Q(t, s)-P(t, s)) S(t, s)=S(t, s)^{*} Q(t, s) S(t, s)-P_{m}(s),
$$

so that we have

$$
\begin{aligned}
\partial_{t} T(t, s) & =\partial_{t}\left(S(t, s)^{*} Q(t, s) S(t, s)\right) \\
& =S(t, s)^{*}\left(-i H(t)^{*} Q(t, s)+\partial_{t} Q(t, s)+i Q(t, s) H(t)\right) S(t, s) \\
& =S(t, s)^{*} R(t, s) S(t, s),
\end{aligned}
$$

after having used (A.6). This yields

$$
Q(t, s)-P(t, s)=S(s, t)^{*}\left(Q(s, s)-P_{m}(s)+\int_{s}^{t} S\left(t^{\prime}, s\right)^{*} R\left(t^{\prime}, s\right) S\left(t^{\prime}, s\right) \mathrm{d} t^{\prime}\right) S(s, t),
$$

where $R \in \mathscr{C}^{\infty}\left((0, T)^{2} ; \Psi_{\mathrm{phg}}^{m-1}(M)\right)$ and $Q(s, s)-P_{m}(s) \in \mathscr{C}^{\infty}\left((0, T) ; \Psi_{\mathrm{phg}}^{m-1}(M)\right)$. This now implies

$$
\begin{array}{r}
Q(t, s)-P(t, s) \in \mathcal{B}\left((0, T)^{2}, \mathcal{L}\left(H^{\sigma}(M), H^{\sigma+1-m}(M)\right)\right), \\
\partial_{t}(Q(t, s)-P(t, s)), \partial_{s}(Q(t, s)-P(t, s)) \in \mathcal{B}\left((0, T)^{2}, \mathcal{L}\left(H^{\sigma}(M), H^{\sigma-m}(M)\right)\right),
\end{array}
$$

for any $\sigma \in \mathbb{R}$. This, together with the expression of $Q$ in (A.5) concludes the proof of the theorem.

\section{A.3. Smoothing properties of some operators}

The following lemma is taken from ([11], Lem. A.1) and inspired by [10].

Lemma A.6. Let $\gamma \in \mathbb{R}^{*}$ and $B_{0} \in \Psi_{\mathrm{phg}}^{0}(M)$. Then, the operator defined by

$$
B(T)=\int_{0}^{T} \mathrm{e}^{i t \gamma \Lambda} B_{0} \mathrm{e}^{i t \gamma \Lambda} \mathrm{d} t
$$

satisfies $B \in \mathcal{B}_{\text {loc }}\left(\mathbb{R} ; \mathcal{L}\left(H^{\sigma}(M), H^{\sigma+1}(M)\right)\right)$ for all $\sigma \in \mathbb{R}$.

This lemma suffices for the study of the Klein-Gordon equation in Section 2.2 (in which it is only used for $\gamma= \pm 1$ ). In the general case of Section 2.4 however, we need the following non-autonomous version of this result.

Lemma A.7. Let $\mathcal{I} \subset \mathbb{R}$ be an interval containing 0 , and let $H_{+}, H_{-} \in \mathscr{C}^{\infty}\left(\mathcal{I} ; \Psi_{\mathrm{phg}}^{1}(M)\right)$ such that $\lambda=$ $\sigma_{1}\left(H_{+}\right)=-\sigma_{1}\left(H_{-}\right) \in \mathbb{R}$ is time independant and elliptic. Then for any $B_{0} \in \Psi_{\mathrm{phg}}^{m}(M), m \in \mathbb{R}$, the operator defined by

$$
B(T)=\int_{0}^{T} S_{+}(t, 0)^{*} B_{0} S_{-}(t, 0) \mathrm{d} t
$$

where $S_{ \pm}(t, 0)$ is the solution operator for the evolution equation $\partial_{t}-i H_{ \pm}(t)$, satisfies for all $\sigma \in \mathbb{R}, B \in$ $\mathcal{B}_{\text {loc }}\left(\mathcal{I} ; \mathcal{L}\left(H^{\sigma}(M), H^{\sigma+1-m}(M)\right)\right)$.

We refer to Corollary A.2 for the properties of $S_{ \pm}(t, 0)$. We shall need the following lemma. 
Lemma A.8. Let $\mathcal{I} \subset \mathbb{R}$ be an interval containing 0 , and let $H(t) \in \mathscr{C}^{\infty}\left(\mathcal{I} ; \Psi_{\mathrm{phg}}^{1}(M)\right)$ with real principal symbol and denote by $S(t, s)$ the solution operator for the evolution equation $\partial_{t}-i H(t)$ with initial data at time s. Then, for any $A \in \Psi_{\mathrm{phg}}^{m}(M)$, we have

$$
[A, S(t, 0)]=\int_{0}^{t} S(t, s)[A, i H(s)] S(s, 0) \mathrm{d} s
$$

In particular if $A=\Lambda$ and $H(t)=\Lambda+i R(t)$, with $R \in \mathscr{C}^{\infty}\left(\mathcal{I} ; \Psi_{\mathrm{phg}}^{0}(M)\right)$, we have

$$
[\Lambda, S(t, 0)],\left[\Lambda, S(t, 0)^{*}\right] \in \mathcal{B}_{\text {loc }}\left(\mathcal{I} ; \mathcal{L}\left(H^{s}(M)\right)\right)
$$

for all $s \in \mathbb{R}$.

Proof of Lemma A.8. The function $u(t)=[A, S(t, 0)] u_{0}=A S(t, 0) u_{0}-S(t, 0) A u_{0}$ satisfies $u(0)=0$ and solves

$$
\partial_{t} u(t)=A i H(t) S(t, 0) u_{0}-i H(t) S(t, 0) A u_{0}=[A, i H(t)] S(t, 0) u_{0}+i H(t) u(t),
$$

so that the Duhamel formula directly yields (A.7).

Proof of Lemma A.7. We first notice that $B(T) \in \mathcal{B}_{\text {loc }}\left(\mathcal{I} ; \mathcal{L}\left(H^{s}(M), H^{s-m}(M)\right)\right)$ since $S_{ \pm}(t, 0)$ preserve regularity. We recall also that

$$
\partial_{t} S_{ \pm}(t, 0)-i H_{ \pm}(t) S_{ \pm}(t, 0)=0, \quad \partial_{t} S_{ \pm}(t, 0)^{*}+i S_{ \pm}(t, 0)^{*} H_{ \pm}(t)^{*}=0
$$

To prove the result, it suffices to prove that $\Lambda B(T) \in \mathcal{B}_{\text {loc }}\left(\mathcal{I} ; \mathcal{L}\left(H^{s}(M), H^{s-m}(M)\right)\right)$. We thus compute

$$
i \Lambda B(T)=\int_{0}^{T} i S_{+}(t, 0)^{*} \Lambda B_{0} S_{-}(t, 0) \mathrm{d} t+\int_{0}^{T} i\left[S_{+}(t, 0)^{*}, \Lambda\right] B_{0} S_{-}(t, 0) \mathrm{d} t .
$$

The second term belongs to $\mathcal{B}_{\text {loc }}\left(\mathcal{I} ; \mathcal{L}\left(H^{s}(M), H^{s-m}(M)\right)\right)$ according to Lemma A.8. The first term may be rewritten as

$$
\int_{0}^{T} i S_{+}(t, 0)^{*} \Lambda B_{0} S_{-}(t, 0) \mathrm{d} t=\int_{0}^{T} i S_{+}(t, 0)^{*} H_{+}(t)^{*} B_{0} S_{-}(t, 0) \mathrm{d} t+\int_{0}^{T} i\left(\Lambda-H_{+}(t)^{*}\right) S_{+}(t, 0)^{*} B_{0} S_{-}(t, 0) \mathrm{d} t .
$$

The second term belongs to $\mathcal{B}_{\text {loc }}\left(\mathcal{I} ; \mathcal{L}\left(H^{s}(M), H^{s-m}(M)\right)\right)$ since $\Lambda-H_{+}(t)^{*} \in \mathscr{C}^{\infty}\left(\mathcal{I} ; \Psi_{\text {phg }}^{0}(M)\right)$, and it remains only to examine the first one. Using (A.8), we now have, for some $R \in \mathcal{B}_{\text {loc }}\left(\mathcal{I} ; \mathcal{L}\left(H^{s}(M), H^{s-m}(M)\right)\right)$,

$$
\begin{aligned}
i \Lambda B(T) & =\int_{0}^{T}-\partial_{t} S_{+}(t, 0)^{*} B_{0} S_{-}(t, 0) \mathrm{d} t+R \\
& =\int_{0}^{T} S_{+}(t, 0)^{*} B_{0} \partial_{t} S_{-}(t, 0) \mathrm{d} t-\left[S_{+}(t, 0)^{*} B_{0} S_{-}(t, 0)\right]_{0}^{T}+R
\end{aligned}
$$

after an integration by parts (note that this is done in the weak sense, i.e. when applied to a function). Using again (A.8), we obtain, for other remainders $R \in \mathcal{B}_{\text {loc }}\left(\mathcal{I} ; \mathcal{L}\left(H^{s}(M), H^{s-m}(M)\right)\right)$,

$$
\begin{aligned}
i \Lambda B(T) & =\int_{0}^{T} S_{+}(t, 0)^{*} B_{0} i H_{-}(t) S_{-}(t, 0) \mathrm{d} t+R, \\
& =\int_{0}^{T} S_{+}(t, 0)^{*} B_{0} i(-\Lambda) S_{-}(t, 0) \mathrm{d} t+R,
\end{aligned}
$$


where we used that $-\Lambda-H_{-}(t) \in \mathscr{C}^{\infty}\left(\mathcal{I} ; \Psi_{\mathrm{phg}}^{0}(M)\right)$. Using $\left[B_{0}, \Lambda\right] \in \Psi_{\mathrm{phg}}^{m}(M)$, we now have

$$
i \Lambda B(T)=\int_{0}^{T} S_{+}(t, 0)^{*}(-i \Lambda) B_{0} S_{-}(t, 0) \mathrm{d} t+R,
$$

that is, using again Lemma A.8,

$$
i \Lambda B(T)=-i \Lambda B(T)+R,
$$

with $R \in \mathcal{B}_{\text {loc }}\left(\mathcal{I} ; \mathcal{L}\left(H^{s}(M), H^{s-m}(M)\right)\right)$. This concludes the proof of the lemma.

\section{A.4. Uniform estimates on compact manifolds}

We give here for the sake of completeness a version of the sharp Gårding inequality (and also boundedness estimates for pseudodifferential operators) on a compact manifold, with a uniform dependence of the constant w.r.t. the operator involved. Its counterpart on $\mathbb{R}^{n}$ (of which the result presented here is a consequence) is given in Theorem 2.5.4 of [27] for instance.

We use the notation $M_{\varepsilon}=\{(x, y) \in M \times M$, $\operatorname{dist}(x, y)>\varepsilon\}$.

Theorem A.9. Let $\left(U_{j}, \kappa_{j}\right)_{j=1 \ldots N}$ be a fixed atlas of $M$ and $\left(\psi_{j}\right)_{j=1 \ldots N}$ a subordinated partition of unity. Let $\tilde{\psi}_{j} \in \mathscr{C}_{c}^{\infty}\left(U_{j}\right)$ be such that $\tilde{\psi}_{j}=1$ on $\operatorname{supp}\left(\psi_{j}\right)$. Then, for all $s \in \mathbb{R}$, there exists $\gamma$ a seminorm on $S_{\mathrm{phg}}^{0}\left(T^{*} \mathbb{R}^{n}\right)$, there exist $\varepsilon>0, \ell>0$ and $C>0$ such that, for all $A \in \Psi_{\mathrm{phg}}^{0}(M)$ and all $u \in H^{s}(M)$, we have

$$
\|A u\|_{H^{s}(M)} \leq C\left(\sup _{j \in\{1 \ldots N\}} \gamma\left(a^{j}\right)+\left\|K_{A}\right\|_{W^{\ell, \infty}\left(M_{\varepsilon}\right)}\right)\|u\|_{H^{s}(M)},
$$

and, if moreover $\sigma_{0}(A) \geq 0$ on $T^{*} M$,

$$
\operatorname{Re}(A u, u)_{H^{s}(M)} \geq-C\left(\sup _{j \in\{1 \ldots N\}} \gamma\left(a^{j}\right)+\left\|K_{A}\right\|_{W^{\ell, \infty}\left(M_{\varepsilon}\right)}\right)\|u\|_{H^{s-1 / 2}(M)}^{2},
$$

where $a^{j} \in S_{\mathrm{phg}}^{0}\left(T^{*} \mathbb{R}^{n}\right)$ is the (full) symbol of the operator $\left(\kappa_{j}^{-1}\right)^{*} \psi_{j} A \tilde{\psi}_{j} \kappa_{j}^{*} \in \Psi_{\mathrm{phg}}^{0}\left(\mathbb{R}^{n}\right)$.

As a direct consequence, we have the following corollary.

Corollary A.10. Let $s \in \mathbb{R}, T_{1}<T_{2}$ and assume $A_{t} \in \mathscr{C}^{0}\left(\left[T_{1}, T_{2}\right] ; \Psi_{\mathrm{phg}}^{0}(M)\right)$. Then, there exists a constant $C>0$ such that

$$
\left\|A_{t} u\right\|_{H^{s}(M)} \leq C\|u\|_{H^{s}(M)}, \quad \text { for all } t \in\left[T_{1}, T_{2}\right], \text { and } u \in H^{s}(M),
$$

and, if moreover $\sigma_{0}(A) \geq 0$ on $\left[T_{1}, T_{2}\right] \times T^{*} M$,

$$
\operatorname{Re}\left(A_{t} u, u\right)_{H^{s}(M)} \geq-C\|u\|_{H^{s-1 / 2}(M)}^{2}, \quad \text { for all } t \in\left[T_{1}, T_{2}\right], \text { and } u \in H^{s}(M) .
$$

Proof of Theorem A.9. We only prove the uniform Gårding inequality (A.10). The proof of the uniform boundedness estimate (A.9) is the same (using e.g. [27], proof of Thm. 1.1.4).

Notice first that the result in $H^{s}(M)$ is a consequence of the result in $L^{2}(M)$ and (A.9): For $u \in \mathscr{C}^{\infty}(M)$, applying (A.10) to $\Lambda^{s} u$ yields

$$
\left(A \Lambda^{s} u, \Lambda^{s} u\right)_{L^{2}(M)} \geq-C_{0}\left\|\Lambda^{s} u\right\|_{H^{-1 / 2}(M)}^{2}=-C_{0}\|u\|_{H^{s-1 / 2}(M)}^{2},
$$

with $C_{0}=\sup _{j \in\{1 \ldots N\}} \gamma\left(a^{j}\right)+\left\|K_{A}\right\|_{W^{\ell, \infty}\left(M_{\varepsilon}\right)}$. Writing now

$$
\left|\left(A \Lambda^{s} u, \Lambda^{s} u\right)_{L^{2}(M)}-\left(\Lambda^{s} A u, \Lambda^{s} u\right)_{L^{2}(M)}\right|=\left|\left(\Lambda^{1 / 2}\left[A, \Lambda^{s}\right] u, \Lambda^{s-1 / 2} u\right)_{L^{2}(M)}\right| \leq C_{A}\|u\|_{H^{s-1 / 2}(M)}^{2},
$$

where the constant $C_{A}$ has the same form as $C_{0}$ according to (A.9), yields the result in $H^{s}(M)$. 
Let us now prove the case $s=0$. We have

$$
A=\sum_{j=1 \ldots N} \psi_{j} A=\sum_{j=1 \ldots N} \psi_{j} A \tilde{\psi}_{j}+\psi_{j} A\left(1-\tilde{\psi}_{j}\right) .
$$

The kernel of each operator $\psi_{j} A\left(1-\tilde{\psi}_{j}\right)$ is given by $K^{j}(x, y):=\psi_{j}(x) K_{A}(x, y)\left(1-\tilde{\psi}_{j}(y)\right)$. Since $\psi_{j}\left(1-\tilde{\psi}_{j}\right)=0$, it is supported in the set $M_{\varepsilon^{j}}$ for some $\varepsilon^{j}>0$. As a consequence, this operator is infinitely smoothing and we have in particular

$$
\left\|\psi_{j} A\left(1-\tilde{\psi}_{j}\right)\right\|_{\mathcal{L}\left(H^{-1 / 2}(M) ; H^{1 / 2}(M)\right)} \leq C_{j}\left\|K_{A}\right\|_{W^{\ell, \infty}\left(M_{\varepsilon} j\right)},
$$

so that

$$
\begin{aligned}
\left|\left(\psi_{j} A\left(1-\tilde{\psi}_{j}\right) u, u\right)_{L^{2}(M)}\right| & \leq \|\left(\psi_{j} A\left(1-\tilde{\psi}_{j}\right) u\left\|_{H^{1 / 2}(M)}\right\| u \|_{H^{-1 / 2}(M)}\right. \\
& \leq C_{j}\left\|K_{A}\right\|_{W^{\ell, \infty}\left(M_{\varepsilon}\right)}\|u\|_{H^{-1 / 2}(M)}^{2} .
\end{aligned}
$$

Next, concerning the terms of the form $\psi_{j} A \tilde{\psi}_{j}$ in (A.11), we write

$$
\left(\psi_{j} A \tilde{\psi}_{j} u, u\right)_{L^{2}(M)}=\left(\left(\left(\kappa_{j}^{-1}\right)^{*} \psi_{j} A \tilde{\psi}_{j} \kappa_{j}^{*}\right)\left(\kappa_{j}^{-1}\right)^{*} u,\left(\kappa_{j}^{-1}\right)^{*} u\right)_{L^{2}\left(\mathbb{R}^{n}, \sqrt{\operatorname{det}(g)} \mathrm{d} L\right)},
$$

where the principal symbol of the operator $\left(\kappa_{j}^{-1}\right)^{*} \psi_{j} A \tilde{\psi}_{j} \kappa_{j}^{*}$ is $\left(\kappa_{j}^{-1}\right)^{*}\left(\psi_{j} \sigma_{0}(a)\right) \geq 0$ on $T^{*} \mathbb{R}^{n}$. Using the sharp Gårding inequality in $\mathbb{R}^{n}$ as stated in ([27], Thm. 2.5.4), we obtain, for smooth compactly supported functions $v$,

$$
\left(\left(\left(\kappa_{j}^{-1}\right)^{*} \psi_{j} A \tilde{\psi}_{j} \kappa_{j}^{*}\right) v, v\right)_{L^{2}\left(\mathbb{R}^{n}, \sqrt{\operatorname{det}(g)} \mathrm{d} L\right)} \geq-C_{j} \gamma\left(a^{j}\right)\|v\|_{H^{-1 / 2}\left(\mathbb{R}^{n}\right)}^{2} .
$$

Note that we have used here that the sharp Gårding inequality remains unchanged under the addition of an operator in $\Psi_{\mathrm{phg}}^{-1}\left(\mathbb{R}^{n}\right)$.

Finally, combining (A.12), (A.13), with (A.11), and recalling that there is a finite number of coordinate patches $U_{j}$, we obtain the result of Theorem A.9.

\section{Appendix B. Geometric facts}

\section{B.1. Definitions and notations}

Recall that $M$ is a compact manifold without boundary, that for $x \in M, T_{x} M$ denotes the tangent space to $M$ at the point $x$, and $T_{x}^{*} M$ its dual space, the cotangent space to $M$ at $x$. We also denote $\pi: T M \rightarrow M$ and $\pi: T^{*} M \rightarrow M$ the canonical projections to the manifold, the duality bracket at $x$ being denoted by $\langle\cdot, \cdot\rangle_{x}=\langle\cdot, \cdot\rangle_{T_{x}^{*} M, T_{x} M}$. The manifold $M$ is endowed with a Riemannian metric $g$, that is: for any $x \in M, g_{x}$ is a positive definite quadratic form on $T_{x} M$, depending smoothly on $x$. The Riemannian metric $g$ furnishes an isomorphism $T_{x} M \rightarrow T_{x}^{*} M, v \mapsto v^{b}:=g_{x}(v, \cdot)$, with inverse $v=\left(v^{b}\right)^{\sharp}$. The metric $g$ on $T M$ induces a metric $g^{*}$ on $T^{*} M$, canonically defined by $g_{x}^{*}(\xi, \eta)=g_{x}\left(\xi^{\sharp}, \eta^{\sharp}\right)$ for $x \in M$, and $\xi, \eta \in T_{x}^{*} M$. We denote by $S M$ (resp. $\left.S^{*} M\right)$ the Riemannian sphere (resp.cosphere) bundle over $M$, with fiber over $x \in M$ given by $\left\{v \in T M, g_{x}(v, v)=1\right\}$ (resp. $\left.\left\{\xi \in T^{*} M, g_{x}^{*}(\xi, \xi)=1\right\}\right)$.

We define the Hamiltonian $\lambda(x, \xi)=|\xi|_{x}=\sqrt{g_{x}^{*}(\xi, \xi)} \in \mathscr{C}^{\infty}\left(T^{*} M \backslash 0\right)$, which is a homogeneous function of degree one in the variable $\xi \in T_{x}^{*} M$. We denote by $H_{\lambda}$ and $\varphi_{t}=\varphi_{t}^{+}$the associated Hamiltonian vector field and flow, that is

$$
\frac{\mathrm{d}}{\mathrm{d} t} \varphi_{t}(\rho)=H_{\lambda}\left(\varphi_{t}(\rho)\right), \quad \varphi_{0}(\rho)=\rho \in T^{*} M
$$

with, in local charts, $H_{\lambda}=\partial_{\xi} \lambda \cdot \partial_{x}-\partial_{x} \lambda \cdot \partial_{\xi}$. Writing $\varphi_{t}(\rho)=(x(t), \xi(t))$, we have, still in local charts,

$$
\dot{x}(t)=\partial_{\xi} \lambda(x(t), \xi(t)), \quad \text { and } \quad \dot{\xi}(t)=-\partial_{x} \lambda(x(t), \xi(t)) .
$$


This flow is globally defined, for it preserves the function $\lambda$. In particular $(x(t), \xi(t)) \in S^{*} M=\{(x, \xi) \in$ $\left.T^{*} M, \lambda(x, \xi)=1\right\}$ for all $t \in \mathbb{R}$ if $(x(0), \xi(0)) \in S^{*} M$. The following lemma gives the link between geodesics and projections on $M$ of the curves of $\varphi_{t}$ (see for instance [15], Thm. 2.124 in the case of $H_{\lambda^{2} / 2}=\lambda H_{\lambda}$, which gives the same result up to a reparametrization of the curve $x(t)$ ).

Lemma B.1. Let $I=[a, b] \subset \mathbb{R}$. A curve $(x(t), \xi(t))_{t \in I}$ on $T^{*} M \backslash 0$ is a Hamiltonian curve of $\lambda$ (i.e. satisfies (B.1)) in $T^{*} M \backslash 0$ if and only if the curve $(x(t))_{t \in I}$ on $M$ is a geodesic curve of the metric $g$ on $M$ (parametrized by arclength) such that $(x(t), \dot{x}(t)) \in S M, t \in I$. Moreover, we have $\dot{x}(t)=\frac{\xi(t)^{\sharp}}{|\xi(t)|_{x(t)}} \in S_{x(t)} M$, $t \in I$.

In the main part of the article, we also use the Hamiltonian flow $\varphi_{t}^{-}$associated to the Hamiltonian $-\lambda$ (which, as well, is global and preserves $\left.S^{*} M\right)$. Of course, it is linked with that of $\lambda$ according to the following lemma.

Lemma B.2. For all $t \in \mathbb{R}$ and $\rho \in T^{*} M$, we have $\varphi_{t}^{-}(\rho)=\varphi_{-t}(\rho)$. Moreover, denoting by $\sigma: T^{*} M \rightarrow T^{*} M$ the involution $(x, \xi) \mapsto(x,-\xi)$, we have $\sigma \circ \varphi_{t}(\rho)=\varphi_{-t} \circ \sigma(\rho)$.

This is classical. The first fact is e.g. a consequence of ([11], Lem. B.1), and the second of ([11], Lem. B.3).

In the main part of the paper, we use the Riemannian distance to a subset $E \subset M$, defined by

$$
\operatorname{dist}\left(x_{1}, E\right)=\inf _{x_{0} \in E} \operatorname{dist}\left(x_{0}, x_{1}\right)
$$

with

$$
\operatorname{dist}\left(x_{0}, x_{1}\right)=\inf _{\gamma \in C^{1}([0,1] ; M), \gamma(0)=x_{0}, \gamma(1)=x_{1}} \operatorname{length}(\gamma),
$$

where the Riemannian length of a path $\gamma \in C^{1}([0,1] ; M)$ is given by length $(\gamma)=\int_{0}^{1} \sqrt{g_{\gamma(t)}(\dot{\gamma}(t), \dot{\gamma}(t))} \mathrm{d} t$.

Given a smooth function $u$ on $M$, we define the vector field $\nabla u$ by $\nabla u(x)=(d u(x))^{\sharp}$. As well, the LaplaceBeltrami operator may be defined by the identity

$$
\int_{M}(\Delta u)(x) v(x) \mathrm{d} x=-\int_{M} g_{x}(\nabla u(x), \nabla v(x)) \mathrm{d} x
$$

where $\mathrm{d} x$ is the Riemannian volume element (given by $\sqrt{\operatorname{det}(g)} d L(x)$ in local charts, where $d L(x)$ is the Lebesgue measure).

As a corollary of Lemma B.2, we may now give a Proof of Lemma 2.15.

Proof of Lemma 2.15. Recalling that $\varphi_{s}^{-}(\rho)=\varphi_{-s}^{+}(\rho)=\varphi_{-s}(\rho)$ according to Lemma B.2, (2.49) can be rewritten, using $(x(s), \xi(s))=\varphi_{s}\left(x_{0}, \xi_{0}\right)$ for $s \in \mathbb{R}$ as

$$
g_{T}^{ \pm}\left(x_{0}, \xi_{0}\right)=\int_{0}^{T} b_{\omega}^{2}(x( \pm t)) \exp \left(\int_{0}^{t} \operatorname{Re}\left(b_{0}\right)(\tau, x( \pm \tau)) \pm\left\langle\frac{\xi( \pm \tau)}{|\xi( \pm \tau)|_{x( \pm \tau)}}, \operatorname{Re}\left(b_{1}\right)(\tau, x( \pm \tau))\right\rangle_{x( \pm \tau)} \mathrm{d} \tau\right) \mathrm{d} t .
$$

According to Lemma B.2, we also have $\sigma \circ \varphi_{s}=\varphi_{-s} \circ \sigma$ (where $\sigma(x, \xi)=(x,-\xi)$ ), that is, denoting $\left(x\left(s, x_{0}, \xi_{0}\right), \xi\left(s, x_{0}, \xi_{0}\right)\right)=\varphi_{s}\left(x_{0}, \xi_{0}\right)$,

$$
\left.\left.x\left(-s, x_{0},-\xi_{0}\right)=x\left(s, x_{0}, \xi_{0}\right)\right), \quad \xi\left(-s, x_{0},-\xi_{0}\right)=-\xi\left(s, x_{0}, \xi_{0}\right)\right), \quad s \in \mathbb{R},\left(x_{0}, \xi_{0}\right) \in T^{*} M .
$$


Plugging this into the expression of $g_{T}^{-}$, we obtain

$$
\begin{aligned}
g_{T}^{-}\left(x_{0},-\xi_{0}\right)= & \int_{0}^{T} b_{\omega}^{2}\left(x\left(-t, x_{0},-\xi_{0}\right)\right) \exp \left(\int_{0}^{t} \operatorname{Re}\left(b_{0}\right)\left(\tau, x\left(-\tau, x_{0},-\xi_{0}\right)\right)\right. \\
& \left.-\left\langle\frac{\xi\left(-\tau, x_{0},-\xi_{0}\right)}{\left|\xi\left(-\tau, x_{0},-\xi_{0}\right)\right|_{x\left(-\tau, x_{0},-\xi_{0}\right)}}, \operatorname{Re}\left(b_{1}\right)\left(\tau, x\left(-\tau, x_{0},-\xi_{0}\right)\right)\right\rangle_{x\left(-\tau, x_{0},-\xi_{0}\right)} \mathrm{d} \tau\right) \mathrm{d} t \\
= & \int_{0}^{T} b_{\omega}^{2}\left(x\left(t, x_{0}, \xi_{0}\right)\right) \exp \left(\int_{0}^{t} \operatorname{Re}\left(b_{0}\right)\left(\tau, x\left(\tau, x_{0}, \xi_{0}\right)\right)\right. \\
& \left.-\left\langle\frac{-\xi\left(\tau, x_{0}, \xi_{0}\right)}{\left|\xi\left(\tau, x_{0}, \xi_{0}\right)\right|_{x\left(\tau, x_{0}, \xi_{0}\right)}}, \operatorname{Re}\left(b_{1}\right)\left(\tau, x\left(\tau, x_{0}, \xi_{0}\right)\right)\right\rangle_{x\left(\tau, x_{0}, \xi_{0}\right)} \mathrm{d} \tau\right) \mathrm{d} t \\
= & g_{T}^{+}\left(x_{0}, \xi_{0}\right) .
\end{aligned}
$$

This is $g_{T}^{-} \circ \sigma=g_{T}^{+}$.

Note that Lemma 2.15 contains in particular the following result.

Corollary B.3. For any function $f \in \mathscr{C}^{0}(M)$, for any $T>0$, we have

$$
\min _{\rho \in S^{*} M} \int_{0}^{T} f \circ \pi \circ \varphi_{t}^{+}(\rho) \mathrm{d} t=\min _{\rho \in S^{*} M} \int_{0}^{T} f \circ \pi \circ \varphi_{t}^{-}(\rho) \mathrm{d} t .
$$

\section{B.2. Comparing $T_{U C}(\omega)$ and $T_{G C C}(\omega)$}

In this section, we briefly prove that $2 \mathcal{L}(M, \omega)=T_{U C}(\omega) \leq T_{G C C}(\omega)$ (where these quantities are defined in (1.5), (1.6) and (1.7) respectively) in general and study the case of equality.

Lemma B.4. We always have $T_{G C C}(E) \geq 2 \mathcal{L}(M, E)$.

Proof. Let $\varepsilon>0$, we prove that for any $x \in M, 2 \operatorname{dist}(x, E) \leq T_{G C C}(E)+2 \varepsilon$.

Fix $x \in M$. By definition, there exists $x_{1} \in E$ so that

$$
\operatorname{dist}(x, E) \leq d_{1}:=\operatorname{dist}\left(x, x_{1}\right) \leq \operatorname{dist}(x, E)+\varepsilon .
$$

Take any $\xi \in S_{x}^{*} M$ and define the geodesic path $\gamma(t)=\pi \circ \varphi_{t}((x, \xi))$ for $t \in\left[0, d_{1}\right]$. According to Lemma B.1, we have

$$
\text { length }\left(\pi \circ \varphi_{t}((x, \xi))_{\mid[0, T]}\right)=T, \quad \text { for all } T>0 .
$$

Hence, we have $\gamma(t) \notin E$ for $t \in\left[0, d_{1}-\varepsilon\right]$, otherwise we would have $\operatorname{dist}(x, E) \leq d_{1}-\varepsilon$, which contradicts (B.2). The same arguments proves that if we define $\widetilde{\gamma}(t)=\pi \circ \varphi_{t}((x,-\xi))$ defined on $\left[0, d_{1}-\varepsilon\right]$, we have $\widetilde{\gamma}(t) \notin E$ for $t \in\left[0, d_{1}-\varepsilon\right]$. Using Lemma B.2, we also have $\widetilde{\gamma}(t)=\pi \circ \varphi_{-t}((x, \xi))$ on $\left[0, d_{1}-\varepsilon\right]$.

The curve $t \mapsto \pi \circ \varphi_{t}((x, \xi))$ for $t \in\left[-d_{1}+\varepsilon, d_{1}-\varepsilon\right]$ is thus the concatenation of the two geodesics $\gamma$ and $\widetilde{\gamma}$. This is still a geodesic of length $2 d_{1}-2 \varepsilon$ that does not intersect $E$. Therefore, we have $T_{G C C}(E) \geq 2 d_{1}-2 \varepsilon$. The first part of (B.2) gives $T_{G C C}(E) \geq 2 \operatorname{dist}(x, E)-2 \varepsilon$. This gives the result.

Remark B.5. In the case $\partial M \neq \emptyset$, we also have $T_{U C}(\omega) \leq T_{G C C}(\omega)$ for $\omega$ open subsets of $M$, as well as $T_{U C}(\Gamma) \leq T_{G C C}(\Gamma)$ for $\Gamma$ open subsets of $\partial M$. The proof is similar, replacing $\varphi_{t}$ by the appropriate broken bicharacteristic flow (see [31] or [18], Chap. XXIV).

In what follows, we assume $\partial M=\emptyset$. 
Lemma B.6 (Equality case). Assume $T_{G C C}(E)=2 \mathcal{L}(M, E)=2 R_{0}>0$, then, there is $x_{0} \in M$ such that $\operatorname{dist}\left(x_{0}, E\right)=R_{0}$ and for every $\xi \in S_{x_{0}}^{*} M$

$$
\begin{array}{ll}
\pi \circ \varphi_{t}\left(\left(x_{0}, \xi\right)\right) \notin E & \forall|t|<R_{0} \\
\pi \circ \varphi_{t}\left(\left(x_{0}, \xi\right)\right) \in \bar{E} & \forall|t|=R_{0} .
\end{array}
$$

Moreover, these properties are also satisfied by any $x_{0} \in M$ such that $\operatorname{dist}\left(x_{0}, E\right)=R_{0}$.

Finally, for any $x \in M$, we have the following alternative:

- either $\operatorname{dist}(x, E)<R_{0}$,

- or $\operatorname{dist}(x, E)=R_{0}$ and the connected component of $M \backslash \bar{E}$ containing $x$ is the open ball $B\left(x, R_{0}\right)$.

Proof. The function $x \mapsto \operatorname{dist}(x, E)$ is a continuous function on the compact manifold $M$. Consider $x_{0}$ one of the points where it takes its maximum $R_{0}=\operatorname{dist}\left(x_{0}, E\right)=\mathcal{L}(M, E)$.

For any $\xi \in S_{x_{0}}^{*} M$, we have necessarily $\pi \circ \varphi_{t}\left(\left(x_{0}, \xi\right)\right) \notin E \forall|t|<R_{0}$, otherwise, we would have $\operatorname{dist}\left(x_{0}, E\right)<R_{0}$.

Moreover, assume that there exists $\xi_{0} \in S_{x_{0}}^{*} M$ so that $\pi \circ \varphi_{R_{0}}\left(\left(x_{0}, \xi_{0}\right)\right) \notin \bar{E}$. By continuity of $t \mapsto \pi \circ$ $\varphi_{t}\left(\left(x_{0}, \xi_{0}\right)\right)$ and the fact that the complementary of $\bar{E}$ is open, there exists $\varepsilon>0$ so that $\pi \circ \varphi_{t}\left(\left(x_{0}, \xi_{0}\right)\right) \notin \bar{E}$ for $t \in] R_{0}-\varepsilon, R_{0}+\varepsilon\left[\right.$. In particular, by combining with the previous result, we have that $\pi \circ \varphi_{t}\left(\left(x_{0}, \xi_{0}\right)\right) \notin E$ for $t \in]-R_{0}, R_{0}+\varepsilon\left[\right.$. We have constructed a geodesic path of length at least $2 R_{0}+\varepsilon / 2$ that does not intersect $E$. This implies, in particular, that $T_{G C C}(E) \geq 2 R_{0}+\varepsilon / 2$, which is a contradiction.

We now prove the last statement. According to the definition of $R_{0}=\mathcal{L}(M, E)$, the situation $\operatorname{dist}(x, E)>R_{0}$ does not happen, so that we only have to consider points $x_{0}$ with $\operatorname{dist}\left(x_{0}, E\right)=R_{0}$. For such a point $x_{0}$, let $U$ be the connected component of $M \backslash \bar{E}$ containing $x_{0}$. We first prove that $U \subset B\left(x_{0}, R_{0}\right)$. The set $M \backslash \bar{E}$ is open so that $U$ is connected and open, an hence pathwise connected. Given $x \in U$, there exists $\gamma:[0,1] \rightarrow U \subset M \backslash \bar{E}$ a continuous path so that $\gamma(0)=x_{0}$ and $\gamma(1)=x$. In particular, $\gamma(t) \notin \bar{E}$ for $t \in[0,1]$. Assume $\operatorname{dist}\left(x_{0}, x\right) \geq R_{0}$. By continuity, there exists $t_{0} \in[0,1]$ so that $\operatorname{dist}\left(x_{0}, \gamma\left(t_{0}\right)\right)=R_{0}$. There is a geodesic miminizing the distance between $\gamma\left(t_{0}\right)$ and $x_{0}$. That is, there exists $\xi_{0} \in S_{x_{0}}^{*} M$ so that $\pi \circ \varphi_{R_{0}}\left(\left(x_{0}, \xi_{0}\right)\right)=\gamma\left(t_{0}\right)$. In particular, by the previous statement, $\gamma\left(t_{0}\right) \in \bar{E}$. This is a contradiction. So, we have proved $U \subset B\left(x_{0}, R_{0}\right)$. Now, we check that $\operatorname{dist}\left(x_{0}, E\right)=R_{0}$ implies $B\left(x_{0}, R_{0}\right) \subset M \backslash \bar{E}$. Indeed, let $x \in M$ with $\operatorname{dist}\left(x_{0}, x\right)<R_{0}$. Then, $x \notin \bar{E}$ since otherwise we would have $\operatorname{dist}\left(x_{0}, \bar{E}\right)<R_{0}$ which contradicts $\operatorname{dist}\left(x_{0}, \bar{E}\right)=\operatorname{dist}\left(x_{0}, E\right)=R_{0}$. So $B\left(x_{0}, R_{0}\right) \subset M \backslash \bar{E}$. But since $B\left(x_{0}, R_{0}\right)$ is connected and contains $x_{0}$, we have $B\left(x_{0}, R_{0}\right) \subset U$ by definition of $U$. This gives finally $B\left(x_{0}, R_{0}\right)=U$.

Remark B.7. Note that we have the two equivalences $T_{G C C}(E)=0 \Longleftrightarrow(E$ satisfies GCC and $\bar{E}=M)$, and $T_{U C}(E)=0 \Longleftrightarrow \bar{E}=M$.

The following result is used in Section 2.3.

Lemma B.8. Let $\omega$ be an open subset of $M$ satisfying $G C C$ and such that $T_{U C}(\omega)<T_{G C C}(\omega)$. Then, there exists an open subset $\omega_{0}$ of $M$ such that

$$
\bar{\omega}_{0} \subset \omega, \text { and } T_{U C}\left(\omega_{0}\right)<T_{G C C}(\omega) .
$$

Proof. We prove the more general fact for an open set $\omega \subset M$ :

For any $\varepsilon>0$, there exists an open set $\omega_{0}$ with $\bar{\omega}_{0} \subset \omega$ so that $\mathcal{L}\left(M, \omega_{0}\right) \leq \mathcal{L}(M, \omega)+\varepsilon$.

By compactness of $M$, we can find a finite family of points $\left(x_{i}\right)_{i \in I}, x_{i} \in M$, with $I$ finite, so that

$$
M=\cup_{i \in I} B\left(x_{i}, \varepsilon / 2\right),
$$


where $B(y, r)=\{x \in M$, dist $(x, y)<r\}$. By definition of $\mathcal{L}(M, \omega)$, for any $x_{i}$, we have $\operatorname{dist}\left(x_{i}, \omega\right) \leq \mathcal{L}(M, \omega)$ and there exists $y_{i} \in \omega$ so that $\operatorname{dist}\left(x_{i}, y_{i}\right) \leq \mathcal{L}(M, \omega)+\varepsilon / 2$.

Now, since $\omega$ is an open set, there exists $r_{i}>0$ so that $\overline{B\left(y_{i}, r_{i}\right)} \subset \omega$. Now, we take

$$
\omega_{0}:=\cup_{i \in I} B\left(y_{i}, r_{i}\right) .
$$

For any $x \in M$, we can pick $i \in I$ so that $x \in B\left(x_{i}, \varepsilon / 2\right)$. In particular, $\operatorname{dist}\left(x, y_{i}\right) \leq \operatorname{dist}\left(x, x_{i}\right)+\operatorname{dist}\left(x_{i}, y_{i}\right) \leq$ $\mathcal{L}(M, \omega)+\varepsilon$. Therefore, for any $x \in M$, we have $\operatorname{dist}\left(x, \omega_{0}\right) \leq \mathcal{L}(M, \omega)+\varepsilon$. This gives $\mathcal{L}\left(M, \omega_{0}\right) \leq \mathcal{L}(M, \omega)+\varepsilon$. That $\bar{\omega}_{0} \subset \omega$ comes from $\overline{B\left(y_{i}, r_{i}\right)} \subset \omega$ for all $i \in I$ and the finiteness of $I$. This concludes the proof of (B.3), and hence of the lemma.

Acknowledgements. The authors would like to thank Kim-Dang Phung for very interesting conversations about quantitative unique continuation estimates, which were crucial at a very early stage of this paper.

\section{REFERENCES}

[1] C. Bardos, G. Lebeau and J. Rauch, Un exemple d'utilisation des notions de propagation pour le contrôle et la stabilisation de problèmes hyperboliques. Nonlinear hyperbolic equations in applied sciences. Rend. Sem. Mat. Univ. Politec. Torino (Special Issue) (1989) 11-31.

[2] C. Bardos, G. Lebeau and J. Rauch, Sharp sufficient conditions for the observation, control, and stabilization of waves from the boundary. SIAM J. Contr. Opt. 30 (1992) 1024-1065.

[3] L. Baudouin, M. De Buhan and S. Ervedoza, Global Carleman estimates for waves and applications. Comm. Partial Differ. Eq. 38 (2013) 823-859.

[4] R. Bosi, Y. Kurylev and M. Lassas, Stability of the unique continuation for the wave operator via tataru inequality and applications. Preprint arXiv:1506.04318 (2015).

[5] N. Burq and P. Gérard, Condition nécessaire et suffisante pour la contrôlabilité exacte des ondes. C. R. Acad. Sci. Paris Sér. I Math. 325 (1997) 749-752.

[6] F. Chaves-Silva and G. Lebeau, Announcement (2015).

[7] J.-M. Coron and S. Guerrero, Singular optimal control: A linear 1-D parabolic-hyperbolic example. Asympt. Anal. 44 (2005) 237-257.

[8] J.-M. Coron, Control and nonlinearity. Vol. 136 of Mathematical Surveys and Monographs. American Mathematical Society, Providence, RI (2007).

[9] B. Dehman and S. Ervedoza, Dependence of high-frequency waves with respect to potentials. SIAM J. Control Optim. 52 (2014) 3722-3750.

[10] B. Dehman and G. Lebeau, Analysis of the HUM control operator and exact controllability for semilinear waves in uniform time. SIAM J. Control Optim. 48 (2009) 521-550.

[11] B. Dehman, J. Le Rousseau and M. Léautaud, Controllability of two coupled wave equations on a compact manifold. Arch. Ration. Mech. Anal. 211 (2014) 113-187.

[12] S. Dolecki and D.L. Russell, A general theory of observation and control. SIAM J. Control Optim. 15 (1977) 185-220.

[13] T. Duyckaerts, X. Zhang and E. Zuazua, On the optimality of the observability inequalities for parabolic and hyperbolic systems with potentials. Ann. Inst. Henri Poincaré, Anal. Non Linéaire 25 (2008) 1-41.

[14] S. Ervedoza and E. Zuazua, A systematic method for building smooth controls for smooth data. Discrete Contin. Dyn. Syst. Ser. B 14 (2010) 1375-1401.

[15] S. Gallot, D. Hulin and J. Lafontaine, Riemannian geometry. Universitext, 3rd edn. Springer-Verlag, Berlin (2004).

[16] P. Gérard, Microlocal defect measures. Comm. Partial Differ. Eq. 16 (1991) 1761-1794.

[17] A. Haraux, T. Liard and Y. Privat, How to estimate observability constants of one-dimensional wave equations? propagation versus spectral methods. J. Evol. Equ. (2016) 1-32.

[18] L. Hörmander, The Analysis of Linear Partial Differential Operators, Volume III. Springer-Verlag (1985). Second printing (1994).

[19] L. Hörmander, A uniqueness theorem for second order hyperbolic differential equations. Commun. Partial Differ. Eq. 17 (1992) 699-714.

[20] L. Hörmander, On the uniqueness of the Cauchy problem under partial analyticity assumptions. In Geometrical optics and related topics (Cortona, 1996). Vol. 32 of Progr. Nonlin. Differ. Eq. Appl. Birkhäuser Boston, Boston, MA (1997) 179-219.

[21] F. John, On linear partial differential equations with analytic coefficients. Unique continuation of data. Comm. Pure Appl. Math. 2 (1949) 209-253.

[22] V. Komornik, Exact controllability and stabilization. RAM: Res. Appl. Math. Masson, Paris (1994). The multiplier method. 
[23] C. Laurent and M. Léautaud, Quantitative unique continuation for operators with partially analytic coefficients. Application to approximate control for waves. Preprint arXiv:1506.04254 (2015).

[24] G. Lebeau, Contrôle analytique. I. Estimations a priori. Duke Math. J. 68 (1992) 1-30.

[25] G. Lebeau, Équation des ondes amorties. In Algebraic and geometric methods in mathematical physics (Kaciveli, 1993). Vol. 19 of Math. Phys. Stud. Kluwer Acad. Publ., Dordrecht (1996) 73-109.

[26] N. Lerner, Uniqueness for an ill-posed problem. J. Differ. Eq. 71 (1988) 255-260.

[27] N. Lerner, Metrics on the phase space and non-selfadjoint pseudo-differential operators. Birkhäuser-Verlag, Basel (2010).

[28] J.-L. Lions, Contrôlabilité exacte, perturbations et stabilisation de systèmes distribués. Tome 1, Vol. 8 of Recherches en Mathématiques Appliquées. Masson, Paris (1988).

[29] P. Lissy, A link between the cost of fast controls for the 1-D heat equation and the uniform controllability of a 1-D transport-diffusion equation. C. R. Math. Acad. Sci. Paris 350 (2012) 591-595.

[30] P. Lissy, Explicit lower bounds for the cost of fast controls for some 1-D parabolic or dispersive equations, and a new lower bound concerning the uniform controllability of the 1-D transport-diffusion equation. J. Differ. Eq. 259 (2015) $5331-5352$.

[31] R.B. Melrose and J. Sjöstrand, Singularities of boundary value problems. I. Comm. Pure Appl. Math. 31 (1978) $593-617$.

[32] L. Miller, Escape function conditions for the observation, control, and stabilization of the wave equation. SIAM J. Control Optim. 41 (2002) 1554-1566.

[33] L. Miller, Geometric bounds on the growth rate of null-controllability cost for the heat equation in small time. J. Differ. Eq. 204 (2004) 202-226.

[34] L. Miller, How violent are fast controls for Schrödinger and plate vibrations? Arch. Ration. Mech. Anal. 172 (2004) 429-456.

[35] L. Miller, Controllability cost of conservative systems: resolvent condition and transmutation. J. Funct. Anal. 218 (2005) 425-444.

[36] K.D. Phung, Waves, damped wave and observation. In Some problems on nonlinear hyperbolic equations and applications. Vol. 15 of Ser. Contemp. Appl. Math. CAM. Higher Ed. Press (2010) 386-412.

[37] J. Rauch and M. Taylor, Penetrations into shadow regions and unique continuation properties in hyperbolic mixed problems. Indiana Univ. Math. J. 22 (1972/73) 277-285.

[38] J. Rauch and M. Taylor, Exponential decay of solutions to hyperbolic equations in bounded domains. Indiana Univ. Math. J. 24 (1974) 79-86.

[39] L. Robbiano, Théorème d'unicité adapté au contrôle des solutions des problèmes hyperboliques. Commun. Partial Differ. Eq. 16 (1991) 789-800.

[40] L. Robbiano, Fonction de coût et contrôle des solutions des équations hyperboliques. Asymptotic Anal. 10 (1995) $95-115$.

[41] J. Royer, Analyse haute fréquence de l'équation de Helmholtz dissipative. Thèse de Doctorat. Université de Nantes (2010). Available at http://www.math.sciences.univ-nantes.fr/ jroyer/these.pdf.

[42] L. Robbiano and C. Zuily, Uniqueness in the Cauchy problem for operators with partially holomorphic coefficients. Invent. Math. 131 (1998) 493-539.

[43] J. Royer, Limiting absorption principle for the dissipative Helmholtz equation. Commun. Partial Differ. Eq. 35 (2010) $1458-1489$.

[44] R.T. Seeley, Complex powers of an elliptic operator. In Singular Integrals (Proc. of Sympos. Pure Math., Chicago, Ill., 1966). Amer. Math. Soc., Providence, R.I. (1967) 288-307.

[45] T.I. Seidman, How violent are fast controls? Math. Control Signals Systems 1 (1988) 89-95.

[46] M.A. Shubin, Pseudodifferential Operators and Spectral Theory, 2nd edn. Springer-Verlag, Berlin Heidelberg (2001).

[47] L. Tartar, $H$-measures, a new approach for studying homogenisation, oscillations and concentration effects in partial differential equations. Proc. Roy. Soc. Edinburgh Sect. A 115 (1990) 193-230.

[48] D. Tataru, Unique continuation for solutions to PDE's; between Hörmander's theorem and Holmgren's theorem. Commun. Partial Differ. Eq. 20 (1995) 855-884.

[49] D. Tataru, Carleman estimates, unique continuation and applications. Lecture notes. Available at https://math. berkeley.edu/ tataru/papers/ucpnotes.ps (1999).

[50] D. Tataru, Unique continuation for operators with partially analytic coefficients. J. Math. Pures Appl. 78 (1999) 505521.

[51] M.E. Taylor, Reflection of singularities of solutions to systems of differential equations. Comm. Pure Appl. Math. 28 (1975) 457-478.

[52] M.E. Taylor, Partial differential equations II. Qualitative studies of linear equations, Vol. 116 of Applied Mathematical Sciences, 2nd edn. Springer, New York (2011).

[53] E. Zuazua, Exact controllability for semilinear wave equations in one space dimension. Ann. Inst. Henri Poincaré, Anal. Non Lin. 10 (1993) 109-129. 